\title{
Personalised medicine and drug development
}

\author{
Citation for published version (APA):
}

Leyens, L. (2017). Personalised medicine and drug development: Analysis of the current landscape, flexible regulatory pathways and decision supporting tools. [Doctoral Thesis, Maastricht University]. Datawyse / Universitaire Pers Maastricht. https://doi.org/10.26481/dis.20171011/l

Document status and date:

Published: 01/01/2017

DOI:

10.26481/dis.20171011\|

Document Version:

Publisher's PDF, also known as Version of record

\section{Please check the document version of this publication:}

- A submitted manuscript is the version of the article upon submission and before peer-review. There can be important differences between the submitted version and the official published version of record.

People interested in the research are advised to contact the author for the final version of the publication, or visit the DOI to the publisher's website.

- The final author version and the galley proof are versions of the publication after peer review.

- The final published version features the final layout of the paper including the volume, issue and page numbers.

Link to publication

\footnotetext{
General rights rights.

- You may freely distribute the URL identifying the publication in the public portal. please follow below link for the End User Agreement:

www.umlib.nl/taverne-license

Take down policy

If you believe that this document breaches copyright please contact us at:

repository@maastrichtuniversity.nl

providing details and we will investigate your claim.
}

Copyright and moral rights for the publications made accessible in the public portal are retained by the authors and/or other copyright owners and it is a condition of accessing publications that users recognise and abide by the legal requirements associated with these

- Users may download and print one copy of any publication from the public portal for the purpose of private study or research.

- You may not further distribute the material or use it for any profit-making activity or commercial gain

If the publication is distributed under the terms of Article $25 \mathrm{fa}$ of the Dutch Copyright Act, indicated by the "Taverne" license above, 
Personalised Medicine and drug development: analysis of the current landscape, flexible regulatory pathways and decision supporting tools 
(C) copyright Author Lada Leyens, Maastricht 2017

Printing: Datawyse | Universitaire Pers Maastricht

ISBN 9789461597571

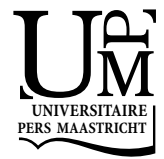




\title{
Personalised Medicine and drug development:
}

\author{
analysis of the current landscape, \\ flexible regulatory pathways and decision \\ supporting tools
}

\author{
DISSERTATION \\ To obtain the degree of Doctor at the Maastricht University on the authority of the \\ Rector Magnificus, \\ Prof. dr. Rianne M. Letschert \\ in accordance with the decision of the Board of Deans, \\ to be defended in public on the $11^{\text {th }}$ October, 2017 at 14:00 \\ by \\ Lada Leyens
}

born July 28th 1987 in Palma de Mallorca, Spain 


\section{Supervisor:}

Prof.dr. A. Brand

\section{Co-Supervisor:}

Dr. N. Malats, Spanish National Cancer Research Centre, CNIO, Spain

\section{Assessment Committee:}

Prof.dr. L. Curfs (Chair)

Prof.dr. E. Anklam, Joint Research Centre, European Commission, Belgium Prof.dr.med. M. Papaluca, European Medicines Agency, United Kingdom Prof.dr. S. Ramani

Dr. P. Schröder-Bäck 
"If you want to build a ship, don't herd people together to collect wood and don't assign them tasks and work, but rather teach them to long for the endless immensity of the sea"

Antoine de Saint-Exupery 



\section{Contents}

$\begin{array}{ll}\text { Abbreviations } & 9\end{array}$

$\begin{array}{lll}\text { Chapter } 1 \text { Introduction } & 11\end{array}$

Chapter 2 Working towards personalization in medicine: main obstacles to reaching this vision from today's perspective

Chapter 3 An Index of Barriers for the Implementation of Personalised Medicine and Pharmacogenomics in Europe

Chapter 4 Available tools to facilitate early patient access to medicines in EU and the USA: analysis of conditional approvals and the implications for personalized medicines

Chapter 5 Early patient access to medicines: Health Technology Assessment Bodies need to catch up with new Marketing Authorization methods

Chapter 6 Use of Big Data for drug development and for Public and Personal Health and Care

Chapter 7 Tools from other scientific fields: game theory

Chapter 8 General discussion

Curriculum Vitae

Valorisation

Summary

Acknowledgements

Annex 1 CSA PerMed: Europe’s commitment to Personalised Medicine 



\section{Abbreviations}

CA Competent authorities

AP Adaptive pathways

CDx Companion diagnostic

CHMP Committee for Medicinal Products for Human Use

CMA Conditional marketing authorization

EC Ethic committees

EHTAA European Health Technology Assessment Agency (hypothetical name)

EMA European Medicines Agency

EMREA European Medicines Relative Effectiveness Agency (hypothetical name)

EU European Union

FDA Food and Drug Administration (USA)

FIM First-in-man clinical trial

GCP Good Clinical Practice

GLP Good Laboratory Practice

GMP Good Manufacturing Practice

GVP Good Pharmacovigilance Practice

HCPs Healthcare professionals

HTA Health Technology Assessment

HTAB Health Technology Assessment Body

ICH International conference on harmonization

ICT Information and Communication Technologies

IRB Institutional review boards

JA Joint action

MAA Marketing Authorization Application procedure

MAPPs Medicines adaptive pathways to patients

MEA Managed Entry Agreements

MSs Member states

MTD Maximum tolerated dose

P\&R Pricing and reimbursement

PAM post-authorization measures 
PM Personalised Medicine

PRIME Priority medicines scheme

QALY Quality Adjusted Life Years

R/B Risk / benefit

RCT Randomised controlled clinical trial

REA Relative Effectiveness assessment

RWD / Real-world data / real-world evidence

RWE

SME

Small and medium enterprise

SRIA Strategic Research and Innovation Agenda

SWOT Strengths, weaknesses, opportunities and threats 


\section{Chapter 1}

\section{Introduction}

"(..) far more important to know what person the disease has than what disease the person has"

Hippocrates (c. 460-370 BCE) 



\subsection{Personalised Medicine}

The past decade has seen a paradigm shift in medicine, moving from the anatomical and histological classification of diseases, to the molecular classification we see nowadays. "Static" biomarkers are being used more and more as diagnostic tools, which break down conditions previously considered as one into separate diseases with different pathways (Munoz \& Kurzrock, 2012). More targeted medicines are also being developed for small specific populations based on these static biomarkers. As a result, we are experiencing the rise of the "personalised medicine" era, which will continue developing, even more so as "dynamic" biomarkers (such as the epigenome, the changing environment, the microbiome, tumour heterogeneity, etc.) are introduced in the diagnosis and treatment of diseases.

Personalised treatments options are being developed for an array of different conditions and some have already entered the market. Oncology is leading the field, but they are followed closely by treatments in cardiovascular, pulmonary and psychiatric conditions, between others. Personalised therapeutics aim to provide truly individualised treatments to patients; their societal value lies in the increased efficacy, reduced number of adverse events, reduced uncertainty about favourable outcomes and increased adoption and compliance, resulting in cost savings and reduction of general morbidity in the population (see Box 1 for definitions).

Box 1. Definitions of personalised medicine from Europe and USA. The European definition (first) is the working definition for this thesis.

"Personalised Medicine refers to a medical model using characterisation of individuals' phenotypes and genotypes (e.g. molecular profiling, medical imaging) for tailoring the right therapeutic strategy for the right person at the right time, and/or to determine the predisposition to disease and/or to deliver timely and targeted prevention". (CSA PerMed, 2015)

"Personalized Medicine is the tailoring of medical treatment to the individual characteristics of each patient. The approach relies on scientific breakthroughs in our understanding of how a person's unique molecular and genetic profile makes them susceptible to certain diseases. This same research is increasing our ability to predict which medical treatments will be safe and effective for each patient, and which ones will not be." (Personalized Medicine Coalition, 2011) 
Despite being a concept that is constantly applied by physicians and was already used more than two thousand years ago in ancient Greece, the advances in the socalled "-osmic" sciences (genomics, transcriptomics, proteomics, metabolomics, microbiomics, etc.) and in Information and Communication Technologies (ICT) have led to enormous progress in the field of personalised medicine in the past two decades. The greater understanding of the molecular basis of disease and all the factors influencing disease onset, progression and response to treatment alone could not result in the developments we have experienced. It could only come as a result of the staggering fall in the costs of gene sequencing and genotyping, the increased speed of results availability and the improvement of analytical and interpretation methods. Furthermore, the increased interest from physicians, decision makers, regulators and the general public on personalised medicine has also contributed to its application.

\section{Some history}

The approval of Roche/Genentech's Herceptin ${ }^{\circledR}$ (trastuzumab), for the treatment of HER2+ breast cancer in combination with its companion diagnostic (CDx) for HER2 protein in 1998 by the USA Food and Drug Administration (FDA) and subsequently by the European Medicines Agency (EMA) marked the beginning of an era. Thereafter, about two-dozen treatments have been approved with the required use of a CDx to identify the right population, and around two dozens more with recommended use of a CDx or as information only (Personalized Medicine Coalition, 2016). However, the translation of personalised therapeutics into the market has not been as successful and quick as expected in the late 90s after Herceptin ${ }^{\circledR}$ obtained the marketing authorization (Owens \& Breithaupt, 2002).

Europe and the USA are showing a clear commitment to personalised medicine. The European Commission has published reports supporting the mission of personalisation of health (European Commission, 2013). Many politicians and decision makers have expressed their support and the Horizon2020 research grant program -initiated in January 2014- promotes research in all aspects of targeted therapies, including ICT to assist decision making in personalised medicine. The cornerstones of the European commitment are: IC PerMed, CSA PerMed (both described in detail below) and the Luxembourg Presidency Council Conclusions on personalised medicine (Council of the European Union, 2015). Furthermore, national governments such as the British and French have made implicit or explicit commitments to genomic medicine and the application of personalised medicine, mostly in cancer research and treatment. 


\section{It is not all about genetics and cancer}

But is it all about genetics and cancer? Indeed not, we need to see beyond the concept that personalised medicine is exclusively about the genome. The new insights in systems biology depict a complex matrix of interactions between many factors that may lead to disease. The genome, the environment, life-style, nutrition, epigenome, microbiome, etc., they all play a role in disease onset, progression and response to treatment. The information obtained from the genome at a certain time-point is only a snapshot of that exact moment; we are dealing with dynamic information that changes over the life-course of an individual and that needs to be monitored regularly for changes (Brand, 2009).

There are some interesting new developments with in silico models that, for example, predict how a patient may respond to an array of drugs by studying patient-derived cancer cells a priori (Pingle, et al., 2014), or to simulate virtual clinical trials where virtual patients (modelled from real-life data) are treated with different drugs virtually (Lehrach, 2015). Approaches like this one, using validated in-silico models, could be useful for designing the therapy of individual patients and for informed treatment decision making. Patient-specific multi-scale models are also being developed in the field of nutrigenomics to predict metabolic and inflammatory processes in the onset and progression of Type 2 Diabetes; this model uses genetic, metabolic, and nutritional data for risk-prediction, diagnosis, clinical decision making and therapy response prediction (Castiglione, Tieri, Ernst, \& et al., 2013). The applications of nutrigenomics are also being explored for atherosclerosis, inflammatory bowel disease and cancer between others. In Hepatitis C, a simple model that predicts necessary treatment duration based on the time to initial viral response was tested with promising results that could lead to significant cost savings and reduction of safety risks (Francioso, Almerighi, Forte, Bandiera, Angelico, \& et al., 2014). These are only some examples of the predictive models that combine various information sets to forecast the best treatment strategy for individual patients; however, the potential and the applicability are much larger than this.

\section{Benefits and challenges}

As already mentioned, there are vast benefits from applying evidence-based personalised treatments. Nevertheless, a lot has to be achieved in order to reach these benefits across the entire healthcare spectrum, and not restrict them to a limited number of conditions and citizens. The challenges have been widely discussed and described in a large number of reports and publications, which regardless of their authors, interests and target public, reach similar conclusions on the aspects that need to be tackled (see chapter 2). 
Targeted research to better understand the molecular mechanisms of disease and all implicated factors, as well as the identification of adequate biomarkers, is essential for the development of further personalised therapeutics. Multidisciplinary research teams, pooling knowledge from various disciplines, as well as cross-disciplinary and cross-border collaboration in research and in drug development are essential for the development of personalised medicines. Further improvements in data collection, storage, management, sharing, mining, processing and analysing are also imperative. ICT have not been exploited to their full potential and will surely push forward the individualization of medicine in all areas (research, translation, diagnosis, treatment decision making, follow-up, etc.) (see chapter 6).

Changes in the current business model for pharmaceutical companies are also needed: pre-competitive collaboration between companies (pharmaceutical companies and medical device manufacturers for example), the increase in publicprivate partnerships and a more flexible and adaptive business model are essential for the development and translation of personalised technologies into healthcare. Furthermore, early dialogue with regulators at an early phase of development would lead to more efficient drug development and translation processes (see chapters 4 and 5). Clinical Trial designs also need to adapt to these new treatment approaches. Phase III studies with large number of patients are not possible in small disease subsets and adaptive clinical trial designs with smaller populations are needed (as already applied in some areas of oncology, see section 1.3 below).

In addition, the regulations that are in place nowadays do not always consider the specificities of personalised therapeutics. Many of the ones that affect personalised medicine (Clinical Trial Directive, Data Protection Directive, Medical Device Directive, etc.) have been recently revised and their effect needs to be evaluated. Some positive steps forward are the recently finalised medical devices regulation regulating for the first time in Europe companion diagnostics- and the proposed flexible regulatory approaches from the EMA (see chapter 4). Nonetheless, these and similar efforts are in vain in Europe if Health Technology Assessment (HTA) bodies and payers do not provide flexible pricing and reimbursement (P\&R) methods to ensure timely patient access to promising new medicines (see chapter 5).

When it comes to healthcare systems, a general change in mind-set in healthcare delivery and provision is needed. From a coordinated reimbursement process for drugs and diagnostics, new financing strategies and new structures and models at the provider level to an update in healthcare professional training. Furthermore, a change in patient behaviour, increased interest and literacy from citizens and in general a change in the sector's mentality and attitude are needed to facilitate a shift towards Predictive, Preventive, Personalised and Participatory healthcare (see chapter 3 ). To achieve this, all stakeholders need to apply the principles of 
ELSI $^{1}$ to ensure that the most ethical approach is taken, since the social consequences of the full implementation of personalised medicine have not been fully analysed and many ethical challenges lay ahead.

New dynamic and sustainable pathways are needed to allow the timely and effective translation of innovative technologies into healthcare and health polices; always ensuring high quality, safe and effective treatments enter the market, and well-functioning models and programs are used to decide treatment options.

\subsection{Medicines development: from the lab to the patient}

Chapters 4 and 5 discuss the drug approval process and assume a basic knowledge of the different stages in the drug development process. Below a high-level summary of this process is presented as introduction to these chapters.

\section{Drug development}

The process of drug development starts in the lab. Scientists investigate the biological pathways of disease and look for drugs and molecules that target these pathways and cure or treat the condition. The previously mentioned paradigm shift in diagnosis and treatment is leading to a change in the drug development process. What used to be a process to develop medicines to treat many patients with the same compound (the so called "one-size-fits-all" blockbuster drugs, such as Rofecoxib -Vioxx- and atorvastatin -Lipitor) and took 1000 medicines to be investigated in the lab for one successful candidate to reach the patient; is becoming a more targeted development process. In this targeted process, a small number of similar compounds are tested to find the correct one that will reach the patient. The improvement in development and manufacturing techniques as well as the latest advances in biological research and ICT (e.g. in silico models) are yielding highly innovative and targeted medicines, many of which can be considered personalised medicines.

A compound must pass numerous tests that ensure its safety and efficacy before it reaches the patient. Firstly, it is tested ex-vivo in the lab using cells, it then goes through an array of in vivo tests in animals (pre-clinical tests) to ensure it is as safe as possible for the first in human clinical trial and to do preliminary proof of concept efficacy tests. These pre-clinical tests are defined in the $\mathrm{ICH}^{2}$ safety guidelines. The animal species used for these tests depend on the condition, the type of compound and the appropriate animal model for the disease to treat. They

\footnotetext{
${ }^{1}$ ELSI: Ethical, Legal and Social Implications

${ }^{2}$ International Conference on Harmonization guidelines endorsed by the major World countries and regions. http://www.ich.org/products/guidelines
} 
are mostly rodents, rabbits (usually over-sensitive), canines and sometimes primates (although tests in primates are not allowed in some countries). The drug's safety is tested through carcinogenicity, genotoxicity, toxicokinetics, toxicity, reproductive toxicity, immunotoxicology and in some cases photosafety studies (ICH, 2016). The effects of the drug on the most important organs and body systems (cardiac, respiratory, digestive, etc.) are also tested with standard preclinical tests to predict the possible adverse events in humans. Even though some tests are universal, not all drugs undergo the same studies; biotechnological products and anticancer drugs have their own battery of tests (ICH guidelines S6 and S9 respectively), for example, and other special tests are required for paediatric drugs.

Once the safety in animals, the effect on the different systems and the possible adverse reactions in humans are established it is decided whether the drug is appropriate for human use. If so, the compound is tested for safety in the 'first-inman' clinical trial (FIM) on healthy volunteers or terminal patients (mostly for oncology and other very toxic agents). There are very strict guidelines that must be followed for FIM trials. These guidelines were revised so that drugs are tested in a staged manner to avoid the dramatic events of the 2006 TeGenero scandal in the UK (Trials, 2006); and the second revision is scheduled to be finished by summer 2017 following the 2016 Biotrial accident in France (Touraine, 2016).

\begin{tabular}{|c|c|c|}
\hline $\begin{array}{l}\text { Discovery and pre- } \\
\text { clinical development }\end{array}$ & Clinical development & $\begin{array}{l}\text { Marketing Authorization } \\
\text { and patient access }\end{array}$ \\
\hline $\begin{array}{l}\text { Target identification } \\
\text { Proof of mechanism } \\
\text { ADME } \\
\text { Safety } \\
\text { Toxicology } \\
\text { PK/PD } \\
\text { Dose ranging }\end{array}$ & $\begin{array}{l}\text { Efficacy } \\
\text { Effectiveness } \\
\text { Safety } \\
\text { Human PK } \\
\text { Dose selection } \\
\text { Bioavailability }\end{array}$ & $\begin{array}{l}\text { Ph IV clinical trials } \\
\text { Real-life evidence } \\
\text { Treatment planning and monitoring } \\
\text { Postmarketing surveillance } \\
\text { Diagnosis and prognosis }\end{array}$ \\
\hline
\end{tabular}

Figure 1-1. Current standard drug development process: from the lab to the patient. ADME: absorption, distribution, metabolism, and excretion; PD: Pharmacodynamics; PK: Pharmacokinetics; Ph I / II / III / IV: clinical trial phases; MAA: marketing authorization application; HTA: health technology assessment.

After safety in humans is confirmed in FIM trials and the maximum tolerated dose (MTD) is established, the compound enters phase II testing (see Figure 1-1). Phase II clinical trials evaluate the safety of the compound in a moderate number of patients, they also test the optimal dosage and yield the initial efficacy results. These trials are often designed as randomized controlled studies that test the drug candidate against placebo (if ethically acceptable). Phase I and Phase II trials can 
also be combined into a Phase I/II trial that tests the same variables in a staged manner as they would in two separate protocols. After the optimal dose or doses are established and the safety profile in patients is identified the compound is tested in larger phase III clinical trials.

Phase III trials are designed to evaluate the efficacy of a drug in a given patient population (see Box 2). They are usually randomized controlled multicentre trials (see section 1.3 for the types of clinical trial designs) with large patient populations that provide information on the drug's efficacy, the adverse reactions it causes on patients, how it compares to other available treatments and how to use it safely.

Box 2.Efficacy vs. effectiveness in the clinical trial context

We must distinguish between the efficacy and the effectiveness of a treatment:

Efficacy clinical trials (or explanatory trials) ascertain whether an intervention produces a therapeutic effect as compared to a placebo under ideal circumstances that minimize bias. (Gartlehner, Hansen, Nissman, \& al., 2006)

Effectiveness clinical trials (or pragmatic trials) determine the health benefit of an intervention under routine clinical practice or "real world" settings. Several factors that can affect treatment outcomes are not controlled (Nordon, et al., 2016).

The drug development process explained above is the traditional process that has been followed for many years and is still followed for many compounds. As mentioned above, not all products can go through large Phase III studies. For example, the limited number of patients suffering from orphan diseases is not sufficient to carry out large phase III trials to test orphan drugs. With the rise of personalised medicines we encounter the same scenario as with orphan drugs: conditions that have been usually considered as one are broken down into smaller clusters of diseases with distinctive pathways and small number of patients classified into each one of them. The incidence of these individual clusters is much lower than the incidence of the initial "mother" condition. Drugs that target the pathway of one disease cluster will have a smaller population of patients available for testing than drugs that target the initial "mother" condition. For these personalised therapies large phase III trials are not feasible, and the traditional development process needs to be adapted. As discussed below, and in chapters 4 and 5, new clinical trial designs are emerging for these compounds. Even regulatory agencies are developing new approval pathways and methods, which allow for more flexible development processes and do not prescribe a standard rigid development process that is the same for all drugs. 


\section{Regulatory process}

The regulatory process, in European countries and most other regions, starts with the ethic applications for pre-clinical trials and the application process for clinical trials, which includes applications to the ethic committees (EC -called institutional review boards or IRBs in the USA) and the national regulatory authorities (or competent authorities -CA-). All pre-clinical and clinical trials need to be conducted in accordance to GXP guidelines, which include Good Laboratory Practice -GLP-, Good Manufacturing Practice -GMP-, Good Clinical Practice -GCPand Good Pharmacovigilance Practice -GVP; as well as other ethical guidelines (such as the Declaration of Helsinki), international directives and national directives and regulations. National CAs carry out inspections to ensure these guidelines and regulations are respected.

In Europe, where the landscape for multi-national clinical trials is quite challenging due to differing local interpretations of the European Clinical Trial Directive, the new European Clinical Trial Regulation (Regulation EU No 536/2014) establishes an improved centralised European application process in which each country needs to coordinate the approval process between the EC and the national CA (European Commission, 2014). The regulation will come into force as soon as the European Clinical Trial Portal and Database -currently under development at the EMA- are ready. Until then, the current Clinical Trial Directive (Directive 2001/20/EC) (European Commission, 2001) remains in force.

The process continues with the Marketing Authorization Application procedure (MAA), if the results of clinical trials are positive and the developer (e.g. pharmaceutical company) is confident that the available evidence is sufficient to meet all regulatory requirements for marketing the drug. In Europe, the MAA can be centralised through the EMA or decentralised through the national CAs. For the drugs we are interested in, the centralised procedure is mostly compulsory.

The EMA coordinates the MAA centralised procedure. The Committee for Medicinal Products for Human Use (CHMP) designates one rapporteur and one corapporteur country. The designated national CAs evaluate the evidence presented in the dossier submitted by the applicant and prepare two independent assessment reports within the first 80 days of the review period. All EU countries, plus Norway and Iceland, can comment at any time of the procedure on the reports produced by the rapporteurs, and are involved in the final discussions and decision, which are based on these reports. There are usually two round of questions to the applicant, although the second one is only necessary if there are still open issues that need clarification after the first round of answers. The whole scientific evaluation process takes 210 days (see Figure 1-2); however, the effective time until the CHMP issues an opinion can be considerable longer since the period applicants have to respond are counted as clock-stops which can be 
prolonged. Based on the review process and the final assessment reports, the CHMP issues an opinion on the approvability of the medicine (positive, conditional or negative). Thereafter, the European Commission is the body in charge of issuing the legally binding approval in the form of a marketing authorization automatically valid in all EU member states (MSs) (Schneider \& Schäffner-Dallmann, 2008).

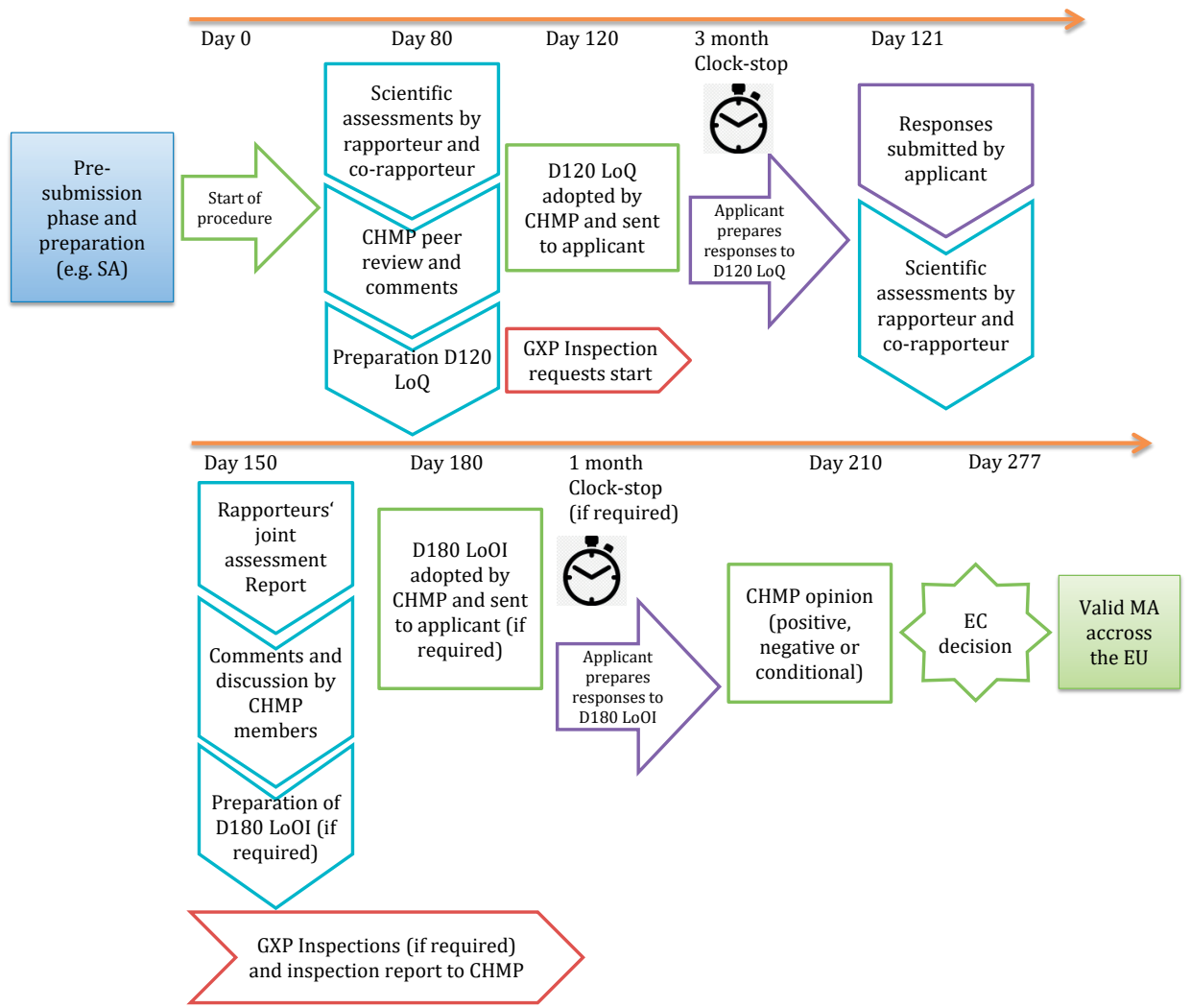

Figure 1-2. Summary of the centralised MAA procedure in Europe, coordinated by the EMA. CHMP: Committee for Medicinal Products for Human Use, D120: day 120, D180: day 180, EC: European Commission, EU: European Union, GXP: Good X Practice, term used to refer to GLP, GMP, GCP, GVP; LoQ: List of Questions, LoOI: List of Outstanding Issues, MA: marketing authorization, SA: scientific advice. Modified from (Schneider \& Schäffner-Dallmann, 2008)

Applicants also have the opportunity to ask questions to the CHMP through the Scientific Advice process, which is open to all applicants at all developmental stages. This can be considered a pre-cursor of the "early dialogue" initiatives (see chapters 4 and 5), although the later are based on the innovative profile of the compound and the unmet need of the population to be treated, and have been conceived to start at the initial stages of development. The answers to the applicant's questions are discussed by the CHMP and a final report is sent back to the applicant with the CHMP's position; this report is not binding at the time of 
MAA. Orphan drugs, small and medium enterprises (SMEs) and drugs intended for paediatric use have some incentives and exemptions on the fees requested for the Scientific Advice procedure.

The above-described process is the standard MAA procedure. As described below (and further discussed in chapters 4 and 8), the EMA, the FDA and other regulatory agencies are developing innovative approval methods and pathways that encourage early dialogue between applicants, regulators and other stakeholders to speed patient access to promising new drugs.

\section{Patient access}

In Europe, the marketing authorization issued by the European Commission is immediately valid in all EU Member States. However, this does not mean that all European patients have access to the authorized medicine immediately after issuing marketing authorization. After the MAA process, medicines go through the reimbursement process. Unfortunately, this process does not follow one single homogeneous procedure in the EU, each country has its own national body (or more than one) and its own procedure, with very different methods, criteria and final decisions (see chapter 5).

HTA Bodies or Payers analyse and determine the value of the medicine in each country separately. Various strategies are used by European countries to control market access such as pricing strategies, reimbursement strategies and limiting access to certain care settings (e.g. in-patient vs. out-patient access, in hospitals vs. ambulatory care). For example, Germany offers at first a "free" pricing system, which is then adjusted depending on the value ${ }^{3}$ of the medicine. France has a value-based pricing system in place, which negotiates the price based on the added clinical benefit. And other countries, such as Italy, use an external referencepricing scheme. Reimbursement strategies are based on selective public financing and often use lists of 'reimbursable' medicines or negative lists of medicines that are not reimbursed in the public healthcare sector; one example of such lists is the NHS positive reimbursement lists (Martinalbo, et al., 2016).

For pricing and reimbursement decisions, HTAs may evaluate the relative effectiveness by looking at the added therapeutic value compared to other marketed options, as is the case in France and Germany. Other bodies/countries may focus on the economic aspect and perform a budget impact analysis; whereas others, such as NICE in England, mix both components and require a costeffectiveness analysis with a price proposed by the company and the final decision

\footnotetext{
${ }^{3}$ Value here refers to added clinical benefit
} 
being based on the cost/QALY ${ }^{4}$ estimate compared to an accepted threshold (between 20,000GBP and 30,000GBP in the case of NICE (NICE, 2015)).

Early access in some countries is available through special schemes and funds, such as the Cancer Drug Fund in the UK, or the various Managed Entry Agreements (MEAs) negotiated individually by each country with pharmaceutical companies. Risk-sharing agreements, payment by result and coverage with evidence development are some of the MEAs negotiated by countries such as France, Italy, Netherlands, Spain or Sweden, in what is often a very un-transparent negotiation process (Ferrario \& Kanavos, 2013).

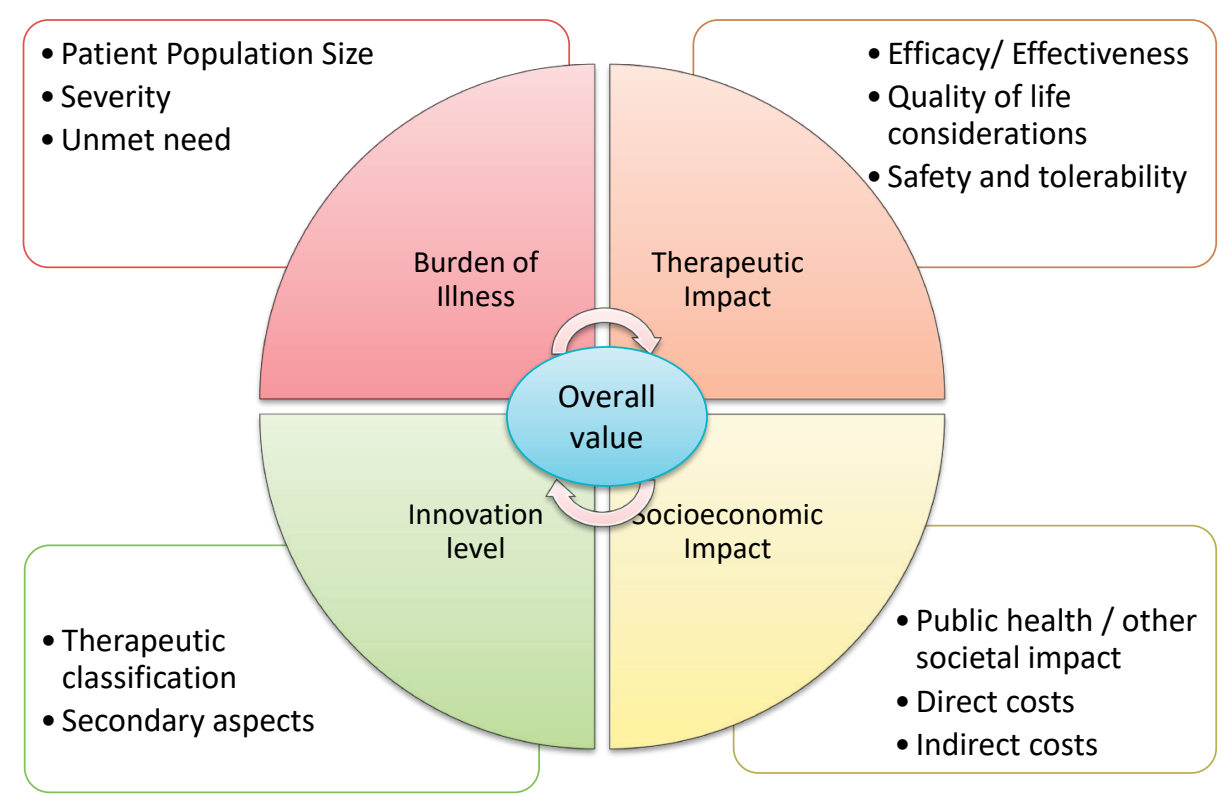

Figure 1-3. The Overall Value equation: important variables to consider when analysing the added therapeutic value (ATV) of medicines. Modified from the ValueVectorTM (Kanavos \& Angelis, 2013)

In order to homogenise the reimbursement process across the EU and avoid a cross-border post-code lottery 5 for European patients, especially regarding the evaluation of relative effectiveness and added clinical benefit (or value), the creation of a European body that coordinates a joint evaluation process (similar to the EMA for the MAA process) has been proposed. Each country would then be free to implement in their territory the final outcome of the Added Therapeutic Value assessment (ATV) since the variables in the value equation (see Figure 1-3) may

\footnotetext{
${ }^{4}$ QALY: Quality Adjusted Life Years. Measure used in health economic analysis.

5 The term "post-code lottery" is used in the UK to define the unfair differences in care received by patients who live in different regions. This term can be applied to Europe to define the difference in care in the 28 countries.
} 
have different weights and importance depending on the country (European Parliament, 2015). Refer to chapter 5 and 8 for more discussion on this.

International regulatory bodies in charge of the MAA process have developed new tools to accelerate patient access to promising new drugs (see chapters 4 and 5). Figure 1-4 presents the most important ones from the EU (by EMA) and USA (by FDA). In these new evaluation methods, the agencies evaluate data that was usually not accepted as sufficient evidence for an MAA. These methods use the available best evidence to weight the benefit/risk ratio and determine whether the drug is of high importance to patients and should be available faster through a 'Fast Track' 6 process rather than through the traditional procedure. Sadly, these efforts are in vane - in the EU- if national HTAs and national payers do not accept the same type of evidence for the reimbursement process. Some steps are being taken to improve the gap between the MAA and reimbursement process, such as the EMA-HTA joint Scientific Advice (European Medicine Agency, 2014); but many more efforts are needed in order to ensure early patient access to innovative and effective new medicines. This issue will be discussed in more detail in chapter 5 .

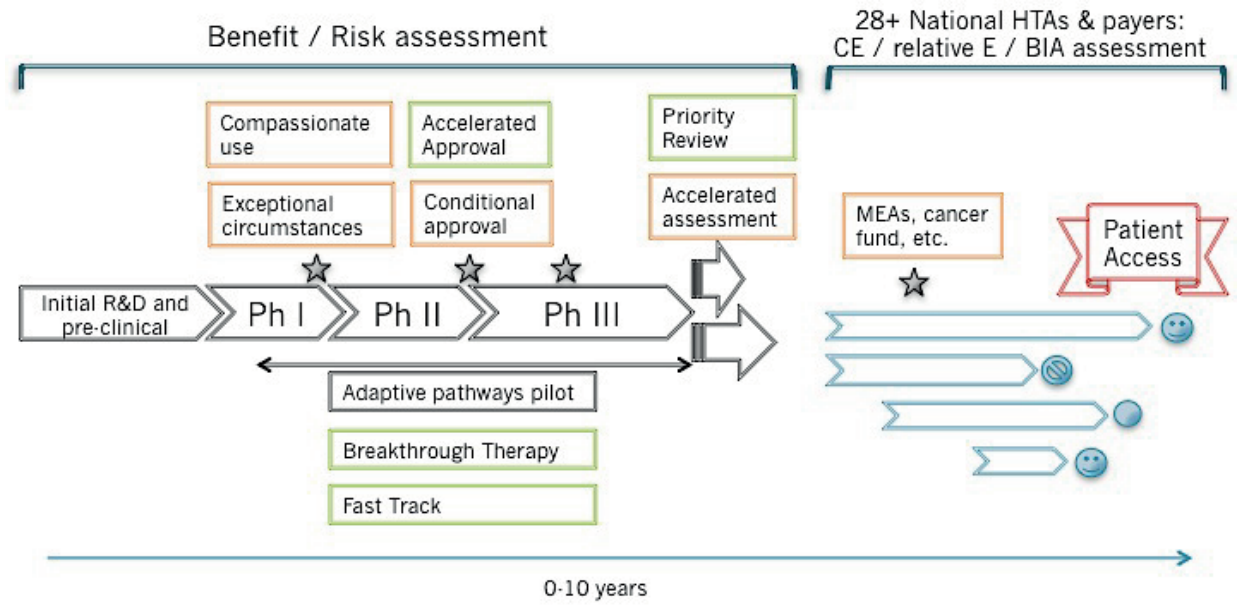

Figure 1-4. Available regulatory tools for early dialogue and early patient access placed on the current drug development phases from initial R\&D to the market. Orange boxes are for available programs in the EU and green boxes are for available programs in the USA (image from Chapter 4). BIA: budget impact analysis, CE: cost-effectiveness, HTAs: health technology assessment bodies, MEAs: managed entry agreements, $\mathrm{Ph}$ : phases in clinical trials., R\&D: research and development, relative E: relative effectiveness.

\footnotetext{
6 'Fast Track' here does not specifically refer to the FDA procedure with the same name, but rather as a general term to designate all procedures which are faster than the traditional MAA process.
} 


\subsection{Decision Supporting Tools}

Now that the concepts of personalised medicine and a drug's pathway from the lab to the patient have been introduced, we will discuss some of the available decision supporting tools for stakeholders involved in the development chain. Decision supporting tools in the context of this thesis refer to instruments available to policy makers and other stakeholders involved in the drug development process to take informed decisions. These tools produce, gather, analyse and present all the relevant information available and necessary to make decisions.

From the array of available decision supporting tools, the following will be discussed:

- Clinical trials: the information on drug safety, efficacy and effectiveness is gathered through clinical trials that test the drug on a certain patient population or healthy volunteers.

- Evaluation tools during the marketing authorization procedure: here all the methods and instruments used during the MAA are considered.

- Big data: newly developed technologies offer the potential to use a large array of heterogeneous data sets for decision making purposes.

- Game theory: the "mathematical models of conflict and cooperation between intelligent rational decision-makers" (Myerson, 1991) can be used in the drug development process to maximise the utility of the stakeholders throughout the whole process and study the interactions between all stakeholders involved, ultimately bringing an added value to patients and citizens.

\section{Clinical trials}

As already mentioned, clinical trials are essential in the drug development process to elucidate important safety, efficacy and effectiveness aspects from a medicine in a specific patient population. Firstly the drug's safety is investigated in healthy volunteers or terminal patients. Once an acceptable safety profile is established, efficacy and effectiveness are tested in the relevant patient population.

Most clinical trials are sponsored by pharmaceutical, biotechnological companies or other sponsors ${ }^{7}$ and carried out for MAA purposes. However, investigators ${ }^{8}$, not-for-profit organisations and national bodies also plan and carry out studies to

\footnotetext{
${ }^{7}$ Clinical Trial sponsors are defined in ICH-GCP as „an individual, company, institution, or organization which takes responsibility for the initiation, management, and/or financing of a clinical trial" (ICH-GCP 1996).

8 Investigators here refer to sponsor-investigators, defined by ICH-GCP as „an individual who both initiates and conducts, alone or with others, a clinical trial, and under whose immediate direction the investigational product is administered to, dispensed to, or used by a subject" (ICH-GCP 1996) and usually refers to a physician (although in some countries other professionals are also allowed to be sponsor).
} 
evaluate the safety and efficacy of a new intervention, a mix of existing treatments or an approved medicine in new populations. Traditionally drugs go through all phases of clinical trials ( $\mathrm{Ph}$ I, Ph II, Ph III ${ }^{9}$ ) before being submitted for an MAA; however, with the new evaluation methods and clinical trials designs, some of the phases are being merged (Ph I/II) and some MAAs are submitted with data limited to Phase I or phase II trials.

New clinical trial designs that move away from the traditional randomised controlled clinical trial (RCT) are emerging. The rise in the development of treatments for rare diseases (diseases with prevalence of less than 5 in 10,000 patients in the EU (EMA, 2007)) and personalised medicines has contributed to the new trial designs due to the small patient populations available.

Some examples of new trial designs are the so-called 'umbrella' or 'basket' trials and enrichment or adaptive design strategies. Clinical trial enrichment refers to trials that use biomarkers to select and include only the biomarker-positive or biomarker-negative population (FDA, 2010). This can be acceptable from an MAA perspective only if there is sufficient biological evidence on the relevance of the biomarker. If the enrichment strategy is adaptive, it is usually carried out at previously fixed interim analysis points. Umbrella trials encompass numerous subtrials where various drugs are tested on different mutations in one single disease within one single master protocol; the control group can be shared by all sub-trials. 'Lung-Map' is an example of umbrella clinical trial in squamous cell lung cancer in the USA (Lung-Map, 2017); whereas the 'National Lung Matrix Trial' runs in the UK in non-small cell lung cancer (Middleton, et al., 2015). On the other hand, basket trials test the efficacy and safety of one single drug on one single mutation in various cancer types; such as the on-going KEYNOTE-028 - NCT02054806 - clinical trial investigating the use of the anti-PD-1 agent pembrolizumab in multiple difficult to treat cancers (clinicatrials.gov, 2016). Even tough the differences are clear; the concepts of umbrella and basket trials are often interchanged in the scientific literature.

The FDA published in 2010 their "Guidance for Industry on Adaptive Design Clinical Trials for Drugs and Biologics" (FDA, 2010) to give guidance to involved stakeholders on the use of new trial designs for MAAs. Unfortunately, the EMA guidelines and reflections papers on this topic are out-dated. Their update should include new trial types and all the requisites that should be considered when designing clinical trials that include biomarker-based inclusion/exclusion criteria for an MAA.

Ultimately truly personalised medicines will need truly "personal" clinical trials. Some experts have proposed the N-of- 1 trials (Lillie, Patay, Diamant, Issell, Topoll, \& Schork, 2011), where each person acts as their own control. Some clinical trials

\footnotetext{
${ }^{9} \mathrm{Ph}$ I: Phase I
} 
for rare diseases are already tested this way (Stunnenberg, et al., 2015), whereby a baseline measurement is registered for each patient entering the trial with his/her usual treatment (or no treatment, or placebo), and then compared to measurements done with the test drug to conclude on risk/benefit (if testing new drug vs. placebo) or measure relative effectiveness (if using two marketed drugs or a new vs. a marketed drug).

\section{MAA Evaluation tools}

In the last decade, the EMA and FDA have introduced new approval pathways for promising medicines in order to accelerate their availability to patients. Conditional approval, accelerated assessment and the adaptive pathways pilot are some examples of EMA tools; while accelerate approval, priority review, breakthrough therapy and fast track are some of the pathways available at the FDA (see Figure 1-4). This will not be discussed in more detail here since the general MAA procedure at the EMA has been described above and these new methods are extensively described in chapters 4 and 5 .

\section{Big data}

An increase in quantity and variety of data alone would not be useful to the healthcare industry without the parallel improvement of storing capacities and more importantly without the development of stronger analytical tools. The use of big data in a variety of industries has seen a boom in recent years (e.g. in aviation, banking, insurances, etc.). Big data and data analytics are also being used more and more in the healthcare value chain including drug development, epidemiology, informed real-time patient treatment and public health policy-making. However, the full range of possibilities to all stakeholders (manufacturers, regulators, payers, healthcare providers, decision makers, researchers) has not been exploited yet. The transparent, responsible and ethical use of data for public health has the potential to improve general health outcomes. The potential use of big data, the available data sources and the main practical and ethical challenges are widely discussed in Chapter 6.

\section{Decision supporting tools and models from other disciplines}

Other scientific disciplines apply interesting decision supporting tools and decision models for their work and budget allocations. These methods could also help the various stakeholders in the healthcare sector, especially if they are modified according to the special needs of this sector. This work focuses on one of these tools that will be discussed in more detail in chapter 7 and is shortly introduced below: 


\section{Game theory}

Game theory models the decision making process in situations where outcomes are determined by one or more players' choices and/or chance. It is widely applied in a variety of fields such as philosophy, resource allocation and networking, biology, artificial intelligence, politics and of course economics (Leyton-Brown \& Shoham, 2008). Even though it helps to understand conflict and cooperation in decision making, it has not been widely applied to the drug development process so far. ${ }^{10}$ In chapter 7 a framework to apply game theory to the drug development process is proposed.

\subsection{Aim and outline of the thesis}

The thesis aims to:

a. Analyse the current landscape of personalised medicine in Europe,

b. evaluate the availability of regulatory tools to facilitate timely patient access, and

c. describe several decision supporting tools to facilitate a pan-European implementation of personalised medicine.

This thesis starts with the analysis of the current landscape, including three different dimensions: drug development, regulatory process and patient access. It analyses the strengths and opportunities of personalised medicine and the gaps and needs for its full implementation in Europe and beyond, including the description of the FP7 ${ }^{11}$ funded EC project PerMed which focused on Personalised Medicine and produced a Strategic Research and Innovation Agenda (SRIA). All this covers the first aim of describing the current landscape of PM.

For the second aim, the available tools to facilitate early patient access to promising medicines in the EU and USA are evaluated, from an MAA point of view. On this basis this work goes on to describe the need for HTAs to catch up with the EMA in Europe and create decision supporting tools that allow them to analyse less mature patient data than the traditional process requires. Finally, big data is discussed as a promising decision supporting tool for the drug development process as well as other public health uses, and especially for personalised medicines. And one decision supporting tool from other scientific disciplines (game theory) is analysed in detail and introduced for its use in decision making in the drug development process. As we have seen throughout the introduction, this thesis focuses on Europe.

\footnotetext{
10 This is to the best of our knowledge, since there are not many publications on the topic, but it might have been used by companies without publishing the models.

11 FP7 was the equivalent to the current European Union's Horizon2020 research and innovation funding program in 2007-2013.
} 
Even though some aspects from the USA and other regions are discussed, the reader should assume it addresses Europe if the region is not specified.

\section{Research questions}

The following research questions are central to this thesis:

I. What are the characteristics of the current landscape for Personalised Medicine?

a. What has already been proposed in the EU for Personalised Medicine (Chapter 3)?

b. What are the main strengths and opportunities (Chapter 2 and 3)?

c. What are the main gaps and needs for its implementation (Chapter 2 and 3)?

II. What decision supporting tools during the drug development process are available for PM and to facilitate timely patient access?

a. During the MAA procedure (Chapter 4)?

b. During the reimbursement process (Chapter 5)?

III. How can Big Data contribute as a decision supporting tool to public health and to the implementation of PM (Chapter 6)?

IV. How can other decision supporting tools (such as game theory) contribute to the public health value chain and drug development (Chapter 7)?

\section{Research design}

Mixed methods were used to answer the research questions. Each chapter describes the individual methods used for the material presented; therefore only a very short description of the methods will be described in this section.

I. To describe the current landscape of personalised medicine in Europe a systematic literature review was carried out. The texts identified through the literature review were analysed using the SWOT framework ${ }^{12}$ on personalised medicine as the main component of the analysis. After the SWOT analysis was completed a gaps and needs analysis was performed. This systematic literature review was complemented by semi-structures interviews to relevant stakeholders from different disciplines. Thanks to the heterogeneous mix of stakeholders who authored the analysed texts, and who were contacted during the interviews, the results offer the entire picture of personalised medicine across the healthcare spectrum and from all points of view.

${ }^{12}$ SWOT: Strengths, Weaknesses, opportunities and threats 
II. A secondment at the EMA facilitated the analysis of existing decision supporting tools in the EU and USA for the drug development process.

a. For the MAA procedure, publicly available documents and databases published by the EMA and FDA were analysed to identify the medicines that were approved using "conditional" approval methods that facilitated earlier patient access to medicines. Thereafter, publicly available documents, published after each marketing authorization (EPARs), were investigated to find the type of evidence used to approve the medicines.

b. The attendance to joint EMA-HTA meetings at the EMA helped to compare the methods used by the EMA during the MAA and the methods used by HTABs and payers during the reimbursement process and the difference in their requirements. This was complemented with a systematic search and analysis of official HTABs websites, guidelines, documents and relevant literature.

III. A literature search and discussions with relevant stakeholders were used to analyse the potential use of big data for public health purposes.

IV. Basic game theory concepts as well as the available literature on the application of game theory in other areas was analysed to propose a framework that facilitates the utilization of new decision supporting tools for public health decision making purposes, specially in the drug development process.

\section{Thesis outline}

This thesis has eight chapters. In the pages above the most important concepts for the thesis are introduced, such as the general concept of personalised medicine, the drug development process including patient access to drugs and the notion of decision supporting tools. Chapter 2 expands on personalised medicine, the current situation and the main obstacles for its implementation. The European FP7 funded project PerMed is further described in chapter 3 as well as the gaps ands needs analysis and SWOT performed under the project to index the barriers to personalised medicine. The use of tools to facilitate early patient access to promising medicines is described in chapter 4 with an analysis that compares the situation in the EU and USA. Chapter 5 extends on the concept of early patient access and discusses the challenge faced by HTAs due to the new MAA tools implemented by the EMA and the urgent need to catch up so that the ultimate goal of early patient access is accomplished. Finally, decision supporting tools are described in chapter 6 and 7, with the description of the use of big data and game theory in the healthcare value chain respectively. Chapter 8 synthesizes all topics into a general discussion, touching on the challenges and limitations and finally drawing conclusions on the implementation of personalised medicine with the aid of various decision supporting tools in Europe and beyond. 


\section{References}

Brand, A. (2009). Integrative genomics, personal genome tests and personalised healthcare: the future is being built today. European Journal of Human Genetics, 17 (8), 977-978.

Castiglione, F., Tier, P., Ernst, M., \& et al. (2013). The Onset of Type 2 Diabetes: Proposal for a MultiScale Model. JMIR Research Protocols, 2 (2), S. e44.

clinicatrials.gov. (2. February 2016). NCT02054806: Study of Pembrolizumab (MK-3475) in Participants With Advanced Solid Tumors (MK-3475-028/KEYNOTE-28). Viewed on 16. February 2016 at https://clinicaltrials.gov/ct2/show/NCT02054806

Council of the European Union. (2015). Council conclusions on Personalised medicine for patients -7 December 2015. Brussels: General Secretariat of the Council.

CSA PerMed: PerMed: Shaping Europe's Vision for Personalised Medicine. Strategic Research and Innovation Agenda (SRIA). Cologne, German Aerospace Center (DLR), 2015. http://www.permed2020.eu/_media/PerMed_SRIA.pdf.

EMA. (3. July 2007). Orphan drugs and rare diseases at a glance. Viewed on 16. February 2015 at www.ema.europa.eu: http://www.ema.europa.eu/docs/en_GB/document_library/Other/2010/01/WC500069805.pdf

European Commission. (2013). Use of '-omics' technologies in the development of personalised medicine. Brussels: European Commission.

European Commission. (2001). Abgerufen am 02. February 2016 von Clinical Trial Directive (Directive 2001/20/EC): http://ec.europa.eu/health/human-use/clinical-trials/directive/index_en.htm

European Commission. (2014). Abgerufen am 02. February 2016 von new European Clinical Trial Regulation (Regulation EU No 536/2014): http://ec.europa.eu/health/human-use/clinicaltrials/regulation/index_en.htm

European Medicine Agency. (7. May 2014). www.ema.europa.eu. Viewed on 15. February 2016 at Best Practice guidance for Pilot EMA HTA Parallel Scientific 5 Advice procedures: http://www.ema.europa.eu/docs/en_GB/document_library/Regulatory_and_procedural_guideline /2014/05/WC500166226.pdf

European Parliament. (2015). Towards a Harmonised EU Assessment of the Added Therapeutic Value of Medicines. Policy Department A: Economic and Scientific Policy. Brussels: European Union.

Ferrario, A., \& Kanavos, P. (2013). Managed entry agreements for pharmaceuticals: the European experience. Brussels: EMiNet.

FDA. (2010). Adaptive Design Clinical Trials for Drugs and Biologics. Washington D.C.: U.S. Department of Health and Human Services.

Francioso, S., Almerighi, C., Forte, P., Bandiera, F., Angelico, M., \& et al. (2014). A simple rule to personalize standard dual therapy across all genotypes in naive chronic hepatitis $C$ patients: The TT4 randomized trial. Digestive and Liver Disease, 46 (2), S. 164-169.

Gartlehner, G., Hansen, R., Nissman, D., \& al., e. (2006). Criteria for Distinguishing Effectiveness From Efficacy Trials in Systematic Reviews. Rockville (MD): Technical Reviews No 12.

ICH. (2016). ICH Safety Guidelines S1-S11. International Conference on Harmonization.

Kanavos, P., \& Angelis, A. (2013). Multiple Criteria Decision Analysis for Value Based Assessment of New Medical Technologies: A Conceptual Framework. London: LSE Health.

Lung-Map. (2017). www.lung-map.org. Abgerufen am 25. Apr 2017 von About lung-map: https://www.lung-map.org

Lehrach, H. (2015). Virtual Clinical Trials, an essential step in increasing the effectiveness of the drug development process. Public Health Genomics, 18, 366-371.

Leyton-Brown, K., \& Shoham, Y. (2008). Essentials of Game Theory. Morgan and Claypool Publishers.

Lillie, E. O., Patay, B., Diamant, J., Issell, B., Topoll, E. J., \& Schork, N. J. (2011). The n-of-1 clinical trial: the ultimate strategy for individualizing medicine? Per Med, 8 (2), 161-173.

Martinalbo, J., Bowen, D., Camarero, J., Chapelin, M., Démolis, P., Foggi, P., et al. (2016). Early market access of cancer drugs in the EU. Annals of Oncology, 27 (1), 96-105. 


\section{Chapter 1}

Middleton, G., Crack, L., Popat, S., Swanton, C., Hollingsworth, S., Buller, R., et al. (2015). The National Lung Matrix Trial: translating the biology of stratification in advanced non-small-cell lung cancer. Annals of Oncology, 26 (12), 2464-9.

Munoz, J., \& Kurzrock, R. (2012). Targeted therapy in rare cancers - adopting the orphans. Nature Reviews Clinical Oncology, 9, 631-642.

Myerson, R. B. (1991). Game Theory: Analysis of Conflict. Harvard University Press.

NICE. (19. Feb 2015). www.nice.org.uk. Viewed on 25. Apr 2017 at https://www.nice.org.uk/ news/blog/carrying-nice-over-the-threshold

Nordon, C., Karcher, H., Groenwold, R., Zöllned Ankarfeldt, M., Pichler, F., Chevrou-Severac, H. Abenhaim, L. (2016, January). The "Efficacy-Effectiveness Gap": Historical Background and Current Conceptualization. Value in Health, 19(1), 75-81.

Owens, S. R., \& Breithaupt, H. (2002). From genomes to cures - a long way to go. EMBO reports, 3 (1), 11-14.

Personalized Medicine Coalition. (2011). The Case for Personalized Medicine, 3rd edition. New York City: Personalized Medicine Coalition.

Personalized Medicine Coalition. (2016). 2016 Progress Report: Personalized Medicine at FDA. Personalized Medicine Coalition.

Pingle, S., Sultana, Z., Pastorino, S., Jiang, P., Mukthavaram, R., Chao, Y., et al. (2014). In silico modeling predicts drug sensitivity of patient-derived cancer cells. Journal of Translational Medicine, 12 (128).

Schneider, C. K., \& Schäffner-Dallmann, G. (November 2008). Typical pitfalls in applications for marketing authorization of biotechnological products in Europe. Nature Reviews Drug Discovery, 893-899.

Stunnenberg, B., Woertman, W., Raaphorst, J., Statland, J., Griggs, R., Timmermans, J., et al. (2015). Combined $\mathrm{N}$-of-1 trials to investigate mexiletine in non-dystrophic myotonia using a Bayesian approach; study rationale and protocol. BMC Neurology, 15 (43).

Touraine, M. (2016). Press conference following the serious accident in the framework of a clinical trial. http://social-sante.gouv.fr. Rennes: 15 January 2016.

Trials, E. G. (2006). Expert Group on Phase One Clinical Trials: Final report. UK: TSO (The Stationery Office). 


\section{Chapter 2}

\section{Working towards personalization in medicine: main obstacles to reaching this vision from today's perspective}

Published as: Leyens L, Horgan D, Lal J A, Steinhausen K, Satyamoorthy K, Brand A (2014). Working towards personalization in medicine: main obstacles to reaching this vision from today's perspective. Personalized Medicine. Vol. 11, No. 7, Pages 641-649, (doi:10.2217/pme.14.55) 


\title{
Chapter 2
}

\begin{abstract}
Rapid advances in 'omics' sciences and technologies have elevated the relevance of personalized medicine. This article reviews the current advances in the application of personalized medicine, outlines and summarizes the key areas that still need to be addressed and gives recommendations in this direction. Eighteen relevant highlevel reports on personalized medicine were reviewed in order to identify the gaps and needs that are present for the implementation of personalized medicine. We identify 12 key areas that represent the main obstacles on the road towards the personalization of medicine and divide these 12 key areas into four domains, namely: scientific research and stakeholder collaboration; translational tools; regulations and systematic early dialog with regulators; and uptake into healthcare systems. All of the evaluated reports agree on the imperative need for intensive collaboration among all stakeholders with early active participation and changes in the current healthcare infrastructure.
\end{abstract}




\section{Introduction}

Genomics, transcriptomics, proteomics and metabolomics are all 'omics' sciences that are thriving, pushing the personalization of medicine forward. Even though the concept of personalized medicine has been around for over 2000 years Hippocrates considered it "...far more important to know what person the disease has than what disease the person has" - the developments in the omics sciences in the past decades have enabled a rapid progression towards the concept of universally accessible, truly personalized medicine.

The personalization of medicine is a mission. However, the journey has only just started, and although we have made good progress since the approval of Herceptin ${ }^{\circledR}$ (Roche Pharma AG, Grenzach-Wyhlen, Germany) for the treatment of HER2 ${ }^{+}$metastatic breast cancer 16 years ago, there are still many obstacles to conquer in order to reach the target of truly personalized medicine. Over 100 approved drugs in the USA contain information on biomarkers in their label (Figure 1) and over 30 targeted drugs have been approved based on the use of different biomarkers in order to assign the appropriate treatment to each patient individually by selecting the correct population or adjusting the dose, resulting in improved efficacy and improved safety profiles [1].

Personalized treatment technologies are already being applied in several fields with success. Cancer and rare diseases are the best-known examples, but other fields, such as nutrition [2,3], psychology, cardiology [4] and infectious diseases $[5,6]$, have also successfully applied the concept of personalized medicine for prevention, diagnosis, correct dosing, prediction of differential response and adverse event prevention. Besides, other interesting applications are being pursued, such as the in silico models that predict drug sensitivity of patient-derived cancer cells a priori, which could be used to stratify patients in clinical trials and for treatment decision making in clinical practice [7]. 

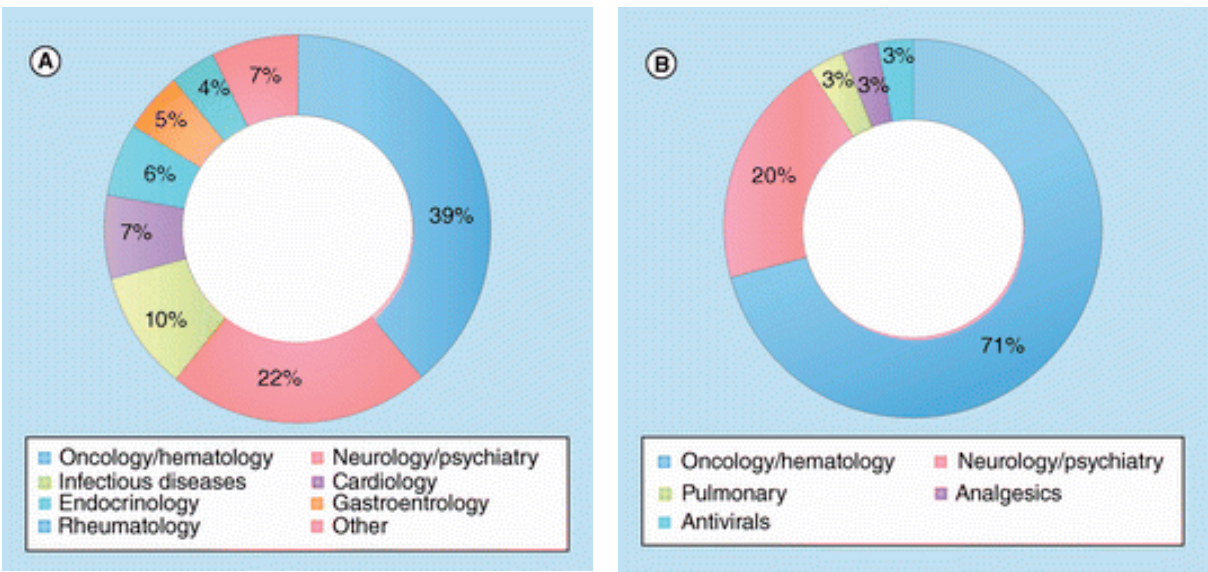

Figure 1. Pharmacogemics information and guidance in approved medicines.

(A) Pharmacogenomics biomarker information in drug labeling per therapeutic area. (B) Drugs approved with specific actionable guidance in labeling, classified per therapeutic area $(n=34)$. Data taken from [1].

In this article, we will discuss the main obstacles that lay ahead. Many reports have already been published on the topic of personalized medicine, analyzing the current landscape and pointing out the gaps and needs. We have reviewed 18 relevant reports and identified the gaps and needs that they describe as essential in the road towards the personalization of healthcare; part of this review was conducted within the CSA PerMed project [8]. The reviewed reports have been written by a variety of authors, ranging from the European Commission, regulatory agencies and national parliaments through to companies, alliances on personalized medicine, patient associations and a variety of European working groups. Independent of background, authors, main focus, main interests and the audiences they are directed to, most of the reports shared similar views on what still needs to be done. This article will present and discuss the most relevant and most discussed topics in the areas of scientific research and stakeholder collaboration, translational tools, early dialog with regulators, legislations and uptake into healthcare systems.

The following section will lay out the gaps and needs identified by the reports; each report will not be referenced directly in the text, so please refer to Table 1 for a list of consulted reports. The framework presented in Figure 2 depicts the gaps and needs that have been repeated by a large proportion of the reports, always keeping in mind that the emphasis is on scientific research and collaboration, translational tools, early dialog, legislations and uptake into healthcare systems. The discussion will be focused on what we consider the 12 'essential' needs, including proposals for action and already existing examples of good practice. 


\section{Changing current systems and gold standards}

\section{Scientific research \& stakeholder collaboration}

\section{Targeted research}

We need to go a long way with regards to our knowledge of the mechanisms and causes of disease, the relationship between nature and nurture and all of the factors implicated in a condition (including the epigenome and external factors, among others). Advances in the omics sciences (transcriptomics, proteomics and metabolomics, and so on) and in systems biology could provide the final complete picture of disease characterization. Once the biological bases are known, treatment options can be explored and biomarkers can be identified and characterized. Industry, academia and society need to agree on priorities reflecting the greatest unmet needs and cutting across individual therapeutic priorities for treatment development.

\section{Multidisciplinary collaboration}

Most reports agree upon the fact that there is not enough collaboration across sciences, networks and stages of the development chain. There is a clear need for cross-disciplinary and cross-border collaboration in research and in drug development. Especially in personalized medicine, the collaboration of specialists from a variety of disciplines (basic researchers, physicians, geneticists, bioinformatics, counselors and ethicists, among others) is essential in order to reach the final treatment decision for the patient.

An essential element in order to facilitate collaboration across disciplines is promoting data sharing. Today, data often sits in disconnected silos guarded with secrecy by companies and research groups. To move away from this scenario, appropriate infrastructures for the collection, storage, management, sharing, mining, processing and analyzing of health data with appropriate quality controls and maintenance are needed. Even though a minimum of compatibility and comparability between datasets is desired, it is no longer compulsory: the latest data-mining techniques make the analysis of unstructured data possible [27].

We also have to identify what is relevant information and integrate it effectively in order to produce interpretations of an individual's health status through the development of new decision support tools (Information and Communication Technologies) and methodologies in order to analyze and interpret patients' data. To facilitate this task, we should ensure the universal adoption of electronic health records (including omics data and other determinants) and explore the integration 
of user-generated data into the health information collected by medical professionals.

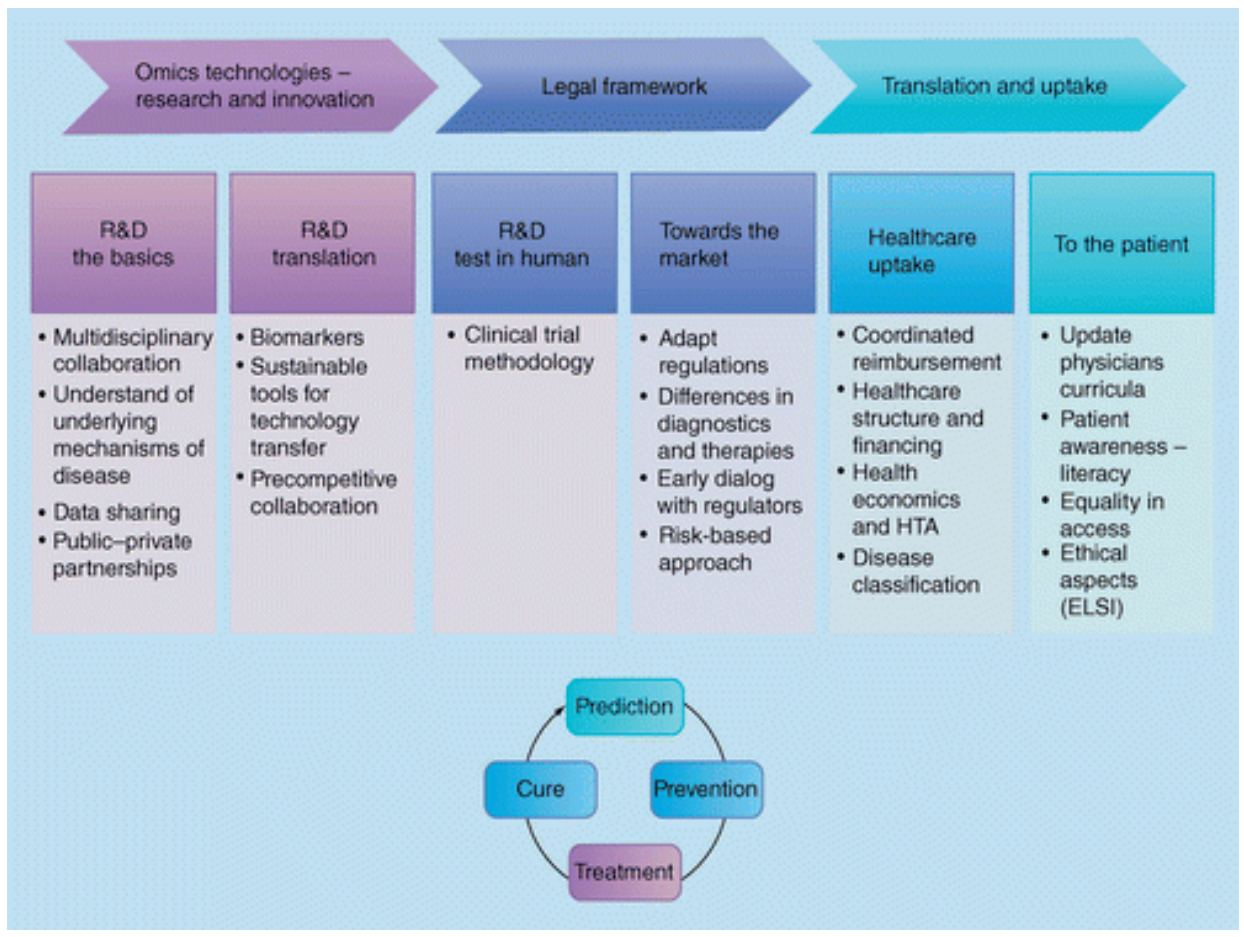

Figure 2. Gaps and needs for the personalization of medicine.

ELSI: Ethical, Legal and Social Implications HTA: Health Technology Assessment; R\&D: Research and development. Adapted from [15].

\section{Translational tools}

\section{Precompetitive collaboration}

Personalized treatments often rely on the combination of technologies, such as companion diagnostics (CDx) and drugs. For their successful development, we need to move from the current unidirectional approach of healthcare companies to a bidirectional approach involving an increased collaborative culture throughout the sector based around shared resources, the dynamic flow of ideas and interchange and knowledge transfer between companies. Pharmaceutical and medical device companies, as well as other actors in therapeutics development, should share competencies, expertise and resources. 


\section{Technology transfer}

The current tools for technology transfer into the market, and eventually into the healthcare system, are slow and inefficient and are becoming obsolete for medicinal products and therapeutics. For personalized medicine, new dynamic and sustainable pathways are needed in order to secure the timely and cost-effective translation of innovative technologies and knowledge into health polices and healthcare. Such pathways need to consider the new industrial, societal, legal and Intellectual Property constraints and ensure that safe and efficient treatments of good quality are put into the market. The correct functioning of high-quality CDx for the provision of understandable information to physicians is also inherent to personalized medicine. Some proposed models for real-time integration into healthcare, such as the learning-adapting-leveling model, combine technology transfer tools and public health assessment tools in order to increase the probability of success in drug development, reduce the overall costs of new medicines and provide quicker patient access [28].

\section{Clinical trials}

Clinical trials with thousands of patients, such as current pivotal studies for marketing authorization applications, will no longer be possible due to the small patient pools inherent to individualized medicine. New clinical trial methodologies with adaptive designs need to be applied in order to test personalized treatments. More and more examples of these trials are being carried out, especially in cancer trials with several drugs that are applied depending on the specific mutation expressed by each patient's tumor [29]. Since personalized medicine relies on the knowledge of the underlying mechanisms of disease, treatments targeted to the involved biological pathways will have lower safety issues and risks for patients than the ones associated to 'one-size-fits-all' blockbuster medicines.

' $n=1$ ' trials have also been proposed, with each citizen acting as its own control over his/her lifetime. They are based on the fact that genetic and health information from individuals is dynamic and changes throughout the life course of a person. New models of disease with analytical methods and modeling approaches that take this dynamism of information into account are necessary in order to combine all of the knowledge from research and bring it to physicians so as to assist in the decision making process. Models such as 'computerized twins of patients' or 'metabolic identity cards' could be very helpful decision-support tools.

\section{Regulations \& systematic early dialog with regulators}

\section{Systematic early dialog}

Regulators should provide guidance, clarity and predictability to industry. Early dialog between manufacturers and decision-makers throughout all of the 
regulatory steps can resolve the misalignment of expectations between innovators, regulators and payers, leading to a more efficient drug development process. This will also help identify the requirements from regulators, avoid the duplication of efforts and research and identify the best reimbursement mechanisms early in the development process. The 'scientific advice' meeting and the 'breakthrough therapy program' at the EMA and US FDA, respectively, offer a platform for this early dialog. Another example for Europe is the Innovative Medicine Initiative (IMI), the biggest public-private partnership in biopharmaceutical research and innovation.

\section{Update \& adapt regulations}

Current regulations are outdated and inappropriate if we are aiming to move towards the personalization of medicine. In Europe, several regulations are under revision (e.g., for data protection and medical devices) or have been recently approved by the parliament (e.g., the clinical trial regulation), with changes that will impact the future of personalized medicine. Simplified, harmonized (EU), coherent (across directives) and predictable regulatory procedures that take into account the particularities of personalized medicine are necessary.

Some examples of needed changes are as follows:

- The introduction of parallel approval of diagnostics and drugs;

- The introduction of risk-based approaches for market approval;

- Prioritizing individual benefit and personal utility in decision making;

- Real-time data and observational studies need universal acceptance and application;

- Stronger postmarket surveillance strategies are needed in order to identify rare adverse events;

- A change in the marketing approval process for drugs is needed in the long term. For example, take the decrease in the number of patients in clinical trials on personalized treatments compared with current pivotal clinical trials, giving less safety and efficacy information at the time of marketing authorization applications.

- A risk-based regulatory process with de-risking processes in place is necessary in order to share the risks between companies and regulatory agencies (as introduced in the newly proposed adaptive licensing by EMA and the European Commission [30,31]). 


\section{Uptake into healthcare systems}

\section{Coordinated reimbursement process}

A survey in $27 \mathrm{EU}$ countries regarding the reimbursement of trastuzumab (for the treatment of $\mathrm{HER}^{+}$breast cancer) and its diagnostic testing revealed highly fragmented provider and payer structures in different Member States (MSs), with clear problems at the interface between different funding streams and the combination of drugs and diagnosis in a treatment [32]. As we have seen in this example, the uncoordinated reimbursement process for drug-diagnostic combinations, as well as the fragmented Health Technology Assessment (HTA), pricing and reimbursement processes across MSs, make the market entry and uptake of personalized technologies very difficult. We should move towards a smooth and coordinated reimbursement process that considers the complexities of drug and medical device combinations, including an increased coordination between institutions involved in pricing, paying and providing treatments.

\section{Healthcare systems \& models}

Personalized medicine can only flourish by fitting into the healthcare systems that are currently in place; however, there are certain aspects that need to change or progress for citizens to enjoy its full potential (e.g., attitudes, structures and models of providers, cost assumptions, patient behaviors and interests and a focus towards preventative care). A general change in mindset in healthcare delivery and provision is needed in order to catch up with the advances in science. Most importantly, budgets and processes in healthcare systems are not designed for drug-diagnostic combinations, with the current fragmented financing and delivery system offering very limited rewards for integrated solutions and not integrating the 'full cost of the patient' perspective. A long-term investment perspective rather than the current focus on short-term savings is certainly needed. Moreover, we need an overall healthcare financing strategy, with innovative mechanisms such as risk and benefit sharing, profit reinvestment, value-based pricing and managed entry agreements, as well as the introduction of incentives for the adoption of personalized medicine and to recognize and reward innovation.

The personalization of medicine will require special infrastructures for diagnosis and treatment that are not available at most healthcare providers. Systems will need to adapt to chronic care requirements and preventative strategies and adopt an inter- and multi-disciplinary approach at the provider level. This may result in a complete reorganization of the healthcare system in the long term, with the creation of multidisciplinary teams and specialists in personal healthcare and medical specialties possibly moving away from an organ-based structure to a systems-based structure. 


\section{Chapter 2}

The collaboration and integration between the different levels of providers and different welfare systems, such as primary care and hospitals or health and social care services, together with an increased offer of personalized wellness and preventative approaches by healthcare providers, such as in nutrition, could have huge impacts on the future morbidity of certain conditions, leading to large cost savings for the whole system.

\section{Disease classification}

With the new information obtained from the omics sciences, it has become evident that the current symptom- and phenotype-based disease classification system needs to shift towards more genetically defined grouping or 'diseasomes', taking into account complex biological systems and pathways (Figure 3). This classification may have large economic and organizational impacts that should not be underestimated.

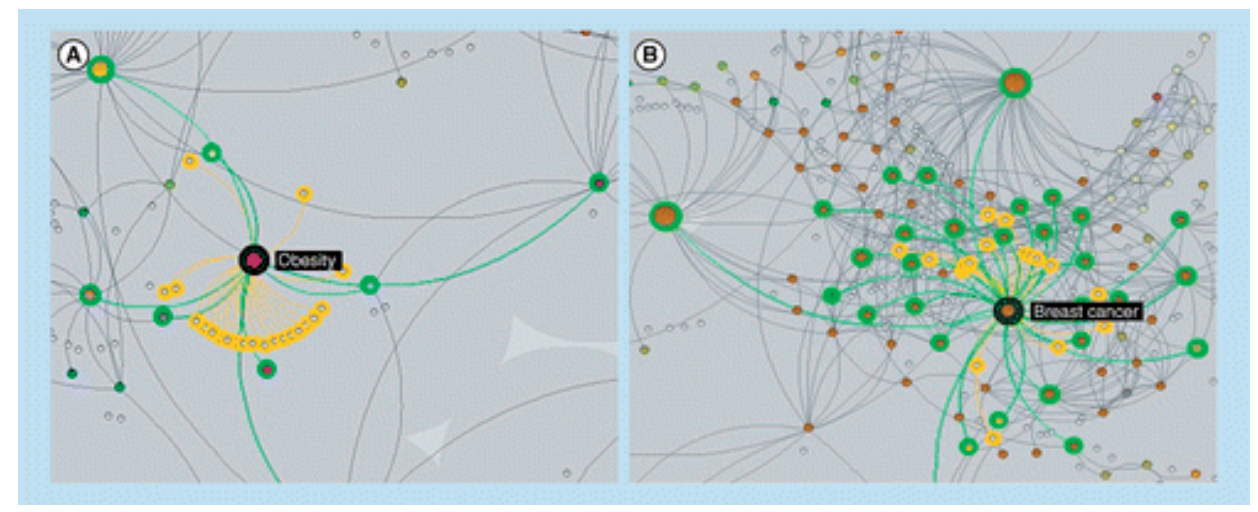

Figure 3. Disease classification system based on genetically defined grouping.

Diseasome map of (A) obesity and (B) breast cancer, with related genes (yellow lines and circles) and conditions (green lines and circles).

Reproduced with permission from [33].

\section{Physicians' curricula}

Low uptake of personalized treatments is nurtured on the one hand by physicians, who completely ignore the availability of modern diagnostics and personalized treatment options, and on the other hand by the overload of unstructured information in the clinical implementation of $\mathrm{CDx}$ and personalized treatments, nourished by unclear labeling and the lack of support tools for treatment decisions. Potential solutions could be to ensure accurate product labeling, the development of decision-support tools to guide diagnosis and treatment and the creation of universal, flexible and adaptable guidelines for the implementation of personalized medicine into healthcare. 
Healthcare professionals lack sufficient literacy and awareness of personalized treatment approaches, genomics and information technologies. The outdated curricula of their training, especially of physicians, should be revised in order to provide teachings regarding all of the aspects that are necessary for the implementation of personalized medicine, including (but not limited to) CDx-drug therapies, ICT, cross-disciplinary interactions, patient communication (due to the new patient-doctor relationship) and scientific and clinical applications of genomics and proteomics.

\section{Patient literacy \& awareness}

In the past decade, patients have become increasingly active managers of their own health. In person-centered healthcare, the role of proactive consumers of health ('prosumers') is essential and will also shape the relationship between medical professionals and patients, which is currently based on agency, towards a more collaborative partnership. Moreover, informed prosumers can play a larger role in the healthcare ecosystem and participate in decision making activities. Patients could be directly involved in medical research through the use of patient information and records in order to inform the next generation of successful therapies. Some examples of innovative platforms that involve patient data sharing and promote data ownership are PatientsLikeMe, Reg4All, Health Data Cooperative and CrowdMed, among others.

In order to make this vision possible, we should recognize the rights of patients to seek information, promote health literacy and develop education and communication strategies with the use of media technologies. Despite never having enjoyed such easy access to health information, not all of the accessible information is accurate. The correct use and spread of the latest scientific and technological information could be promoted by creating public reference websites, with up-to-date information for patients about available diagnostics, personalized treatments and their risk/benefit evaluation, providers and the use of genetic information.

\section{Ethical aspects \& conclusion}

The change in the paradigm of healthcare proposed by all of these reports has huge socioeconomic implications. Exhaustive social research on the consequences of the personalization of medicine for society and patients remains to be carried out, and we need to ensure that they translate directly to benefits for individual citizens and society. All of the reports reached a consensus in expressing the need to ensure equality in access to personalized therapeutics; they should not become a luxurious commodity of the rich. 
The ethical challenges brought about by personalized medicine, genomics and the advances in medical technologies are large; they confront society with new questions and new aspects that have never been dealt with before in different fields, such as in health data. One of the essential steps is to correct the perceptions of genetic exceptionalism: all health data and information have to be treated in the same way and as a whole, including genetic information. Besides the fact that the complexity and dynamism of genetic information makes the possibility of its malicious use rather remote, we can exploit modern ICT and create sensible regulations in order to protect personal health data. Furthermore, it is imperative to put mechanisms in place in order to protect patient privacy, patient choice and avoid a medical 'big brother' and overbearing medical surveillance.

As seen in the 12 subheadings discussed above, it is imperative to promote the increased collaboration between stakeholders and healthcare actors (including patients) across sciences, sectors and borders. The early engagement of researchers, patients, policy-makers, regulators and payers can lead to the timely translation of personalized treatment options into healthcare in a cost-efficient manner. Cost-benefit and cost-effectiveness analysis will be needed in all areas where personalized medicine is being applied in order to prove numerically that an approach of the personalization of medicine is cost effective in the long term.

All of these aspects can be summarized in the concept of $\mathrm{P} 4$ health: predictive, preventative, personalized and participatory health. All stakeholders need to focus on adding value and doing so affordably for healthcare systems, without forgetting the ultimate goal of personalized medicine: deliver new treatments at reasonable cost that have significant impacts in treating or preventing diseases with improved effectiveness, reduced toxicity, reduced cost and increased cost-effectiveness than the already-available interventions.

\section{Future perspective}

Several reports and literature articles point towards the key areas we have addressed. There has been progress towards these areas as new initiatives and programs arise from the EU, member states, industry, nongovernmental organizations and patient groups, among others. There is still a long way to go given the complexity of all of the stakeholders involved, the different existing national healthcare systems and current fragmented data silos. Nevertheless, we believe that, given the exponential media attention paid towards personalized medicine, the published literature, the EC commitment and national government initiatives in place (with new ones on the horizon) and the developed alliances in personalized medicine, it seems likely a large share of personalized medicine 
applications will be in use in the next 10 years, provided that the 12 key points we discuss in this article are addressed and lobbied.

Table 1. List of consulted reports on personalized medicine to identify the gaps and needs in the road towards personalization of medicine (listed in alphabetical order of authors).

\begin{tabular}{ll}
\hline Name of author (year of publication) & Ref. \\
\hline Brand A (2012) & {$[9]$} \\
Brand A. and Lal JA (2012) & {$[10]$} \\
EAPM (2013) & {$[11]$} \\
EAPM (2013) & {$[12]$} \\
EFPIA (2013) & {$[13]$} \\
European Commission (2012) & {$[14]$} \\
European Commission (2013) & {$[15]$} \\
European Science Foundation (2012) & {$[16]$} \\
eHealth Task Force (2012) & {$[17]$} \\
F. Hoffmann-La Roche Ltd. (2011) & {$[18]$} \\
US FDA (2013) & {$[1]$} \\
Federal Ministry of Education and Research (BMBF), Germany (2013) & {$[19]$} \\
HOPE and PwC (2011) & {$[20]$} \\
iNNOVAHEALTH. Cyprus EU Presidency (2012). & {$[21]$} \\
Instituto Roche (2013). & {$[22]$} \\
l'Office parlementaire d'évaluation des choix scientifiques et technologiques (2014) & {$[23]$} \\
OPTI y Genoma España (2009) & {$[24]$} \\
TAB (2008) & {$[25]$} \\
Technology Strategy Board. Stratified Medicine in the UK. Vision and Roadmap (2011) & {$[26]$} \\
\hline
\end{tabular}




\section{References}

Papers of special note have been highlighted as: $\bullet$ of interest

1. US FDA. Paving the Way for Personalised Medicine. FDA's Role in a New Era of Medical Product Development. US FDA, MD, USA (2013).

2. Castiglione F, Tieri PM, De Graaf A et al. The onset of Type 2 diabetes: proposal for a multi-scale model. JMIR Res. Protoc. 2(2), e44 (2013). [CrossRef]

3. van Schalkwijk DB, De Graaf AA, Tsivtsivadze $E$ et al. Lipoprotein metabolism indicators improve cardiovascular risk prediction. PLOS ONE 9(3), e92840 (2014). [CrossRef] [Medline]

4. Turner RM, Pirmohamed M. Cardiovascular pharmacogenomics: expectations and practical benefits. Clin. Pharmacol. Ther. 95(3), 281-293 (2014). [CrossRef] [Medline] [CAS]

5. Ge D, Fellay JA, Thompson AJ et al. Genetic variation in IL28B predicts hepatitis C treatmentinduced viral clearance. Nature 461(7262), 399-401 (2009). [CrossRef] [Medline] [CAS]

6. Francioso $S$, Almerighi $C P$, Forte $P$ et al. A simple rule to personalize standard dual therapy across all genotypes in naive chronic hepatitis C patients: the TT4 randomized trial. Dig. Liver Dis. 46(2), 164-169 (2014). [CrossRef] [Medline]

7. Pingle S, Sultana $Z$, Pastorino $S$ et al. In silico modeling predicts drug sensitivity of patient-derived cancer cells. J. Transl. Med. 12, 128 (2014). [CrossRef] [Medline]

8. Per Med. www.permed2020.eu.

9. Brand A. Public health genomics and personalized healthcare: a pipeline from cell to society. Drug Metab. Drug Interact. 27, 121-123 (2012). [Medline] [CAS]

10. - Focused and concise article on the changes that need to be implemented by member states to use genome-based information technologies, which are essential for personalized medicine.

Brand A, Lal JA. European best practice guidelines for quality assurance, provision and use of genome-based information technologies: the 2012 Decalaration of Rome. Drug Metabol. Drug Interact. 27(3), 177-182 (2012). [Medline] [CAS]

11. - Offers a very good and exhaustive overview and analysis of personalized medicine, its benefits and the challenges faced for its implementation.

EAPM. Innovation and Patient Access to Personalized Medicine. European Alliance for Personalised Medicine, Belgium (2013).

12. EAPM. Big Data Workshop. 16th European Health Forum Gastein. Gastein, Austria, 2-4 October 2013.

13. - Good analysis and proposition for a strategic research agenda for personalized medicine.

European Federation of Pharmacetical Industries and Associations. The Right Prevention and Treatment for the Right Patient at the Right Time. Outline Strategic Research Agenda for Abiomedical Research Public Private Partnership under Horizon 2020. European Federation of Pharmacetical Industries and Associations, Belgium (2013).

14. European Commission, Biobanks for Europe.A challenge for governance. 2012. http://bookshop. europa.eu/en/biobanks-for-europe-pbKINA25302/.

15. European Commission. Use of '-Omics' Technologies in the Development of Personalised Medicine. European Commission, Belgium (2013).

16. • Offers a very good overview of personalized medicine.

European Science Foundation, Forward Look, Personalized Medicine for the European Citizen. Towards more precise medicine for the diagnosis, treatment and prevention of disease (iPM). 2012. www.esf.org/uploads/media/Personalised_Medicine.pdf.

17. eHealth Task Force. Redesigning Health in Europe for 2020. European Union, Luxembourg (2012).

18. F. Hoffmann-La Roche Ltd, Personalised Healthcare. Small difference, big effects. October 2011. www.roche.com/phc_brochure.pdf.

19. Federal Ministry of Education and Research(BMBF). Personalised Medicine - Action Plan, a new approach in research and health care. Germany. (2013). 
20. European Hospital and Healthcare Federation (HOPE). Personalized Medicine in European Hospitals. PriceWaterhouseCoopers. London, UK (2011).www.efpia.eu/documents/92/61/BIG-DATAWorkshop-16th-European-Health-Forum-Gastein-Report.

21. iNNOVAHEALTH. Building on Open Innovation Ecosystem in Europe for Healthcare. Cyprus EU Presidency, Cyprus (2012).

22. Instituto Roche, La Medicina Individualizada como oportunidad para el Sistema Nacional de Salud. 2013. www.institutoroche.es.

23. l'Office parlementaire d'évaluation des choix scientifiques et technologiques. Raport provisoire: les progrès de la génétique, vers unemédecine de précision ? les enjeux scientifiques technologiques, sociaux et éthiques de la médecine personnalisée. 22 January 2014. www.assembleenationale.fr/14/pdf/rap-off/i1724.pdf.

24. OPTI y Genoma España, Farmacogenómica: Medicina Personalizada y Predictiva. Informe de prospectiva Tecnológica Sectorial. Genoma España, April 2009. www.opti.org/publicaciones/ pdf/texto123.pdf.

25. TAB, Individualisierte Medizin und Gesundheitssystem. Zukunftsreport. Büro für TechnikfolgenAbschätzung beim Deutschen Bundestag, Germany (2008).

26. Technology Strategy Board. Stratified Medicine in the UK. Vision and Roadmap, UK (2011).

27. - Offers insights into unstructured data mining, which will become essential in personalized medicine.

Roque F, Jensen PH, Schmock, et al. Using electronic patient records to discover disease correlations and stratify patient cohorts. PLoS Comput. Biol. 7 (8), e1002141 (2011). [CrossRef] [Medline] [CAS]

28. - Proposal of an innovative technology transfer model.

Lal J, Vaidya A, Gutierrez-Iberluzea I, Dauben HP, Brand A. The learning-adapting-leveling model: from theory to hypothesis of steps for implementation of basic genome-based evidence in personalized medicine. Pers. Med. 10(7), 683-701 (2013). [Abstract] [CAS]

29. Wason J, Marshall A, Dunn J, Stein RC, Stallard N. Adaptive designs for clinical trials assessing biomarker-guided treatment strategies. Br. J. Cancer 110(8), 1950-1957 (2014). [CrossRef] [Medline] [CAS]

30. - Description of the newly proposed adative licensing model in Europe, which could play an important role for personalized treatments.

Eichler HG, Oye K, Baird LG et al. Adaptive licensing: taking the next step in the evolution of drug approval. Clin. Pharmacol. Ther. 91(3), 426-437 (2012). [CrossRef] [Medline]

31. Senior Medical Officer. Pilot Project on Adaptive Licensing. EMA, UK (2014).

32. Leopold C, Vogler S, Habl C, Mantel-Teeuwisse AK, Espin J. Personalised medicine as a challenge for public pricing and reimbursement authorities - a survey among 27 European countries on the example of trastuzumab. Health Policy 113(3), 313-322 (2013). [CrossRef] [Medline] [CAS]

33. Diseasome. www.diseasome.eu. 



\section{Chapter 3}

\section{An Index of Barriers for the Implementation of Personalised Medicine and Pharmacogenomics in Europe}

Published as: Denis Horgan*, Marleen Jansen*, Lada Leyens*, Jonathan A. Lal, Ralf Sudbrakd Erica Hackenitz, Ulrike Bußhoff, Wolfgang Ballensiefen, Angela Brand (2014). An Index of Barriers for the Implementation of Personalised Medicine and Pharmacogenomics in Europe. Public Health Genomics 2014;17:287-298. DOI: $10.1159 / 000368034$

*Denis Horgan, Marleen Jansen, and Lada Leyens contributed equally in the preparation of the manuscript. 


\section{Abstract}

Background: Personalised medicine (PM) is an innovative way to produce better patient outcomes by using an individualised or stratified approach to disease and treatment rather than a collective treatment approach for patients. De- spite its tangible advantages, the complex process to translate PM into the member states and European healthcare systems has delayed its uptake. The aim of this study is to identify relevant barriers represented by an index to summarise challenging areas for the implementation of PM in Europe. Methods: A systematic literature review was con- ducted, and a gaps-and-needs assessment together with a strengths-weaknesses-opportunities-and-threats analysis were applied to review strategic reports and conduct interviews with key stakeholders. Furthermore, surveys were sent out to representatives of stakeholder groups. The index was constructed based on the priorisation of relevant factors by stakeholders. Results: A need for stakeholder-agreed standards at all levels of implementation of PM exists, from validating biomarkers to definitions of 'informed consent'. The barriers to implement PM are identified in 7 areas, namely, stakeholder involvement, standardisation, interoperable infrastructure, European-level policy making, funding, data and research, and healthcare systems. Conclusions: Challenges in the above-mentioned areas can and must be successfully tackled if we are to create a healthier Europe through PM. In order to create an environment in which PM can thrive for the patients' best outcomes, there is an urgent need for systematic actions to remove as many barriers as possible. 


\section{Preventive Rather than Reactive Healthcare}

The emphasis in modern healthcare has shifted significantly in recent years. Prevention has become one of the hallmarks of healthcare, resulting in preventive rather than reactive healthcare [1-3]. This approach translates into primary prevention of disease, allowing a more accurate diagnosis and specific treatment but also preventing negative individual patient outcomes (e.g. adverse drug reactions) [4]. More attention is paid by pharmaceutical companies as well as healthcare policy makers to combine each person's unique clinical, molecular, and environ- mental information to adjust healthcare to the specific bi- ology of a patient $[2,5]$. Focussing on these individual patient outcomes results in more effective healthcare and greater precision, increasing the quality of care and decreasing healthcare costs $[1,6,7]$. The individualisation of healthcare delivery should be approached by personalised medicine (PM) methodology. PM is defined as a targeted approach to the prevention, diagnosis and treatment of disease based on an individual's specific profile [8].

One of the most common examples of PM is introducing genomics in healthcare, especially as pharmacogenomics (PGx). In PGx the focus is on the predictive outcome of drug interventions [9]. PGx are often companion diagnostics (CDx) for treatment: a molecular assay that, for instance, measures specific mutations to stratify sub- populations, select appropriate medication and tailor dosages to a patient's specific needs [7]. PGx have the potential to change healthcare significantly, since differences between patients' responses to treatment can be partially explained by the genotype (drug metabolism, trans- port and sensitivity) [10]. Using PGx can result in individualised and, consequently, sustainable healthcare, since PGx can increase an effective and safer use of drugs, decreasing costs resulting from drug toxicity and lack of efficacy by identifying patients with the highest probability of therapeutic efficacy [11-14].

CDx will enable PGx to make use of genomic biomarkers: 'a measurable DNA and/or RNA characteristic that is an indicator of normal biologic processes, pathogenic processes and/or response to therapeutic or other interventions' [9]. The identification of biomarkers has significance for individualised intervention regimens, but the process of biomarker discovery, validation and clinical qualification is slow $[15,16]$. The full advantages and the potential of PM can only be achieved when it is used in clinical decision making in an informational, structured framework, but the discrepancy between the ability to sequence a genome and to identify a small amount of relevant genes for translation into clinical practice is a major bottleneck [17-19].

The translation of biomarker research into clinical practice is also challenging, since it affects various areas of healthcare. It involves a wide range of stakeholders and entails tackling numerous barriers for implementation $[4,6,10,13,20]$. In 
order to integrate PM in clinical practice, these barriers must be lifted [1, 2]. Therefore, we must be able to identify existing barriers in Europe. Currently, the uptake of PM in Europe is stagnant, despite its increasingly abundant benefits [4].

To assess the most relevant barriers, an index will be developed. Different sources and viewpoints will be combined in a systematic literature review, strategic reports analysis, and a stakeholder analysis (surveys and inter- views) across several European countries (fig. 1). This index will target patient access to PM and will focus on testing rather than disease prevention, as currently most developments warrant guidelines in the field of PM tests and treatment [15, 21, 22].

\section{Literature Review of Barriers in PM Implementation}

\section{Methods}

A systematic literature review was undertaken to develop an over- view to contextualise the known barriers of access to PM; PubMed, Web of Science and Medline were searched. Search terms were based on terminology used by e.g. the European Alliance for Personalised Medicine and the authors' own experiences in the field and focussed on (synonyms of): 'barriers' and 'personalised medicine' combined with 'care', 'treatment', 'Europe', 'patient', 'ethics', 'biological', 'clinical', 'public health', 'regulatory', 'legislation', and 'commercial'. Only documents in English were included in the review.

Specific attention was given to European strategic reports in the context of a gapsand-needs assessment and strengths, weaknesses, opportunities and threats (SWOT) analysis from the FP7-project PerMed [23]. The aim of gathering these results is to identify relevant fields, organisations, current initiatives, policies, and capacities related to PM, based on an inventory and synthesis of existing relevant information [24]. The data from PerMed were combined with the results from the review of scientific literature, since it offers a broader scope on the knowledge available. Furthermore, PerMed constitutes a network of national funding bodies and governments at the European level not only adding to the viewpoints in this research, but also ensuring impact on future strategic research approaches. 


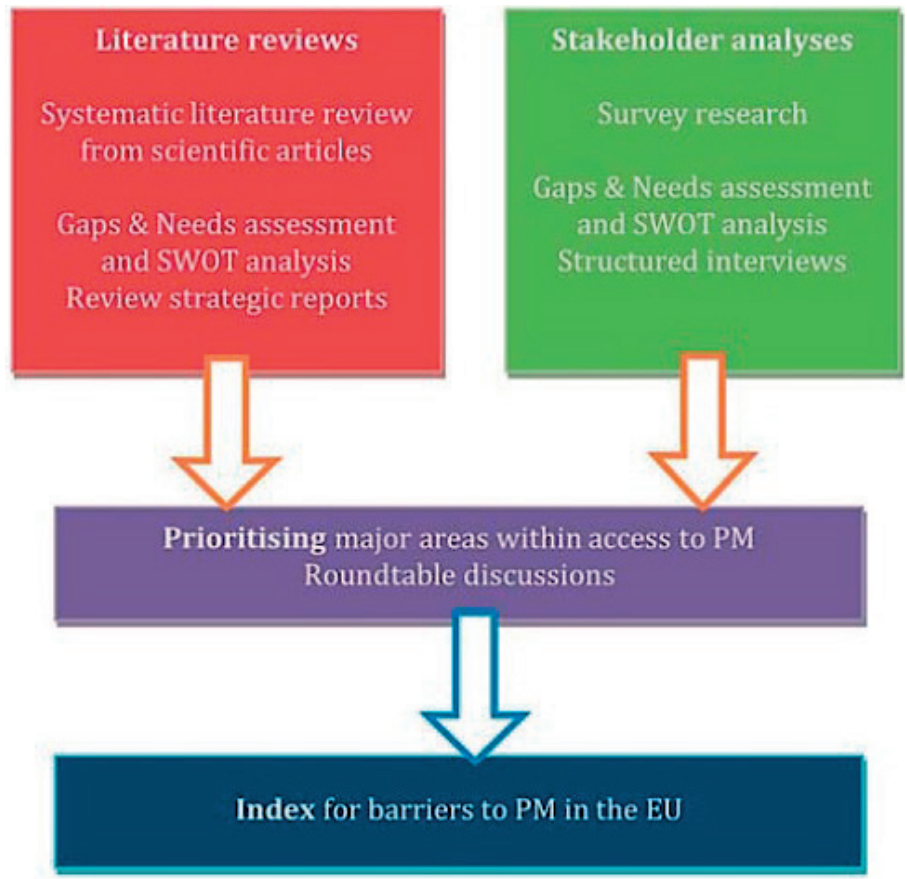

Figure 1. Summary of research methods to develop the index with barriers in access to PM in Europe.

'Gaps' were defined as flaws in the status quo acting as barriers to PM, and 'needs' were considered to be the necessary initiatives to be able to bridge the gaps. A SWOT analysis identifies the factors that shape PM and its enabling characteristics. For the SWOT analysis, the following 4 factors were considered in the reports: (1) helpful to achieve objective, (2) harmful to achieve objective, (3) internal attributes, and (4) external attributes. The relations between these factors are summarised in figure 2.

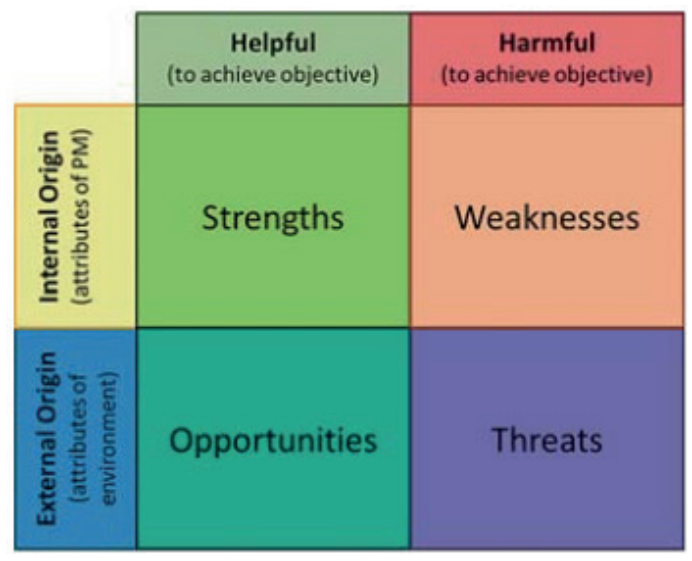

Figure 2. Rationale behind the SWOT analysis [23]. 


\section{Results}

The systematic literature review resulted in 1,543 articles, which were filtered for reviews. Relevance was assessed based on the title and abstract, resulting in 81 articles that were included in the review. The search for strategic reports resulted in 17 papers, gathered from relevant European partners and initiatives in the PerMed network [24]. The strategic reports were assessed on the reported gapsand-needs and analysed for SWOT aspects. The assessment and analysis resulted in an extensive list of gaps-and-needs (185 in total) and information on the SWOT aspects (146 in total) [23].

Striving for PM for patients with the help of healthcare professionals would bring about benefits: better individual patient outcomes, resulting in higher-quality health- care, and a decrease in costs $[3,6,7,16]$. To gain these benefits, the chain leading from research to PM care and treatment needs to overcome barriers. The barriers identified from the analysis were categorised and discussed from (1) scientific, (2) operational, (3) economic, and (4) European levels, as is shown in table 1.

\section{Scientific Level}

On a scientific level, barriers exist, since molecular data is complex to translate into information for clinical practice, for example, because of the heterogeneity of disease subtypes $[25,26]$. In order to provide the information for a valuable implementation of CDx needed in clinical practice, researchers have to attempt to model and understand multiple, interacting and potentially conflicting predictors of risk by integrating several sources of health data [10, 13, 26, 27]. This data is structured in informational models, not only including information on biomarkers and $\mathrm{CDx}$, but also other -omics profiles, clinical data, life- style and environmental data $[5,7,10,19,28]$.

Within these models, CDx can be linked to therapeutics, but on research level, the drug-development process of therapeutics and CDx is not optimised, since several obstacles exist for pharmaceutical and biotechnological companies to develop them simultaneously [7, 29]. Examples that hamper the rapid development of relevant biomarkers are complex regulatory processes for coupled diagnostics, differences in business models, company cultures, and other organisational aspects $[5-7,10,14,30-32]$.

In summary, a lack of consistent evidence exists, which results in clinical uncertainty, for instance, by inadequate validation of biomarkers and inadequate evidence of clinical utility. Development strategies have to be adjusted to clinical needs, to decrease the difficulty to capture the full value of a test generated for clinical practice $[2,6,16,33-39]$. For clinical practice a biomarker test is useful 
when it provides reliable, actionable and predictive information to imply an alternative drug or drug-dosage regimen based on health data in a cost-effective way, as summarized in health technology assessment (HTA) approaches $[4,12,16$, $35,38,40-43]$.

A clinical study design facilitating relevant information gathering for clinical practice is the comparative effectiveness research [2, 7, 44]. This research comprises a direct comparison between 2 approaches of care and their effectiveness in real-world settings, integrates biomarker studies into traditional care, and increases the number of participants in data collection [19]. A new approach to research, however, also implies new approaches to in- formed consent, data collection and storage, for instance, operating with electronic medical records $[19,37]$. These new approaches will need to ensure uniform testing of parameters (including HTA factors), which calls for multidisciplinary guidelines on methodologies $[2,4,13,19,20,25,43]$.

\section{Operational Level}

Not only the operational level within science needs progression to adjust to the PM approach, also other operational levels concerning the translation of PM into healthcare will need to be addressed, i.e. information de- livery, education and training, empowering patients, and regulations (table 1).

Healthcare professionals should be supported by tailored information delivery. An option to facilitate such information delivery is the development of clinical decision support systems (CDSs). Automated CDSs address the lack of infrastructure for delivering treatment and care information and should include genomic and non- genomic health data in order to combine genotypes and standard clinical parameters in an interactive informatics portal, including electronic medical records [18, 25, 27, 30, 42]. Healthcare professionals can catch up with latest scientific findings by continuous dissemination of study results on relevant biomarkers and incorporate risk assessment within disease prognosis and treatment prescription, while preventing an information overload $[3,44,45]$. The information delivery should also be in a timely fashion, i.e. the turnaround time for a biomarker test should be less than the time to make a decision about the course of treatment $[10,40,41]$. 
Table 1. List of summary points from the literature review concerning PM and biomarker testing for CDx, grouped by sections within the levels

\begin{tabular}{|c|c|c|}
\hline Level & Section & Summary points \\
\hline \multirow[t]{3}{*}{ Scientific } & Systems biology and data & $\begin{array}{l}\text { Integrated analyses, health data, biomarkers, informational structured model, drug } \\
\text { development process, HTA framework }\end{array}$ \\
\hline & Research & $\begin{array}{l}\text { Different regulatory processes; business models; company cultures; clinical relevant } \\
\text { information, CER, EMR; informed consent; interoperability }\end{array}$ \\
\hline & Clinical study design & Standards, methodologies, harmonisation; guidelines \\
\hline \multirow[t]{4}{*}{ Operational } & Information delivery & $\begin{array}{l}\text { CDS, infrastructure, limited resources, databases, secure environment, turnaround time } \\
\text { tests }\end{array}$ \\
\hline & Education and training & $\begin{array}{l}\text { Healthcare professionals, lack of knowledge, interpretation, sense of competence, } \\
\text { communication, multidisciplinary team training, curricula medical schools }\end{array}$ \\
\hline & $\begin{array}{l}\text { Inform, educate, empower } \\
\text { patients }\end{array}$ & Low awareness, health literacy, privacy regulations, reduce concerns \\
\hline & Regulations & $\begin{array}{l}\text { Guidelines, lack of consensus, outdated, adherence, standards for practice, clinical } \\
\text { study design, infrastructure of biobanking, ethical and legal issues, automated CDS }\end{array}$ \\
\hline Economic & Reimbursement & $\begin{array}{l}\text { Lack of evidence, incentives, streamline, market-based approaches, HTA, approval } \\
\text { process }\end{array}$ \\
\hline \multirow[t]{4}{*}{ European } & Biobanks & $\begin{array}{l}\text { Lack of standardisation, international interoperability, regulatory procedures, } \\
\text { infrastructure, management, cost investment, EU-funding procedures }\end{array}$ \\
\hline & Translation & Cross-border data collection and sharing, HTA framework \\
\hline & Reimbursement & $\begin{array}{l}\text { Reimbursement models, national healthcare systems, exchanging information, timely } \\
\text { access (patients), market access }\end{array}$ \\
\hline & Legal and ethical & $\begin{array}{l}\text { Sharing confidential data, EU directive, legal framework tests and treatments, privacy, } \\
\text { IP, researchers' accessibility, different legislations, ethics }\end{array}$ \\
\hline
\end{tabular}

Developing and implementing such a system poses a great challenge, since healthcare has limited resources available for implementation, maintenance and sustain- ability [46]. Furthermore, including large patient information databases with electronic medical records re- quires complex infrastructure developments in a real- time and secure environment $[19,31,47,48]$.

Amongst healthcare professionals, overcoming the barrier of lack of knowledge and awareness about CDx would enhance the implementation of PM tests in clinical practice and help to realise the benefits of $\operatorname{CDx}[2,5,10,11,14,17,19,30$, $34,35,41,42]$. Education will need to be provided to healthcare professionals, so increasing their ability and sense of competence in interpreting PM tests and in communicating the results to the patient, since the current lack of knowledge and awareness results in uncertainty about how to interpret a biomarker test or in under-appreciation of the therapeutic benefit $[4,10,19,34,49-52]$. More awareness and knowledge can be gained by supporting healthcare professionals with the above-mentioned CDS but can also be aided by labelling drugs with relevant biomarkers, as already is the case for 121 pharmaceuticals by the Food and Drug Administration in the US [36, 38, 53, 54].

Next-generation healthcare professionals will also need to be educated according to an updated curriculum incorporating PM $[1,3,12,25,55,56]$. The role of information delivery to patients is not limited to medical doc- tors but includes 
also practice nurses and pharmacists, so the education for several healthcare professions will need to incorporate PM using CDx [57-59]. Furthermore, it is important to incorporate education on PM tests in core medical education, since it also imposes challenging ethical implications [5]. Besides education, healthcare professionals should be aware of (genetic) counsellors and their expertise regarding genetic information, and multi- disciplinary team training is suggested $[4,25,39,60]$.

Not only healthcare professionals, but certainly the public and patients have low awareness of the value and integration of biomarkers [2, 3, 38, 53]. In summary, health literacy needs to be increased by clear communication channels to adjust the public's expectations and in- formation about biomarkers (including genomic assays). Besides the utility of $\mathrm{CDx}$, concerns on privacy regulations and genetic discrimination that exist among the public should be reduced [3, 25, 61-65]. Within the communication to patients, the legislation for privacy concerning genomic information has to be stated clearly [2]. Attention should be paid to the relation between the social economic status and health literacy, alongside genomic factors that are specific to minorities and/or could be misused for discrimination between societies $[5,25]$.

In order to provide clear communication to the public and other stakeholders involved, several aspects were named that should be standardised and harmonised at regulatory level (table 1):

1) infrastructure of biobanking (e.g. clinical study de- sign, accessibility of researchers to data, and intellectual property) $[2,19,41,45,66]$,

2) legal and ethical issues (e.g. informed consent, re- imbursement, data safety) $[30,38,41,45,57]$, and

3) automated CDS (e.g. dosing guidelines, interpretations resulting from testing) $[2,13,19]$.

Barriers exist in part due to the lack of adequate application of current regulations but also because of a lack of consensus in guidelines on interpretation and use of PM tests [5,35]. Drug administration authorities should up- date regulatory guidelines with several stakeholders involved in partnerships to provide guidance, clarity and predictability to research and clinical practice, and these should be enforced to increase adherence to guidelines $[4,6,7,19,27,67-69]$.

\section{Economic Level}

Besides the concerns raised on scientific and operational levels, barriers on the economic level are also entangled in the process from research to the implementation of PM. Currently, insurance companies are reluctant to reimburse biomarker tests and treatment because of the lack of evidence and incentives. As a 
consequence, reimbursement is non-existent or insufficient, while it is stated that changing the reimbursement environment could be the most direct way to generate the desired evidence of effectiveness in biomarker research $[2,6,7,17$, $36,70]$.

Biomarker research should support HTA evidence needed for a streamlined reimbursement process by using a market-based approach. An HTA framework can sepa- rate useful from ambiguous CDx and support PM to reach patients in an equitable and transparent fashion $[19,30,34,57,60,71,72]$.

\section{European Level}

As discussed above, barriers exist at the levels of science, operation and economics. These barriers are highly relevant at the European level. Europe has fragmented regulation and approval authorities, which poses problems in bridging the barriers mentioned above [73, 74]. Moreover, realising the promise of cost savings under the PM approach will first require investments at the European level, which is perceived as a considerable barrier [73].

Essentially, coordination and cooperation across Europe is needed on (1) biobanks, (2) translation, (3) reimbursement, and (4) legal and ethical issues (table 1). Several initiatives exist to overcome the gaps in a comprehensive inventory of biobanks and disease registries in Europe, e.g. by the European Medicines Agency, and the European Commission [73, 75]. Besides the general issues illustrated on scientific, operational and economic levels, barriers to harmonisation exist in Europe. Different national governments are involved facilitating health- care to their citizens. This calls for international interoperability, e.g. managing data safety, standardised data collection, international trials, and European funding procedures [74, 76, 77].

Besides differences in the regulations on data collection, the evaluation and translation of biomarker research data varies in Europe across countries [73, 74]. Currently, different methodologies for HTA are used, and appropriate methods concerning comparators should be stated clearly across Europe [3, 74, 76]. Exchanging information between countries can support decision making on reimbursement across borders and ensure timely access for patients to effective PM treatment $[3,74,76]$.

There are clear regulations for pharmaceuticals in the EU; however, there appears to be a lack of a framework for CDx [75]. A partial explanation can be found in the fact that pharmaceuticals and CDx fall under different legislation. These concerns are currently addressed by the European Federation of Pharmaceutical Industries and Associations and the European Medicines Agency [73, 75]. Legislation for medicines, diagnostic regulations, data protection, informed consent, specimens 
use, and medical devices need to be harmonised and must take into account international coherence, since European citizens and data move across borders $[45,77-79]$.

In short, harmonising data in biobanks together with the international interoperability between biobanks can aid research and translation towards effective PM. Such biobanks will offer standardised information on bio- markers for $\mathrm{CDx}$, and by this contribute to the evidence base for HTA. This information will provide input for CDS for healthcare professionals and reimbursement across Europe, within legal frameworks, and will enable a patient-centered and timely implementation of PM.

\section{Stakeholder's Views on Barriers in PM Implementation}

\section{Methods}

Stakeholders were asked about their views on barriers in PM implementation via an online survey and structured interviews. The involved stakeholder groups represented basic science, translational research, the regulatory field, health systems, and patient perspectives.

The data for the online survey was collected by the European Alliance for Personalised Medicine between January and March 2014. Each survey was divided into sections, partially identical among groups and partially tailored to the stakeholder group. In the invitation e-mail, stakeholders received information about the study and the survey was briefly outlined, thus, fully informing the stakeholders.

Each survey consisted of close-ended questions to ensure comparability among the responses. For example, a section on general information included questions about current healthcare applications to facilitate PM. Other sections focussed on crossborder initiatives (e.g. if they are supported by the government). In summary, a range of variables was included to achieve a nuanced exploration of possible barriers.

Thirty-five structured interviews were conducted in February and March 2014 within the PerMed project [23]. Interviewees were selected from the stakeholder groups. Each interview took a maximum of $30 \mathrm{~min}$, and several interviewers carried out the interviews. To ensure comparability, the interviewers were informed beforehand and received an interview guide.

Interviews were conducted and analysed according to statements on gaps-andneeds and SWOT factors. Within each group, statements were coded according to the level of stakeholder they represented. The coding was not limited to one 
viewpoint per statement; when stakeholders from different viewpoints made the same statement about a gap, need or SWOT factor, this statement was coded for multiple viewpoints.

\section{Results}

\section{Surveys}

The survey results showed that the majority of respondents were aware of the term PM. The definition differed somewhat; respondents from basic science $(90 \%)$, translational research (90\%), regulatory field (81\%), and patient groups (73\%) viewed PM as individualised care. Overall, respondents from health systems also defined PM as individualised care; however, specifically oncologists viewed it as a stratification of a certain subpopulation (50\%). Looking at the current state of PM in healthcare, applications are still in the test phase, such as ICT tools focussing on DNA sequencing, bioinformatics and statistical analyses. Stakeholders were not sure if biobanks were used in their region, while they stated that their government pre- dominantly does support sharing of anonymous data. However, current research activities do not focus on pre- competitive biomarker research, since incentives for a PM approach to research were considered unavailable or limited.

This lack of streamlined research into PM also translates into the availability of PM in healthcare. Respondents from translational research and the regulatory field viewed PM to have limited availability in healthcare. Eighty percent of the general healthcare providers answered that there are no programmes in place for PM in healthcare, and also the majority of patient representatives agreed to this statement. If PM programmes were in place, patient representatives considered them to be in oncology.

Besides the limited availability of PM in healthcare, the state of best practice guidelines at different stakeholder levels also posed striking differences. Twothirds of the respondents were not aware of any best practice guide- lines. In the field of oncology, most healthcare professionals were aware of best practice guidelines.

Even though programmes to stimulate the application of PM are currently considered unavailable, responses from 'translational research' and 'regulatory field' showed that (national) ethics committees to evaluate molecular tests are in place, and new legal and/or regulatory frame- works are under preparation. These ideas found resonance in responses from 'healthcare', where plans to pro- mote PM were considered to be at the horizon. Amongst patient representatives, the expectations were more divided; half of them thought PM will eventually be available, while half disagreed. Overall, respondents from patient groups were not sure 
whether health insurance covered PM, but felt it would boost its application. In 'basic science' and 'translational research', a boost is more expected from tools to share data according to centralised guidelines and legislation.

It may be expected, when the availability of best practice guidelines is increased, PM will develop at a higher pace. Only patients' representatives considered healthcare professionals to be not well equipped to implement PM applications. Respondents from translational re- search, the regulatory field and healthcare itself deemed healthcare professionals to be well equipped to implement PM applications.

\section{Interviews - Gaps and Needs}

In the interviews, a gaps-and-needs assessment and a SWOT analysis were conducted. The analysis of the interviews resulted in the identification of 63 gaps and 91 needs. Furthermore, 71 strengths, 73 weaknesses, 79 opportunities, and 73 threats were determined [23].

From the patient perspective, patients should be empowered, and it is advisable to look at complementary issues in stakeholder agendas. Early involvement of patient representatives, to allocate funding to their unmet needs and to ensure access to their own data are essential aspects to be tackled.

Basic science is too far apart from PM, limiting the evidence needed to implement PM. Long-term policies in order to integrate -omics will need to be developed to overcome this gap. A large part of preclinical trials is conducted in a commercial setting, hampering transparent knowledge transfer between basic research amongst companies and towards clinical research. More feedback should be communicated back and forth in order to close the circle of research and plan improvements.

Translational research should be more aware of multi-stakeholder needs and incorporate stakeholders in interdisciplinary research and translation centres (e.g. centres of excellence). The focus should be on sharing interpretations and knowledge management, for example, training healthcare professional in IT and interpretation, instead of focussing only on sharing data in research. New research should be conducted to improve clinical decisions, incorporating suggestions of different stakeholders into an implementation plan.

Regulatory speaking, approval of CDx should become a formal part of drugapproval regulations, so processes direct towards generating evidence to link specific tests and treatment outcomes, including regulation and legislation necessary to facilitate safe data storage and data ownership. These data will need to be shared, to sustain studies and ensure feasible and efficient studies that apply to daily practice and that use harmonised methodology for small populations. 
The way healthcare decision making and healthcare delivery is currently facilitated should be reconsidered to allow a more effective, efficient and timely translation from science to healthcare. The inclusion of PM in the education of healthcare professionals is vital, and more refined information systems are required to support them in translating PM to their patients, hence increasing health literacy. Funding should be allocated to unsolved ethical and legal issues as well as developing an inventory in healthcare systems.

\section{Interviews - SWOT}

PM strengthens efficacy of healthcare by necessitating close collaboration between its stakeholders. These stake- holders will focus on an organised discussion to develop, for instance, guidelines, which will facilitate better rational treatment based on individual health data. Health data that are stored in large datasets directly impacting the interface of patient and healthcare professional will keep clinical practice up-to-speed with science.

'Weakness' can mainly be found in the evidence that is needed to facilitate PM. Not only are disease mechanisms highly complex, individual parameters do not reflect the full story; technologies to store and analyse big data and to try to model them are not fully developed yet. Further- more, the illustrated change in healthcare delivery will be hard to realise in the current rigid system.

Especially in the field of drug development, PM offers opportunities. Cooperation between stakeholders will stimulate innovation, resulting in more successful implementation of CDx combined with a pharmaceutical product prescribed by informed healthcare professionals. Regulations facilitating such targeted treatment will also change the mind-set towards greater efficiency in health- care, not only looking at disease definition critically but also towards innovations targeting public health.

Multi-stakeholder involvement indicated threats to the implementation process, e.g. in the duration of trials and long research processes resulting in patient groups becoming impatient. Not only are these research processes lengthy, they are also costly, and a worst-case scenario would be developing CDx that only increase costs of treatment, but not positive health outcomes. 


\section{Indexing Relevant Barriers in PM}

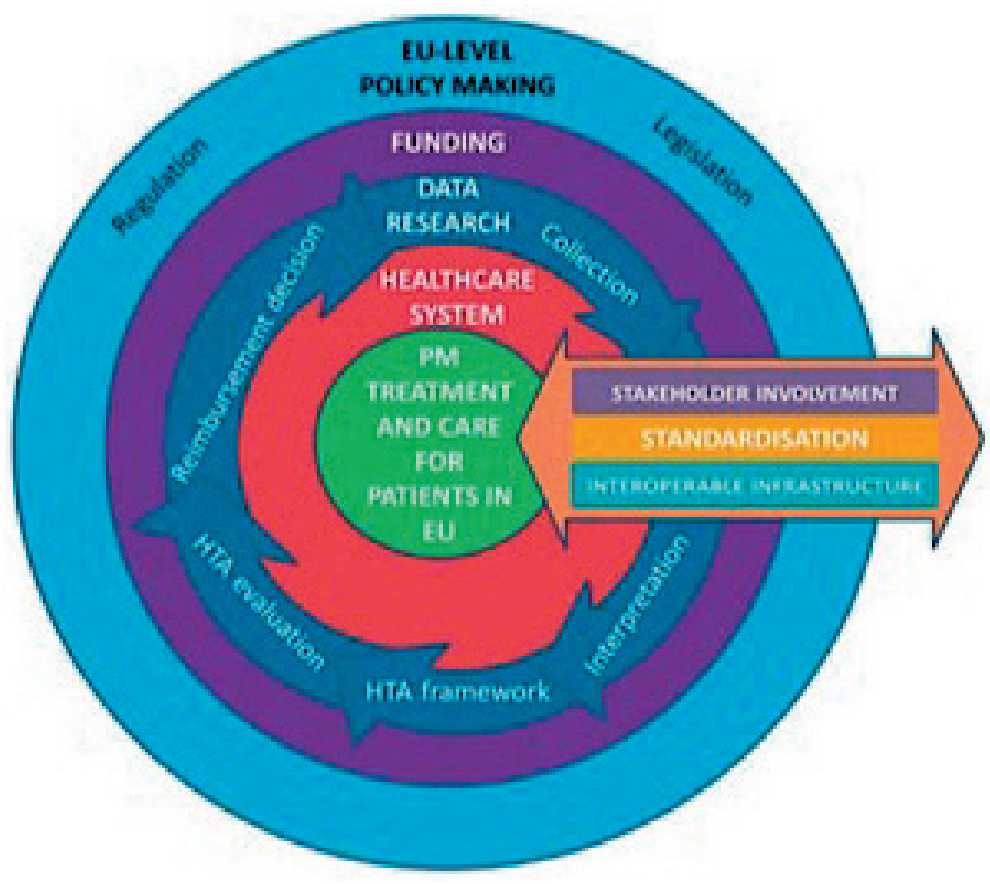

Figure 3. Index of barriers to PM in Europe.

\section{Methods}

After comparing the results from the 2 research arms (literature review and stakeholder analyses) an index was developed. The index consists of relevant barriers to improve access to PM for patients. In order to select relevant barriers, the barriers were scored by representatives of each stakeholder group.

The prioritisation of relevant barriers took place at the PerMed meeting in Berlin on March 28-29, 2014; 82 participants representing different organisations attended the meeting. After a presentation of the identified barriers, each stakeholder group had a session to prioritise the results, both from the strategic reports and the structured interviews.

Each group received the results from the analyses in paper form and was asked to rank 5 major areas to be prioritised regarding the implementation of PM in European health systems. Furthermore, within each of these areas the stakeholders were requested to pin- point 3 specific areas needing attention. The results from these sessions were presented to all participants. The stakeholders' prioritisations in the PerMed meeting were combined with the results from the systematic literature review to select the relevant barriers summarised in the index. 


\section{Results}

Comparing the stakeholders' prioritisations to the summary points from the systematic literature review resulted in the identification of 7 main areas (fig. 3). The barriers facilitating access to PM including to CDx lie mainly in the areas of (1) stakeholder involvement, (2) standardisation, (3) interoperable infrastructure, (4) healthcare system, (5) data and research, (6) funding, and (7) policy making. As an example, the development of 'data and research' is explained below.

In the systematic literature review within the section 'science', the main points concern 'systems biology', 'data and research', and 'clinical study design'. In the prioritisation from the stakeholder groups from the viewpoint of basic science, the 'access to data', 'measurable outcomes', and 'making sense of the data' are considered the most important factors. Furthermore, the stakeholders from translational research and the regulatory field prioritised 'data and research'. All aspects in the literature review and the prioritisation were compared using this approach to define the main areas.

\section{Discussion}

\section{Focus on Harmonisation in Europe}

The goal of this research was to develop an index that shows the most relevant barriers to the implementation of PM and PGx in Europe. In order to develop such an index, 5 approaches were used within 2 main research directions: literature review and stakeholder analysis.

The 5 approaches consisted of (1) a systematic literature review of scientific articles, (2) a gaps-and-needs assessment and SWOT analysis of strategic reports, (3) a survey study among several stakeholders, (4) structured interviews based on gaps-and-needs and SWOTs concerning PM, and (5) the prioritisation of the barriers ac- cording to groups of stakeholders. As mentioned above, comparing the results from these research arms provided an index with 7 main areas: (1) stakeholder involvement, (2) standardisation, (3) interoperable infrastructure, (4) healthcare system, (5) data and research, (6) funding, and (7) EU-level policy making (fig. 3). Different barriers exist in these fields that hamper the access to PM treatment and care for patients across the EU.

The findings from the different research approaches provided comparable data. In order to facilitate PM, the way research is approached and conducted, for example, in the field of drug development, needs revision, e.g. by cooperation between pharmaceutical and diagnostic companies. However, to be able to change research, the chain before and after it also needs to be adapted. The development of best 
practice guidelines is helpful to both direct research and to evaluate results from new re- search.

The harmonisation of gathered information from re- search to implement PM in healthcare is needed to ensure the interoperability between biobanks by providing the same type of data and interpretation. The evaluation and information availability will need to be translated into healthcare by ICT tools to support healthcare professionals in giving information to the patients and also to standardise reimbursement information across the EU. Stake- holder groups need to be involved more in the development and implementation of PM, not only through education and training but also for bottom-up policy making.

Since Europe consists of nearly 30 countries, some specific barriers could be pointed out. These barriers are also relevant in general, but for instance, the interoperability between biobanks is hampered in Europe, since different national frameworks exist, and this increases the complexity of merging datasets. The national differences in legislation and regulations not only impact on interoperability for biobanks but also financial issues such as reimbursement evaluation. Thus, standardised information requirements should be agreed upon.

When comparing the index to survey responses, the barriers that were analysed in this research occur internationally. Most of the stakeholders were not aware of, for example, best practice guidelines for the application of PM, be it on a national or European level. Even though ICT support tools are under development, they are not being adapted at a fast enough pace. However, stakeholders do feel healthcare professionals could be able to implement such care, and healthcare could become more individualised. To facilitate PM research, funding through reimbursement was considered a major boost.

Taking into account the different barriers from this research, the implementation of PM has numerous ones. At the EU level most of them exist on the harmonisation of approaches to research, reimbursement and information delivery. Facilitating such harmonisation, platforms bringing together the stakeholders, should focus on developing standardised legislation and best practice guide- lines for those factors.

The beginning of PM currently seems to be evolving in the field of PGx, and the process of drug development and the study designs can be streamlined to be better adapted to biomarkers and CDx. Starting points to increase access lie in the 7 main areas of the index and the collaboration between different stakeholders to facilitate a safe and effective environment to implement PM.

The recommendations in table 2 are based on this study; however, to provide evidence-based strategies, more research is needed in feasible methods to increase the access to PM for patients, such as implementation and dissemination strategies how to design information de- livery to patients in user-friendly methods. For instance, our study did not show results for the payment strategies if healthcare 
becomes more cross-border because of in- creased collaborations across the EU, or how patient sup- port should be organised when patients use cross-border healthcare. Furthermore, the feasibility and the available resources to achieve the end goal of accessible PM and changing and applying new policies remain unclear and should be assessed in an overview. To facilitate efficient stakeholder involvement in order to harmonise practices throughout the different stakeholder sections within the index undertaking a mapping of the different stakeholders that engage in the process and the different ways that they interact is advisable. Realising the promise of cost savings under the PM approach will first require investment costs at EU level. However, several initiatives exist on stakeholders working together to realise new research approaches in order to facilitate PM by CDx with relevant biomarkers.

\section{Conclusion}

$\mathrm{PM}$ is one of the most innovative areas in the future of health research. At present, its full potential for patients, citizens and the economy in Europe cannot be developed due to fragmented activities, insufficient communication, and lack of generic solutions in the different areas of PM. This study identified the relevant barriers and challenges for the implementation of PM into the health systems across Europe. The derived recommendations to overcome these stumbling blocks will aid policy makers and other key stakeholders to integrate PM into a European health strategy in a timely, effective and efficient manner.

\section{Acknowledgements}

The authors would like to thank the European Alliance for Personalised Medicine (EAPM) and especially their contacts in various organisations, the survey participants, and the record keeping of which is kept with EAPM. More information about the EAPM can be found at http://www.euapm.eu. We would also like to thank PerMed for supporting this important and unique research. The Coordination and Support Action PerMed project (2013-2015) is funded by the EU within the 7th Framework Programme (No. 602139). More information on the project and the partners can be found at http://www.permed2020.eu. 
Table 2. Recommendations based on the barriers within the main areas of the index

\begin{tabular}{|c|c|c|}
\hline & Barrier & Recommendations \\
\hline $\begin{array}{l}\text { Stakeholder } \\
\text { involvernent }\end{array}$ & $\begin{array}{l}\text { - Mismatch in needs and provided information between clinical } \\
\text { practice and research } \\
\text { - Lack of awareness and knowledge on added value of CDx in } \\
\text { clinical practice }\end{array}$ & $\begin{array}{l}\text { Facilitate stakeholder involvement in policy } \\
\text { making and research implementation by } \\
\text { - Early dialogue } \\
\text { - Public private partnerships } \\
\text { - Public debate } \\
\text { - Bottom-up policy making }\end{array}$ \\
\hline Standardisation & $\begin{array}{l}\text { - National focus for research grants } \\
\text { - Lack of comparable data and information in databases } \\
\text { of CDx in healthcare systems }\end{array}$ & $\begin{array}{l}\text { Standardisation of methods across the EU by } \\
\text { - Evaluation research grants } \\
\text { - Guidelines on data collection, } \\
\text { interpretation, and quality assurance } \\
\text { - Information on reimbursement decisions }\end{array}$ \\
\hline $\begin{array}{l}\text { Interoperable } \\
\text { infrastructure }\end{array}$ & $\begin{array}{l}\text { - Lack of ICT-support tools for data and information sharing } \\
\text { Lack of data and information across the EU to support research } \\
\text { with PM approach }\end{array}$ & $\begin{array}{l}\text { Develop ICT tools for data- and information } \\
\text { sharing, which should include information on } \\
\text { - Biobanks } \\
\text { - Data accessibility } \\
\text { - Information on best practice guidelines }\end{array}$ \\
\hline Policy making & - Scattered policies and legislation across the EU & $\begin{array}{l}\text { Update and/or develop regulations and } \\
\text { legislation at EU level, such as } \\
\text { - Best practice guidelines (research, } \\
\text { reimbursement, implementation, CDS) } \\
\text { - Harmonise legislation for diagnostics and } \\
\text { treatment, development processes }\end{array}$ \\
\hline Funding & $\begin{array}{l}\text { - Lack of harmonised funding by limited reimbursement for } \\
\text { biomarkers in CDx }\end{array}$ & $\begin{array}{l}\text { Adjustment of funding models } \\
\text { - Best practice guidelines } \\
\text { - Conditional reimbursement } \\
\text { - Adaptive licensing }\end{array}$ \\
\hline $\begin{array}{l}\text { Data and } \\
\text { research }\end{array}$ & $\begin{array}{l}\text { - Lack of relevant data: clinical practice, HTA indicators } \\
\text { - Lack of information to interpret health data for clinical } \\
\text { practice } \\
\text { - Lack of information relevant for HTA evaluation } \\
\text { - Lack of reimbursement }\end{array}$ & $\begin{array}{l}\text { Point-of-care research (CER) } \\
\text { - Agreements on type of data, annotation } \\
\text { and quality } \\
\text { - Adjusted informed consent (EMR) } \\
\text { Integrated informational models } \\
\text { - Harmonisation of relevant data } \\
\text { - Updated disease models } \\
\text { Use HTA framework in CER: } \\
\text { - Best practice guidelines }\end{array}$ \\
\hline $\begin{array}{l}\text { Healthcare } \\
\text { system }\end{array}$ & $\begin{array}{l}\text { - Lack of awareness and knowledge of CDx before starting } \\
\text { treatment } \\
\text { - Lack of support in clinical decision making } \\
\text { - Lack of uptake of PM }\end{array}$ & $\begin{array}{l}\text { Training and education } \\
\text { - Workshops } \\
\text { - Automated support tools } \\
\text { - Curricula healthcare professionals }\end{array}$ \\
\hline PM to patient & $\begin{array}{l}\text { - Lack of awareness and knowledge value and integration of } \\
\text { biomarkers }\end{array}$ & $\begin{array}{l}\text { Information about PM to patients, e.g. by } \\
\text { - Education programmes by government } \\
\text { - Communication by healthcare } \\
\text { professionals }\end{array}$ \\
\hline
\end{tabular}




\section{References}

1 Abrahams E, Silver M: The case for personalized medicine. J Diabetes Sci Technol 2009;3: 680-684.

2 Chan IS, Ginsburg GS: Personalized medicine: progress and promise. Annu Rev Genomics Hum Genet 2011;12:217-244.

3 iNNOVAHEALTH: Building on open innovation ecosystem in Europe for healthcare. Cyprus EU Presidency. iNNOVAHEALTH Conference, Larnaca, October 2012.

4 Pirmohamed M: Acceptance of biomarker-based tests for application in clinical practice: criteria and obstacles. Clin Pharmacol Ther 2010;88:862-866.

5 Squassina A, Manchia M, Manolopoulos VG, Artac M, Lappa-Manakou C, Karkabouna S, Mitropoulos K, Del Zompo M, Patrinos GP: Realities and expectations of pharmacoge- nomics and personalized medicine: impact of translating genetic knowledge into clinical practice. Pharmacogenomics 2010;11:1149- 1167.

6 Davis JC, Furstenthal L, Desai AA, Norris T, Sutaria S, Fleming E, Ma P: The microeconomics of personalized medicine: today's challenge and tomorrow's promise. Nat Rev Drug Discov 2009;8:279-286.

7 Cohen JP: Overcoming regulatory and eco- nomic challenges facing pharmacogenomics. N Biotechnol 2012;29:751-756.

8 European Alliance for Personalised Medicine. http://euapm.eu/who-we-are/ (accessed March 5, 2014).

9 Burt T, Dhilloon S: Pharmacogenomics in early-phase clinical development. Pharma- cogenomics 2013;14:1085-1097.

10 Johnson JA, Cavallari LH: Pharmacogenetics and cardiovascular disease - implications for personalized medicine. Pharmacol Rev 2013; 65:987-1009.

11 Bakhouche H, Slanař 0: Pharmacogenetics in clinical practice. Prague Med Rep 2012;113: 251261.

12 Scott SA: Personalizing medicine with clinical pharmacogenetics. Genet Med 2011;13:987- 995.

13 Gervasini G, Benítez J, Carrillo JA: Pharmacogenetic testing and therapeutic drug monitoring are complementary tools for optimal individualization of drug therapy. Eur J Clin Pharmacol 2010;66:755-774.

14 Sorich MJ, McKinnon RA: Personalized medicine: potential, barriers and contemporary is- sues. Curr Drug Metab 2012;13:1000-1006.

15 Staratschek-Jox A, Schultze JL: Re-overcoming barriers in translating biomarkers to clin- ical practice. Expert Opin Med Diagn 2010;4: 103-112.

16 Johnson DR, Galanis E: Incorporation of prognostic and predictive factors into glioma clinical trials. Curr Oncol Rep 2013;15:56-63.

17 Deverka PA: Pharmacogenomics, evidence, and the role of payers. Public Health Genom- ics 2009;12:149-157.

18 Mousses S, Kiefer J, Von Hoff D, Trent J: Using biointelligence to search the cancer ge- nome: an epistemological perspective on knowledge recovery strategies to enable pre- cision medical genomics. Oncogene 2008; 27(suppl 2):S58-S66.

19 Fiore L, D'Avolio LW: Detours on the road to personalized medicine: barriers to biomarker validation and implementation. JAMA 2011; 306:1914-1915.

20 West M, Ginsburg GS, Huang AT, Nevins JR: Embracing the complexity of genomic data for personalized medicine. Genome Res 2006; 16:559-566.

21 Khoury MJ, Gwinn M, Ioannidis JPA: The emergence of translational epidemiology: from scientific discovery to population health impact. Am J Epidemiol 2010;172:517-524.

22 Roden DM: Cardiovascular pharmacogenomics: the future of cardiovascular thera- peutics? Can J Cardiol 2013;29:58-66.

23 Personalized Medicine: PerMed - FP 7 Project. Sponsored by the European Commission. 2014.

24 Personalized Medicine (PerMed): http:// www.permed2020.eu/1401.php (accessed March 5, 2014).

25 Chung WK: Implementation of genetics to personalize medicine. Gend Med 2007;4:248-265. 
26 Ely S: Personalized medicine: individualized care of cancer patients. Transl Res 2009;154: 303308.

27 US Food and Drug Administration: Paving the way for Personalised Medicine: FDA's Role in a New Era of Medical Product Devel- opment. 2013. http://www.fda.gov/down- loads/scienceresearch/ specialtopics/personalizedmedicine/ucm372421.pdf.

28 Perez EA: Breast cancer management: opportunities and barriers to an individualized ap- proach. Oncologists 2011;16(suppl 1):20-22.

29 Roden DM: Cardiovascular pharmacogenomics: the future of cardiovascular thera- peutics? Can J Cardiol 2013;29:58-66.

30 Cohen MJ, Ginsburg GS, Abrahams E, Bitter- man H, Karnieli E: Overcoming barriers in the implementation of personalized medicine into clinical practice. Isr Med Assoc J 2013;15: 599-601.

31 Deverka PA, Vernon J, McLeod HL: Economic opportunities and challenges for pharmacogenomics. Annu Rev Phamacol Toxicol 2010;50:423-437.

32 L'Office parlementaire d'évaluation des choix scientifiques et technologiques: Rapport pro- visoire: les progrès de la génétique, vers une médecine de précision? Les enjeux scienti- fiques, technologiques, sociaux et éthiques de la médecine personnalisée. 2014.

33 Nunn AD: Molecular imaging and personal- ized medicine: an uncertain future. Cancer Biother Radiopharm 2007;22:722-739.

34 PricewaterhouseCoopers (PwC), European Hospital and Healthcare Federation (HOPE): Personalized Medicine in European Hospitals. 2011.

35 Babić N: Clinical pharmacogenomics and concept of personalized medicine. J Med Bio- chem 2012;31:281-286.

36 Ieiri I: What are barriers to pharmacogenom- ics (PGx) clinical uptake? Drug Metab Pharmacokinet 2012;27:279.

37 Love D, Stratton E, Stocum M: Best practices for companion diagnostic and therapeutic development: translating between the stake- holders. N Biotechnol 2012;29:689-694.

38 Overby CL, Tarczy-Hornoch P: Personalized medicine: challenges and opportunities for translational bioinformatics. Pers Med 2013; 10:453-462.

39 Weitzel JN, Blazer KR, MacDonald DJ, Culver JO, Offit K: Genetics, genomics and cancer risk assessment: state of the art and future di- rections in the era of personalized medicine. CA Cancer J Clin 2011;61:327-359.

40 Zineh I, Lesko LJ: Pharmacogenetics in medi- cine: barriers, critical factors and a framework for dialogue. Pers Med 2009;6:359-361.

41 Ginsburg GS: Barriers and Solutions to Im- plementing Personalized Medicine (presenta- tion). Duke University, Durham, North Caro- lina, 2012.

42 O'Donnell PH, Bush A, Spitz J, Danahey K, Saner D, Das S, Cox NJ, Ratain MJ: The 1200 patients' project: creating a new medical mod- el system for clinical implementation of pharmacogenomics. Clin Pharmacol Ther 2012; 92:446-449.

43 Patel JN: Application of genotype-guided cancer therapy in solid tumors. Pharmacoge- nomics 2014;15:79-93.

44 Williams MS: The public health genomics translation gap: what we don't have and why it matters. Public Health Genomics 2012;15: 132-138.

45 European Alliance for Personalised Medicine: Innovation and patient access to personalized medicine. Report Irish Presidency Conference, Dublin, March 2013.

46 Ackerman MJ, Filart R, Burgess LP, Lee I, Po- ropatich RK: Developing next-generation telehealth tools and technologies: patients, systems, and data perspectives. Telemed J E Health 2010;16:9395.

47 Coleman H, Ashcraft K: Genelex Corporation. Pharmacogenomics 2008;9:469-475.

48 Pulley JM, Denny JC, Peterson JF, Bernard GR, Vnencak-Jones CL, Ramirez AH, Dela- ney JT, Bowton E, Brothers K, Johnson K, Crawford DC, Schouldcrout J, Masys DR, Dilks HH, Wilke RA, Calyton EW, Shultz E, Laposata M, McPherson J, Jirjis JN, Roden DM: Operational implementation of prospective genotyping for personalized medicine: the design of the Vanderbilt PREDICT Proj- ect. Clin Pharmacol Ther 2012;92:87-95. 
49 Reynolds KS: Achieving the promise of personalized medicine. Clin Pharmacol Ther 2012;92:401405.

50 Bonter K, Desjardins C, Currier N, Pun J, Ashbury F: Personalised medicine in Canada: a survey of adoption and practice in oncology, cardiology and family medicine. BMJ Open 2011;1:e000110.

51 Pellegrini I, Rapti M, Extra JM, Petri-Cal A, Apostolidis T, Ferrero JM, Bachelot T, Viens P, Bertucci F, Julian-Reynier C: Targeted chemotherapy for breast cancer: patients perception of the use of tumor gene profiling approaches to better adapt treatments (in French). Med Sci (Paris) 2012;28(Spec No 1):24-27.

52 European Alliance for Personalised Medi- cine: EAPM: Big Data workshop. 16th Euro- pean Health forum. Gastein, October 2013.

53 Moridani M, Maitland-van der Zee AH, Sasaki H, McKinnon R, Fleckenstein L, Shah VP: AAPS-FIP summary workshop report: Pharmacogenetics in individualized medi- cine: methods, regulatory, and clinical appli- cations. AAPS J 2009;11:214-216.

54 Crews KR, Cross SJ, McCormick JN, Baker DK, Molinelli AR, Mullins R, Relling MV, Hoffman JM: Development and implementa- tion of a pharmacist-managed clinical phar- macogenetics service. Am J Health Syst Pharm 2011;68:143-150.

55 Aspinall MG, Hamermesh RG: Realizing the promise of personalized medicine. Harv Bus Rev 2007;85:108-117.

56 Büro für Technikfolgen-Abschätzung beim Deutschen Bundestag: Individualisierte Me- dizin und Gesundheitssystem. Zukunftsre- port. 2008. http://www.bfa-gemeinschaft.de/ index.php/grund saetze-u-ziele/68-bundestag/soziale-sicherheit/164-zukunftsmedizin-forschung-im-berichindividualisierte- medizin.

57 McKinnon R, Ward MB, Sorich MJ: A critical analysis of barriers to the clinical implemen- tation of pharmacogenomics. Ther Clin Risk Manag 2007;3:751-759.

58 Crews KR, Hicks JK, Pui CH, Relling MV, Ev- ans WE: Pharmacogenomics and individual- ized medicine: translating science into prac- tice. Clin Pharmacol Ther 2012;92:467-475.

59 Chadwell K: Clinical practice on the horizon: personalized medicine. Clin Nurse Spec 2013; 27:3643.

60 European Science Foundation: Personalized medicine for the European citizen. Towards more precise medicine for the diagnosis, treatment and prevention of disease (iPM). 2012. http://www.esf.org/uploads/media/ Personalised_Medicine.pdf.

61 Haddy CA, Ward HM, Angley MT, McKin- non RA: Consumers' views of pharmacoge- netics - a qualitative study. Res Social Adm Pharm 2010;6:221-231.

62 Cornetta K, Brown CG: Balancing personal- ized medicine and personalized care. Acad Med 2013;88:309-313.

63 Shastry BS: Pharmacogenetics and the concept of individualized medicine. Pharmacogenomics J 2006;6:16-21.

66 Ronquillo JG: How the electronic health record will change the future of health care. Yale J Biol Med 2012;85:379-386.

67 Hood L, Friend SH: Predictive, personalized, preventive, participatory (P4) cancer medicine. Nat Rev Clin Oncol 2011;8:184-187.

68 Harvey A, Brand A, Holgate ST, Kristiansen LV, Lehrach H, Palotie A, Prainsack B: The future of technologies for personalised medi- cine. N Biotechnol 2012;29:625-633.

69 Mesko B, Zahuczky G, Nagy L: The triad of success in personalised medicine: pharma- cogenomics, biotechnology and regulatory issues from a Central European perspective. N Biotechnol 2012;29:741-750.

70 Weldon CB, Trosman JR, Gradishar WJ, Ben- son AB 3rd, Schink JC: Barriers to the use of personalized medicine in breast cancer. J On- col Pract 2012;8:e24-e31.

71 Enchin H: Clinician adoption of genetic test- ing for drug metabolizing enzymes: is patient safety the low-hanging fruit of personalized medicine? AMIA Annu Symp Proc 2009; 2009:168-172.

72 Brand A, Lal JA; Public Health Genomics European Network: European Best Practice Guidelines for Quality Assurance, Provision and use of Genome-based Information Tech- nologies: the 2012 Declaration of Rome. Drug Metab Drug Interact 2012;27:177-182. 
73 Gaisser S, Vignola-Gagné E, Hüsing B, En- zing C, van der Valk T: EU policies in personalized medicine-related technologies. Pers Med 2009;6:93-102.

74 Lejeune S, Lacombe D: Towards personalized medicine in the EU: what is needed to facilitate the complex international clinical re- search? Pers Med 2013;10:849-857.

75 Pignatti F, Ehmann F, Hemmings R, Jonsson B, Nueblin M, Papaluca-Amati M, Posch M, Rasi G: Cancer drug development and the evolving regulatory framework for companion diagnostics in the European union. Clin Cancer Res 2014;20:1458-1468.

76 Miller I, Ashton-Chess J, Spolders H, Fert V, Ferrara J, Kroll W, Askaa J, Larcier P, Terry PF, Bruinvels A, Huriez A: Market access challenges in the EU for high medical value diagnostic tests. Pers Med 2011;8:137-148.

77 eHealthTaskForce: Redesign Health in Eu- rope for 2020. European Union 2012.

78 Regniault A, Kupecz A, Gavey M, Mignolet O, De Carlo P, Meyer P, Bailey S: Legal and ethi- cal concerns in personalized medicine: a Eu- ropean perspective. Pers Med 2009;6:517-

64 Butrick M, Roter D, Kaphingst K, Erby LH, Haywood C Jr, Beach MC, Levy HP: Patient reactions to personalized medicine vignettes: 528. an experimental design. Genet Med 2011;13:421-428.

65 Luque JS, Quinn GP, Montel-Ishino FA, Arevalo M, Bynum SA, Noel-Thomas S, Wells KJ, Gwede CK, Meade CD; Tampa Bay Commu- nity Cancer Network Partners: Formative research on perceptions of biobanking: what community members think. J Cancer Educ 2012;27:91-99.

79 Payne K, Annemans L: Reflections on market access for personalized medicine: recommendations for Europe. Value Health 2013; 16(suppl 6):S32-S38. 



\section{Chapter 4}

\section{Available tools to facilitate early patient access to medicines in EU and the USA: analysis of conditional approvals and the implications for personalized medicines}

Published as: Leyens L., Richer E., Melien Ø., Ballensiefen W., Brand A. (2015). Available Tools to Facilitate Early Patient Access to Medicines in the EU and the USA: Analysis of Conditional Approvals and the Implications for Personalized Medicine. Public Health Genomics 2015;18:249-259. (DOI:10.1159/000437137) Editor's Choice -- Free Access 


\section{Chapter 4}

\section{Abstract}

Scientific knowledge and our understanding of the human body and diseases have limited any possible treatment tailoring to each patient. The technological advances enabling the integration of various data sets (e.g. '-omics', microbiome, epigenetics and environmental exposure) have facilitated a greater understanding of the human body, the molecular basis of disease and all the factors influencing disease onset, progression and response to treatment, thereby ushering in the era of personalized medicine. We evaluate the regulatory approaches available to facilitate early patient access to efficacious and safe compounds in the EU and the USA in order to make more informed recommendations in the future as to the gaps in regulations for early patient access. An in-depth analysis of conditional approvals (EU) and accelerated approvals (USA) is performed based on the publicly available information (European public assessment reports and a summary review of products approved under both programmes). The types of product, indications, time to approval and type of evidence submitted were analysed. Between 2007 and early 2015, 17 products were conditionally approved in the EU and 25 in the USA, most of them in the area of oncology and based on evidence from phase II clinical trial data. Early approval of promising products based on data from early phases of development is already possible in the EU and the USA. Some of the improvements could entail implementing a rolling assessment of evidence in Europe and extending the scope of early dialogues. 


\section{Introduction}

Scientific knowledge and our understanding of the human body and diseases limit any possible treatment tailoring to each patient. Doctors have always used precise medicine, i.e. as precise as the knowledge in medicine allowed them to do, and they continue to do so. What is different in the so-called 'new era of personalized medicine' are the great advances in '-omic' sciences (genomics, transcriptomics, proteomics, metabolomics, etc.) and in Information and Communication Technologies in the previous 2 decades, and consequently in our understanding of the molecular mechanisms of diseases. The staggering fall in the costs of gene sequencing and genotyping together with other technological advances enabling the integration of various data sets (e.g. microbiome, epigenetics and environmental exposure) have facilitated a greater understanding of the human body, the molecular basis of disease and all the factors influencing disease onset, progression and response to treatment [1,2].

In drug development, there has been a paradigm shift in the past 30 years. We have moved from an anatomical classification, a 'one-size-fits-all' concept with large disease groups (e.g. all cancers classified under one disease group) and blockbuster medicines that are used to treat millions of patients, through a histological classification of disease to a molecular classification [3] and the stratification into smaller sub-groups of conditions and patients, each treated with different drugs targeted at specific genetic variations or immunological markers. Prediction and prevention, an increased efficacy, improved dosing and improved safety are some of the strengths of personalized medicine.

Some of the recent failures in stratified medicine [5] show the need for a better understanding of the molecular basis of diseases. However, the continuing advances in scientific knowledge will facilitate the move from the current stratified approach, which relies on static biomarkers of disease (with the exception of the new immunotherapies in cancer), to a truly individualized treatment, which considers the combination of dynamic biomarkers, disease heterogeneity in time, the ever changing environment, epigenetics and many other factors that modulate disease and response to treatment.

\section{PerMed Strategic Research and Innovation Agenda}

Under the FP7 programme, the European Commission funded a Coordination and Support Action on Personalized Medicine (PerMed). The main goals of the PerMed consortium were to complement existing activities in Europe by identifying and promoting promising research topics and bringing forward the implementation of personalized medicine for the benefit of society. The main output is the 
development of a Strategic Research and Innovation Agenda (SRIA) for Europe and beyond. The interaction of key European players was essential in this task and made it such a unique project. European and national decision-makers and funding bodies initiated this specific action to promote coordination and cooperation efforts between European key stakeholders showing their strong commitment to personalized medicine.

The methodology for the SRIA has been very rigorous, starting with an in-depth gaps-and-needs analysis of the available reports and literature, complemented by telephone interviews with key stakeholders in the different areas of public health and drug development. This was followed by workshops and conferences to discuss the results of the gaps-and-needs analysis, to facilitate multi-stakeholder discussions and to complete the SRIA. The SRIA was presented at a press conference in Brussels in June 2015 [2].

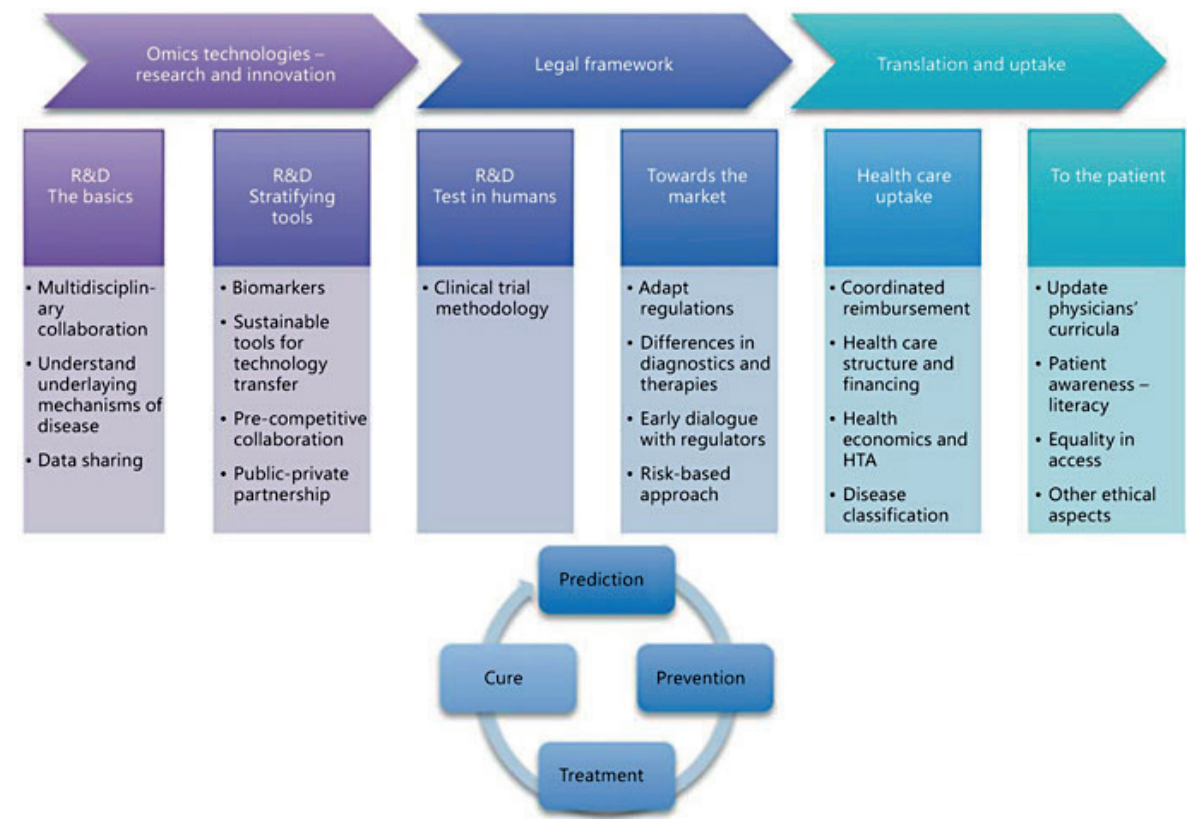

Figure 1 Gaps-and-needs analysis for the personalization of medicine. Adapted framework from the European Commission [1] with the results from 18 reports on personalized medicine [2].

The results of the literature-based gaps-and-needs analysis have previously been published and will not be listed in this paper [4,7]. Figure 1 shows the main areas of concern identified in the analysis. In this paper, we concentrate on the area of the 'legal framework' and, more specifically, on the aspects of systematic early dialogue and early patient access (fig. 1, identified as the risk-based approach); future evaluations will also look at other aspects such as innovative clinical trial methodologies and designs. We evaluated the currently available tools in the EU 
and the USA. Furthermore, we made a systematic and detailed analysis of the conditional approvals (CAs) granted in the EU and the USA [named 'accelerated approvals' (AAs)] and the evidence accepted by regulatory authorities for this riskbased approach to marketing authorization. This analysis elucidates how prepared regulators are to accept early clinical evidence if it shows promising safety and efficacy data, and this can inform future discussions on the need to adapt regulations and approval pathways for personalized medicine.

\section{Early Patient Access/Risk-Based Approaches}

For marketing authorization, the benefit-to-risk ratio of a drug is evaluated. Only drugs with positive benefit-to-risk ratios obtain authorization and are then available to patients to treat the approved indication (or to be used off label at the physician's discretion). In Europe, once marketing authorization is obtained, country-specific criteria are applied to decide whether drugs will be reimbursed within national health care systems and to set their prices; these criteria vary between relative effectiveness analysis (e.g. France, Germany), cost-effectiveness analysis (e.g. England) and other methods.

In the previous 10 years, we have seen the appearance of a number of regulatory tools that aim to facilitate earlier access of patients to efficacious and safe medicines. These tools can be either based on early, iterative and continuous dialogue or on risk-based marketing authorization pathways. By risk-based approaches, we refer to programmes that accept a higher degree of uncertainty if a drug shows very promising early efficacy results and an acceptable safety, leading to the sharing of risks between the regulator (and the general public) and the applicant. We present the different available tools in this section and evaluate the use of the CA process in the EU and the USA during the previous 5 years in detail in the next section. Table 1 and figure 2 present the different tools we identified to facilitate early patient access, differentiated by stage of regulatory evaluation and characteristic of the tool. 
Table 1. Available regulatory tools for early dialogue and early patient access [6,9]. AA= Accelerated Assessment; $\mathrm{CA}=$ Conditional Approval; $\mathrm{CE}=$ Cost-effectiveness; EAMS = Early Access to Medicines Scheme; EMA= European Medicines Agency; FDA= Food and Drug Administration.

\begin{tabular}{llll}
\hline Stage of regulatory evaluation & Characteristic & EMA & FDA \\
\hline $\begin{array}{l}\text { Marketing authorization } \\
\text { (benefit/risk evaluation) }\end{array}$ & $\begin{array}{l}\text { Early and continuous } \\
\text { dialogue }\end{array}$ & Adaptive pathways & $\begin{array}{l}\text { Fast track } \\
\text { Breakthrough therapy designation }\end{array}$ \\
\cline { 2 - 4 } & Faster evaluation & Accelerated assessment & $\begin{array}{l}\text { Fast track } \\
\text { Priority review }\end{array}$ \\
\cline { 2 - 4 } & Less evidence & $\begin{array}{l}\text { CA } \\
\text { Exceptional circumstances }\end{array}$ & AA \\
\hline $\begin{array}{l}\text { Market access } \\
\text { (relative effectiveness/ } \\
\text { cost-effectiveness evaluation) }\end{array}$ & $\begin{array}{l}\text { Early market access } \\
\text { (limited to EU market) }\end{array}$ & $\begin{array}{l}\text { Managed entry agreements } \\
\text { Cancer Drugs Fund (UK) }\end{array}$ & $\begin{array}{l}\text { Payers decisions, rebates and } \\
\text { negotiations (plus Medicare/ } \\
\text { EAMS (UK) }\end{array}$ \\
\hline
\end{tabular}
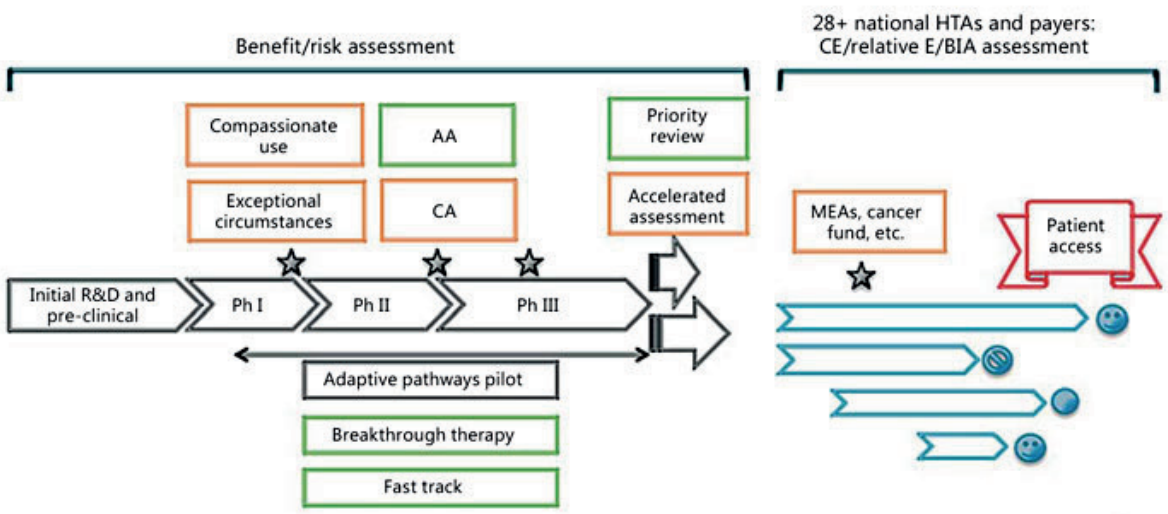

$0-10$ years

Figure 2. Available regulatory tools for early dialogue and early patient access placed on the current drug development phases from initial R\&D to the market. Orange boxes: programmes available in the EU. Green boxes: programmes available in the USA. Colours refer to the online version only. $A A=$ Accelerated Assessment; $\mathrm{CA}=$ Conditional Approval; $\mathrm{CE}=$ Cost-effectiveness; relative $\mathrm{E}=$ relative effectiveness; $\mathrm{BIA}=$ budget impact analysis; $\mathrm{Ph}=$ phase.

\section{Marketing Authorization (Benefit/Risk Evaluation)}

\section{Early and Continuous Dialogue}

In addition to the well-established scientific advice process, whereby applicants approach regulators with specific questions on product development, there are new programmes and pilots available to facilitate a more continuous and iterative dialogue between regulators, applicants and other stakeholders during the whole drug development cycle. In the USA, drugs that treat unmet medical needs, either by providing a therapy for an indication for which there are no other drugs or by improving available therapies, can obtain fast track designation and are eligible for 
more frequent meetings and written communication with the Food and Drug Administration (FDA) to discuss the drug's development programme in addition to the rolling review of evidence. Breakthrough therapy designation is given to drugs that, based on preliminary clinical evidence, demonstrate substantial improvements over available therapies on clinically significant endpoints. The advantages are an intensive guidance to ensure an efficient drug development and an organizational commitment involving senior managers, in addition to all the advantages of the fast track designation. In Europe, the adaptive pathways pilot, launched by the European Medicines Agency (EMA) in 2014, also aims to offer a continuous dialogue from the early phases of development up to marketing authorization for therapies that fill an unmet medical need in order to encourage their efficient development, identify the best marketing authorization application (MAA) pathway and facilitate early patient access. The early involvement of health technology assessments (HTAs) and other stakeholders (including patients) is another important feature of this pilot.

\section{Faster Evaluation Process Leading to Shorter Assessment Periods}

Faster review periods aim to translate into faster marketing authorization and faster patient access. The FDA priority review programme decreases the standard review time by 4 months (from 10 to 6), whereas the European accelerated assessment aims for a 2-month reduction (from the usual 210 evaluation days to 150 days -excluding clock stops). We can also consider the FDA fast track designation under this category, since it offers the possibility of a rolling review of evidence instead of waiting for submission until the entire body of evidence is collected. Such a rolling process can potentially reduce the review time needed to process the full application. To incorporate this into the European regulatory landscape could prove challenging, since all national agencies are involved in the review of centralized authorization procedures.

When it comes to extensions of indication for one single agent, the evidence for its safety can in most cases be comparable to that for the existing indication (if the target population does not dramatically change and the inherent co-morbidities are not very different). Consequently, the review of MAAs should be considerably shorter. One issue that could be considered in Europe is to introduce a mechanism that allows the submission of extensions of indications and new evidence during the review process of the initial MAA; this is possible in the USA and brings with it a considerable delay in the availability of drugs to patients for new indications (we acknowledge, however, that off-label applications are possible after the initial marketing authorization). 


\section{Less Evidence (Risk-Based Approach regarding Evidence Requirements)}

The early approval of medicines based on surrogate endpoints and less stringent evidence requirements has already been granted for a decade for drugs that offer very promising early results. These approvals are conditional on specific postapproval requirements to confirm the initial benefit/risk evaluation, including the need to do additional clinical trials or the finalization of ongoing trials. We will discuss the CA (EU) and AA (USA) processes in more detail in the next section and analyse their use during the previous 5 years in the USA and the EU, the indications for which they were granted, the time to approval and the level of evidence required.

\section{Market Access (Relative Effectiveness/ Cost-Effectiveness Evaluation)}

\section{Early Market Access after Marketing Authorization Has Been Granted (EU Specific)}

The schemes offer early market access and are mostly country and product specific. They facilitate patient access before relative effectiveness, costeffectiveness or other criteria have been established. Managed entry agreements can be based on volume, prices or the results obtained by the medicine; some examples are risk-sharing agreements (used in France and Italy), payment by result (applied in Italy and Spain) or coverage with evidence development (used in the Netherlands and Sweden) [8]. They are usually individually negotiated between companies and authorities and are not transparent; furthermore, their benefits have not been systematically evaluated. The UK has just launched their new Early Access to Medicines Scheme (EAMS), whereby drugs can be made available to patients in the UK in advance of their eventual EU approval. The EAMS involves a two-stage procedure; the first stage involves gaining a promising innovative medicine designation, and the second stage involves gaining a positive scientific opinion from the Medicines and Healthcare Products Regulatory Agency (MHRA). The EAMS also encourages early parallel scientific advice from the MHRA and the National Institute for Health and Clinical Excellence, analogous to the joint scientific advice offered by the EMA and HTAs.

\section{CAs from the EMA and AAs from the FDA}

CAs in the EU and AAs in the USA (table 2) are risk-sharing tools available to applicants to offer early patient access to innovative drugs if the applicants can prove with limited evidence from the early development phases (usually through surrogate endpoints) that their products are of major public health interest and fill an unmet medical need for serious conditions. If this is the case, it may be acceptable to have higher degrees of uncertainty in safety and efficacy and to grant marketing authorization based on post-approval conditions that confirm the initial, 
promising data. The EMA first published their guideline on conditional marketing authorization at the end of 2006; the FDA initiated their programme in 1992, but it was not incorporated into state regulation until it was passed by the Congress in 2012 (FDA Safety and Innovation Act) [9].

Table 2. Definitions of AA (FDA) [10] and CA (EMA) [11]

\section{AA programme}

Early approval based on markers that predict a reasonable benefit, with more testing to confirm a clinical benefit after approval. Allows early approval of a drug for a serious or life-threatening illness that offers a benefit over current treatments. This approval is based on a 'surrogate endpoint' (e.g. a laboratory measure) or other clinical measure the FDA considers reasonably likely to predict a clinical benefit of the drug. Once AA is granted, the drug must undergo additional testing to confirm that benefit; this speeds the availability of the drug to patients who need it.

\section{$C A$}

For certain categories of medicinal products, in order to meet unmet medical needs of patients and in the interest of public health it may be necessary to grant marketing authorizations on the basis of less complete data than is normally required. In such cases, a marketing authorization may be granted subject to certain specific obligations to be reviewed annually. This may apply to medicinal products for human use that belong to one of the following categories:

- medicinal products for seriously debilitating diseases or life-threatening diseases;

- medicinal products to be used in emergency situations, in response to public threats, and - orphan medicinal products (drugs approved for small populations of patients with rare diseases).

An in-depth analysis of the CAs granted by the EMA and the FDA (AA programme) is presented in this section. We have evaluated the CAs and AAs granted during the previous 8 years (2007-2015), and in more detail during the previous 5 years (2011-2015), based on public information published on the EMA and FDA websites. We analysed European public assessment reports (EMA) and summaries of reviews (FDA) to identify the products, the approved indications, the time to approval and the evidence submitted $[12,13,14,15,16]$. We only considered initial MAAs and did not look at further modifications, extensions or submissions for the 
same product; furthermore, in Europe only products approved through the centralized procedures were considered.

Between 2007 and the first half of 2015, 17 products received CA in the EU and 25 drugs received AA in the USA. As depicted in figure 3, most of the approvals were in the area of oncology, followed by anti-infectives and very few drugs for diseases of the nervous system, inborn genetic disorders (mostly enzyme replacement therapies) and ocular conditions (in the EU). There are only 4 medicines in common between the conditionally approved products in the EU and the USA (Zykadia, Sirturo, Adcetris and Xalkori); this may be due to differences in dates of submission to the EMA and the FDA: if submission to one of the two agencies is delayed by the applicant, more mature data may be available, offering the possibility of receiving full marketing authorization. Only $57 \%$ of the drugs approved in the EU had an orphan designation, compared to 95\% in the USA. This may be due to the differences in the definition of orphan drugs (prevalence as defined in the EU is $\leq 5$ in 10,000 compared to $<200,000$ in USA) and the requirements for obtaining the orphan drug designation, or to the small overlap of compounds that obtained conditional approvals between both regions.
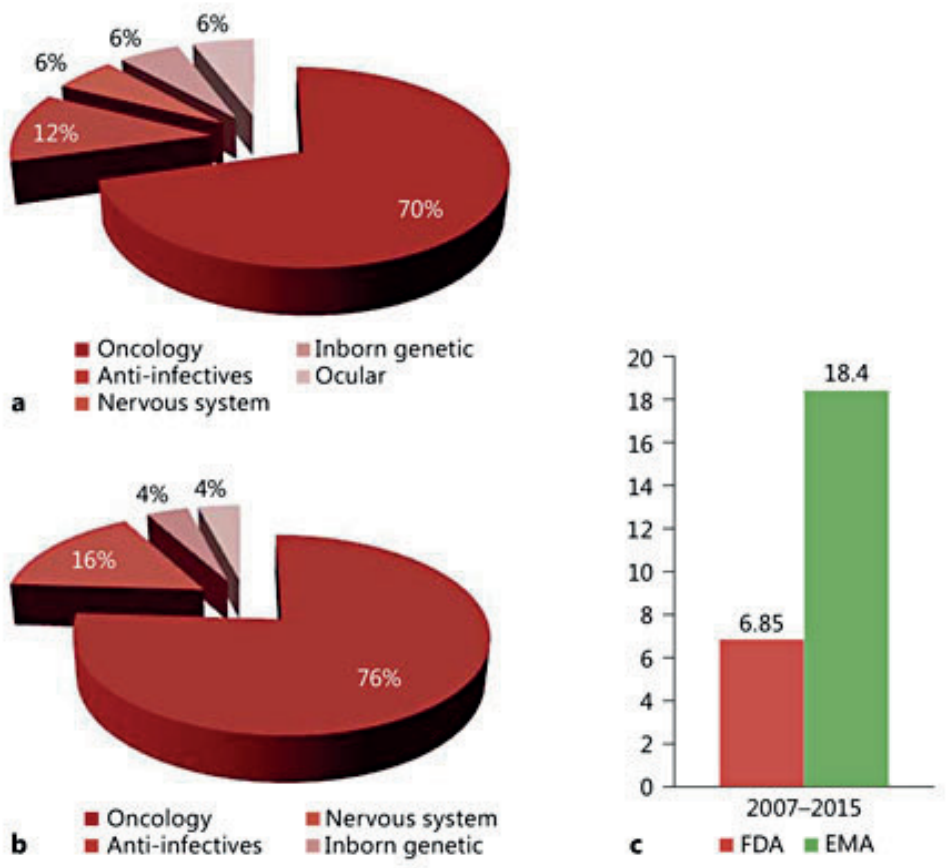

Figure 3. a EMA CAs per indication between 2007 and 2015 ( $\mathrm{n}=17$ ). b FDA AAs per indication between 2007 and $2015(n=25)$. c Median evaluation time (months). 
We will now concentrate on the type of evidence submitted to support marketing authorization accepted by the EMA and the FDA as being sufficient for CA and AA, respectively. Drugs approved during the 2011-2015 period were considered, i.e. 14 drugs in the EU and 20 in the USA. The distribution of products per indication does not significantly differ from that presented in figure 3 , except that the percentage of FDA AAs for oncology medicines increased to $84 \%$.

While the highest proportion of the medicines evaluated was approved based on pivotal data from phase II clinical trials (50\% in the EU and 55\% in the USA), retrospective data analysis was accepted as evidence for 1 drug in each region. These pivotal clinical trials were mostly uncontrolled single-arm trials $(43 \%$ in the EU and 55\% in the USA), followed by randomized placebo-controlled blinded clinical trials (fig. 4).

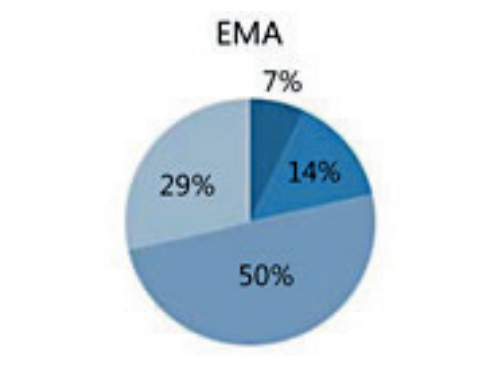

a - Retrospective $=\mathrm{I} / \mathrm{II}=\mathrm{II}=\mathrm{III}$

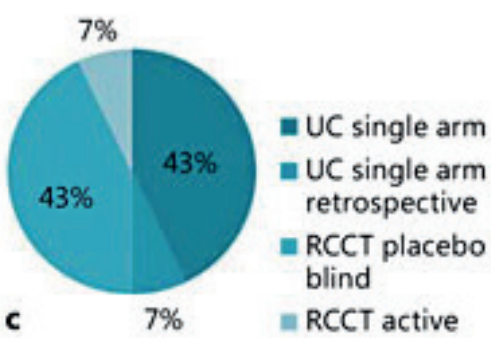

FDA

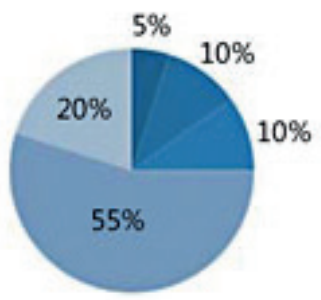

b $=$ Retrospective $=\mathrm{I} \equiv \mathrm{I} / \mathrm{II}=\mathrm{II}=\mathrm{III}$

Figure 4. a EMA CAs. Submitted evidence by phase (I-III) of pivotal CT 2011-2015. b FDA AAs. Submitted evidence by phase (I-III) of pivotal CT 2011-2015. c EMA CAs. Submitted evidence by design of pivotal CT 2011-2015. d FDA AAs. Submitted evidence by design of pivotal CT 2011-2015. a, c n = 14 . $\mathrm{b}, \mathrm{d} \mathrm{n}=20 . \mathrm{CT}=$ Clinical trial; $\mathrm{OL}=$ open label; RRCT $=$ randomized controlled clinical trial; $\mathrm{UC}=$ uncontrolled.

Most products in the USA were approved based on data from 1 or 2 clinical trials, whereas in the EU all products had data from at least 2 clinical trials. Another remarkable difference is the fact that all pivotal trials for the EU-approved products were at least phase II, while in the USA 2 products were approved based on data from phase I trials. The number of trials in figure 5 may be inflated for 
some products due to the fact that extension studies were considered equal to other clinical trials. The number of patients treated in clinical trials with the approved product for the approved indication also varied; most submissions included data from between 100 and 400 patients. Interestingly, some approvals were based on evidence from fewer than 100 patients, which is indeed expected for orphan drugs. The median number of patients was 245 (range 52-588) for EMA-approved products and 179 (range 84-456) for FDA-approved products.
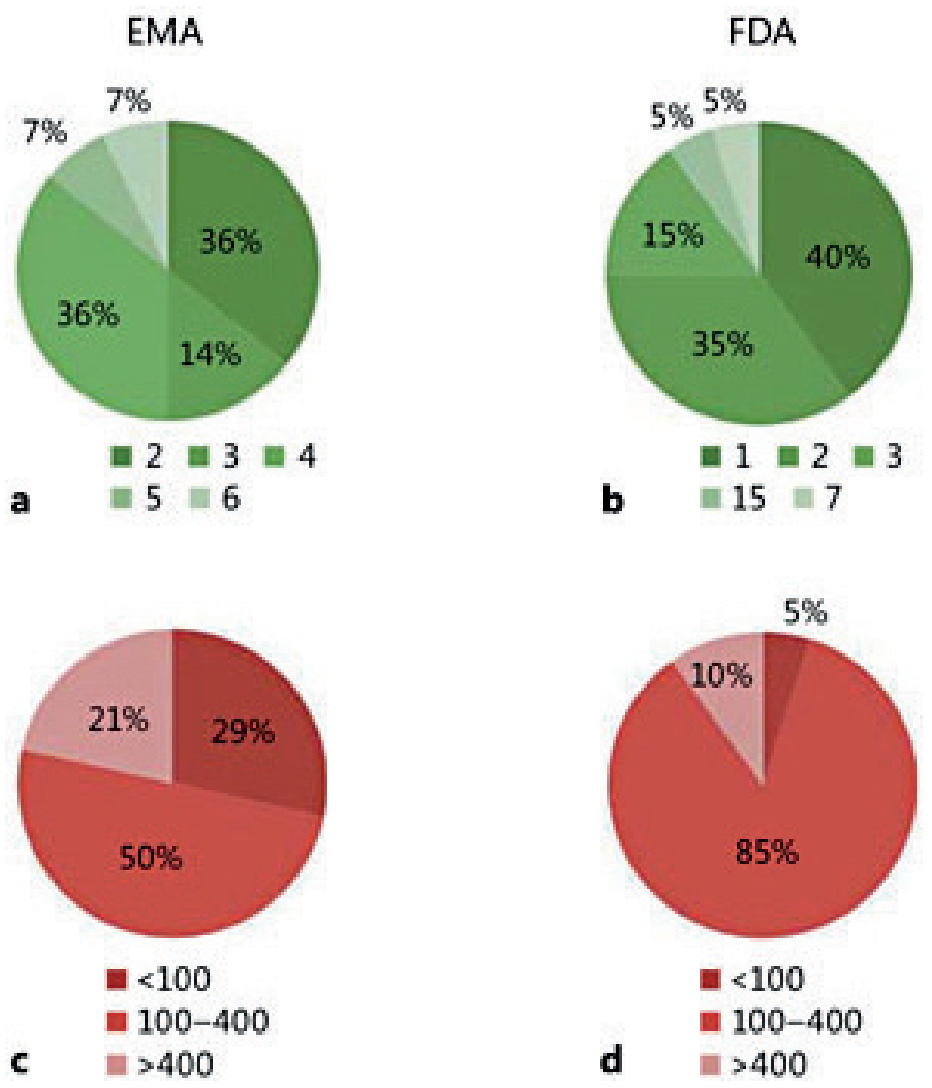

Figure 5. a EMA CAs. Submitted evidence by number of trials 2011-2015. b FDA AAs. Submitted evidence by number of trials 2011-2015. c EMA CAs. Submitted evidence by number of patients 20112015. d FDA AAs. Submitted evidence by number of patients 2011-2015. a, c n =14. b, d n = 20 .

Surprisingly, more than $50 \%$ of the products (50\% in the EU and $70 \%$ in the USA) were approved based on data from uncontrolled clinical trials. Efficacy and safety data from uncontrolled clinical trials represent higher degrees of uncertainty. In the controlled trials, placebo was the most common comparator, with very few products presenting comparisons with active comparators (fig. 6; 1 in the EU and 2 in the USA). FDA-approved products were all based on surrogate endpoints, whereas in the EU, only $64 \%$ of the products had efficacy evidence based on 
surrogate endpoints (they mostly correspond to oncology products, where mature overall survival data can take a long time to collect).
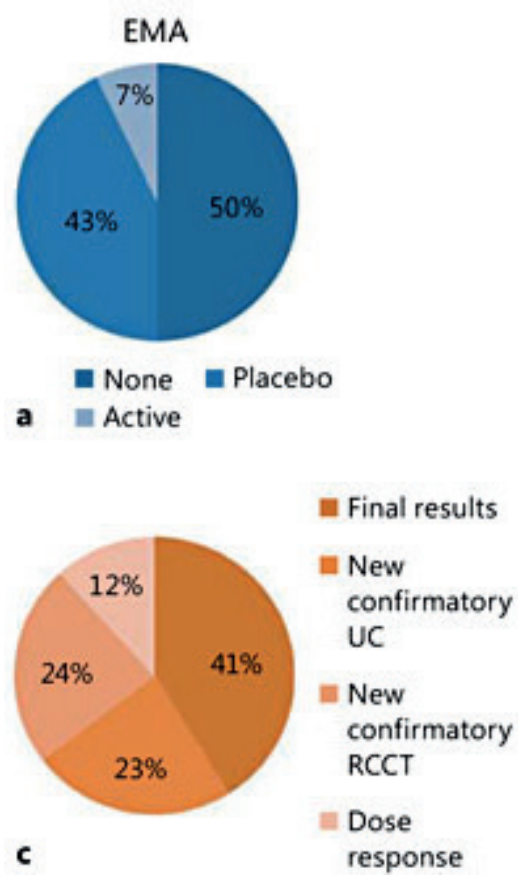
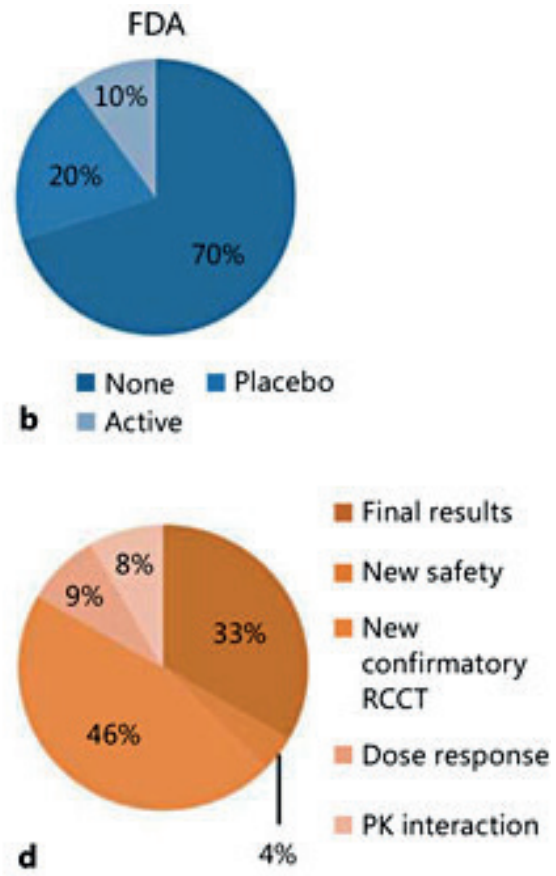

Figure 6. a EMA CAs. Submitted evidence by comparator type 2011-2015 ( $\mathrm{n}=14$ ). b FDA AAs. Submitted evidence by comparator type 2011-2015 $(n=20)$. c EMA CAs. Post-authorization requirements 2011-2015 ( $\mathrm{n}=17$; more than 1 category was requested for 3 products). d FDA AAs. Post-authorization requirements 2011-2015 ( $\mathrm{n}=24$; more than 1 category was requested for 4 products).

In terms of post-authorization requirements, completion of ongoing trials and reporting of final mature results were the most common requirements for the EMA-approved products, whereas the FDA more often requested new confirmatory randomized controlled clinical trials (fig. 6). Other requirements ranged from phase I trials to determine the correct dose and provide doseresponse data, over pharmacokinetic interaction studies to new safety and uncontrolled clinical confirmation trials. We could not identify any trends in the post-authorization requirements that indicated a relationship between uncontrolled evidence and demands for new confirmatory controlled clinical trials; this may be due to the difficulty of conducting controlled trials in some orphan conditions. 


\section{Implications for Personalized Medicine and a Forward Look}

In the previous section, we have seen how approval of medicines based on more limited evidence is possible when promising early results are available and there is a significant unmet medical need. Many experts in the field of personalized medicine are advocating for smaller and fewer clinical trials to speed up the development cycle and allow earlier patient access, and to some extent this is already the case. The high numbers of orphan and oncology drugs in our analysis support the view that smaller subsets of populations and smaller subsets of individual diseases are being characterized that previously have been considered as the same condition (e.g. we now know that the same mutation in tumours from different organs may bear more similarity than two tumours in the same organ with different mutations). As we move into smaller subsets of disease, more conditions could be associated with the 'orphan' definition. Already available drug development strategies for orphan conditions will be used more often; however, the topic of pricing has to be carefully re-evaluated if we are to move into a new classification of diseases for many 'orphan' conditions, since current pricing tactics for orphan drugs would not be sustainable. Alternatively, as we move forward with new precision medicine being introduced, the expected impact in terms of unnecessary drug spending and reduced occurrences of drug adverse events should be introduced into the post-authorization assessment.

It is important to acknowledge that regulators in joint action with HTA bodies have to find the right balance between patients' need to access drugs faster and their inherent mission to protect patients from unsafe/inefficacious drugs. Therefore, a sufficient minimum amount of evidence is needed to ensure that these drugs are indeed safe and efficacious, and a certain time is needed to review the applications and clarify uncertainties with the applicants. Risk-based approaches to marketing authorization aim to share the risk of uncertainties in the benefit-to-risk ratio between applicants and regulators. In most cases, additional data are needed in order to confirm the preliminary conclusions on positive benefits and low risks.

The caveats of this analysis and proposals for future research will be presented here. To start with, only drugs were evaluated; however, different technologies (including medical devices) are used in medical practice and are becoming essential for personalized medicine, and differences in responsible regulatory authorities and regulations for drugs and medical devices in Europe pose additional challenges in this area that are outside the scope of this paper. Furthermore, only initial MAAs were considered; it could be interesting to evaluate further variations as well as the renewal of CAs and AAs, and whether the data submitted within the post-marketing requirements confirmed the initial results. There are some additional schemes facilitating early patient access that have not been discussed here, such as named patient access or compassionate use 
programmes for drugs in development. These can be linked to clinical trials, and physicians have to request special permission from regulatory authorities as well as the developer company for each individual patient. These schemes were excluded because they are not designed to reach the broader patient population and focus on individual patients. There may also be additional country-specific schemes that were not discussed here and could be evaluated in future analyses.

One issue, especially in European markets, is whether HTA bodies and other entities in charge of pricing and reimbursement would also accept the early and immature but promising evidence submitted for CA. HTAs may be hesitant to accept surrogate endpoints and immature data and could request additional data collection, delaying the time to market. In such cases, CAs and other risk-based approaches pioneered by regulators may not result in the earlier patient access aimed at if the bottleneck to market is at the latter stage of market access. However, the emerging developments in the direction of an improved coordination and alignment of regulatory processes and HTAs may provide a future framework for handling these challenges. This issue has to be thoroughly considered and discussed to find solutions able to balance earlier patient access with sufficient evidence for a therapeutic value with reference to clinical effect and safety. To approach these challenges, it appears relevant to take into consideration the use of appropriate tools for monitoring novel pharmacological agents under real-world conditions.

Figure 7 presents the pathway from bench to market (analogous to fig. 2) with some modifications to incorporate a scenario where the tools described in this paper are widely used and early dialogue is the norm. The incorporation of observational studies parallel to later stages of clinical development (as the adaptive pathways pilot aims to promote) to inform final marketing authorization after CAs - or the establishment of a rolling assessment of evidence and a compulsory early and continuous dialogue for promising drugs in the EU (as in the FDA) - would be some of the possible ways to improve the system. 


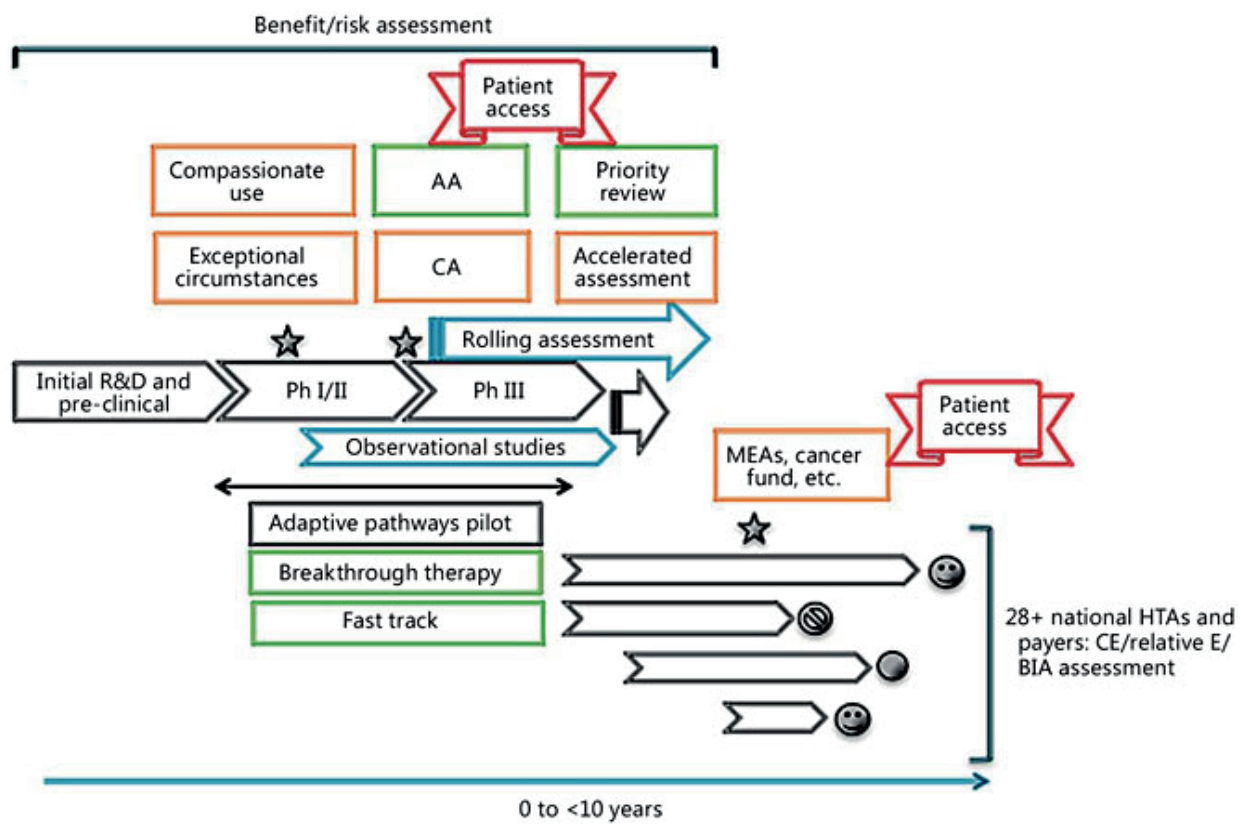

Figure 7. Available regulatory tools for early dialogue and early patient access placed on the revised drug development phases from initial R\&D to the market. Orange boxes: programmes available in the EU. Green boxes: programmes available in the USA. Blue boxes: possible improvements. Colours refer to the online version only. $\mathrm{CE}=$ Cost-effectiveness; relative $\mathrm{E}=$ relative effectiveness; $\mathrm{BIA}=$ budget impact analysis; MEAs = managed entry agreements; $\mathrm{Ph}=$ phase.

From the presented analysis and the regulatory tools for facilitating patient access, we can infer that regulators are indeed starting to accept early clinical evidence if it shows a promising safety and efficacy (as early as in phase I data). Some of the tools may not be used as much as they should, since most of them are voluntary and have to be requested by the applicant. Some of the tools and programmes described may need more refinement, or to be replaced by more appropriate ones, whereas others still need to be evaluated by the regulator (e.g. adaptive pathways pilot). Before strongly arguing for a complete change in regulations, we need to analyse what is available and where it could be improved. Furthermore, change can only happen step-wise; drug development and approval is an ongoing process, with many products in the pipeline that cannot be changed in a very short time span. Finally, and most importantly, we should remember that most regulations in this area had followed huge public health disasters jeopardizing the health of thousands of people (e.g. thalidomide), and, indeed, patients' safety and well-being should be in the foreground in all stakeholders' agendas. 


\section{References}

1. European Commission: Use of '-omics' technologies in the development of personalised medicine. Brussels, European Commission, 2013.

2. PerMed: Shaping Europe's Vision for Personalised Medicine. Strategic Research and Innovation Agenda (SRIA). Cologne, German Aerospace Center (DLR), 2015. http://www.permed2020.eu/ _media/PerMed_SRIA.pdf.

3. Munoz J, Kurzrock R: Targeted therapy in rare cancers - adopting the orphans. Nat Rev Clin Oncol 2012;9:631-642. External Resources

4. Leyens L, et al: Working towards personalization in medicine: main obstacles to reaching this vision from today's perspective. Pers Med 2014;11:641-649. External Resources

5. Berndt ER, Trusheim MR: The segmentation of therapeutic populations in oncology. Health Manage Policy Innovation 2012;1:19-34.

6. European Medicines Agency. http://www.ema.europa.eu/ema/ (accessed June 15, 2015).

7. Horgan D, Jansen M, Leyens L, et al: An index of barriers for the implementation of personalised medicine and pharmacogenomics in Europe. Public Health Genomics 2014;17:287-298. External Resources

8. Ferrario A, Kanavos P: Managed entry agreements for pharmaceuticals: the European experience. Brussels, EmiNET, 2013.

9. US Food and Drug Administration: Fast track, breakthrough therapy, accelerated approval, priority review. http://www.fda.gov/ForPatients/Approvals/Fast/default.htm (accessed June 15, 2015).

10. US Food and Drug Administration: New drugs at FDA: CDER's new molecular entities and new therapeutic biological products. http://www.fda.gov/Drugs/DevelopmentApprovalProcess/ DrugInnovation/default.htm.

11. European Medicines Agency, Committee for Medicinal Products for Human Use: EMEA/509951/ 2006: guideline on the conditional marketing authorisation for medicinal products for human use. London, European Medicines Agency, 2006.

12. European Medicines Agency: European public assessment reports. http://www.ema.europa.eu/ ema/index.jsp?curl=pages/medicines/landing/epar_search.jsp\&mid=WC0b01 ac058001d124 (accessed June 15, 2015).

13. US Food and Drug Administration, Center for Drug Evaluation and Research: Novel New Drugs 2011. Washington, US Food and Drug Administration, Center for Drug Evaluation and Research, 2012.

14. US Food and Drug Administration, Center for Drug Evaluation and Research: Novel New Drugs 2012, Summary. Washington, US Food and Drug Administration, Center for Drug Evaluation and Research, 2013.

15. US Food and Drug Administration, Center for Drug Evaluation and Research: Novel New Drugs 2013, Summary. Washington, US Food and Drug Administration, Center for Drug Evaluation and Research, 2014.

16. US Food and Drug Administration, Center for Drug Evaluation and Research: Novel New Drugs 2014, Summary. Washington, US Food and Drug Administration, Center for Drug Evaluation and Research, 2015. 



\section{Chapter 5}

\section{Early patient access to medicines: Health Technology Assessment Bodies need to catch up with new Marketing Authorization methods}

Published as: Leyens L., Brand A. (2016). Early patient access to medicines: Health Technology Assessment Bodies need to catch up with new Marketing Authorization methods. Public Health Genomics 2016;19:187-191 (DOI:10.1159/000446537) 


\section{Chapter 5}

\section{Abstract}

National and international medicines agencies have developed innovative methods to expedite promising new medicines to the market and facilitate early patient access. Some of these approval pathways are the conditional approval and the adaptive pathways by the European Medicines Agency (EMA); the Promising Innovative Medicine (PIM) designation and the Early Access to Medicines Scheme (EAMS) by the Medicines and Healthcare Products Regulatory Agency (MHRA), as well as the Fast Track, Breakthrough or Accelerated Approval methods by the Food and Drug Administration (FDA). However, at least in Europe, these methods cannot achieve the goal of improving timely access for patients to new medicines on their own; the reimbursement process also has to become adaptive and flexible. In the past 2 years, the effective access (national patient access) to newly approved oncology drugs ranged from 1 to 30 months, with an extremely high variability between European countries. The goal of early patient access in Europe can only be achieved if the national health technology assessment bodies, such as NICE (ENG), HAS (FR), G-BA (DE) or AIFA (IT), provide harmonized, transparent, flexible, conditional and adaptive methods that adopt the level of evidence accepted by the medicines agencies. The efforts from medicines agencies are welcome but will be in vain if health technology assessments do not follow with similar initiatives, and the European 'postcode' lottery will continue. 
New Approaches to Marketing Authorizations of Medicines

\section{Status Quo}

Until recently, the development of medicines had a very rigid and fixed structure: safety and the first proofs of concept were tested in preclinical trials; if the results were promising, the first tests in humans started with phase I clinical trials, followed by clinical trial phases II and III that tested the medicine's efficacy and safety in the appropriate patient population. We used to talk about a 10-year development process, costing around USD 1 billion where only $1 \%$ of medicines initially tested made it to the market [1]. Thanks to the latest developments in science and technology, we understand diseases better and are able to design more targeted drugs, shortening the development process and avoiding the massive fallout of drug candidates on the way to the market. Moreover, new simulation mechanisms and clinical trial designs are used to expedite the development even further [2].

Marketing authorization pathways also used to follow a rigid and fixed structure. Only in exceptional circumstances could a drug be approved before completing phase III clinical trials with large number of patients (hundreds to thousands). The available conditional approvals were not used often and mainly for medicines that did not make it through the normal approvals pathway [3]. Some countries implemented methods to allow very early patient access to unapproved medicines, if physicians thought they could be beneficial for individual patients; an example is the German 'Heilversuch' (named patient access) approved for each individual patient by the national medicines agency [4].

\section{New Approaches}

The European Medicines Agency (EMA), Food and Drug Administration (FDA), Medicines and Healthcare Products Regulatory Agency (MHRA) and other medicines agencies have recently developed innovative marketing authorization pathways to facilitate early patient access to innovative and promising new medicines, in order to shorten the long time some patients have to wait until they are able to access new effective medicines (fig. 1). These pathways are either fully implemented, such as the Fast Track, Breakthrough or Accelerated Approval methods by the FDA, and the Early Access to Medicines Scheme (EAMS) by the MHRA, or in a pilot phase, such as the 'Medicines Adaptive Pathways to Patients' (MAPPs) by the EMA [3]. These new approaches are very well defined in each regulator's website and will not be further described in this article. 


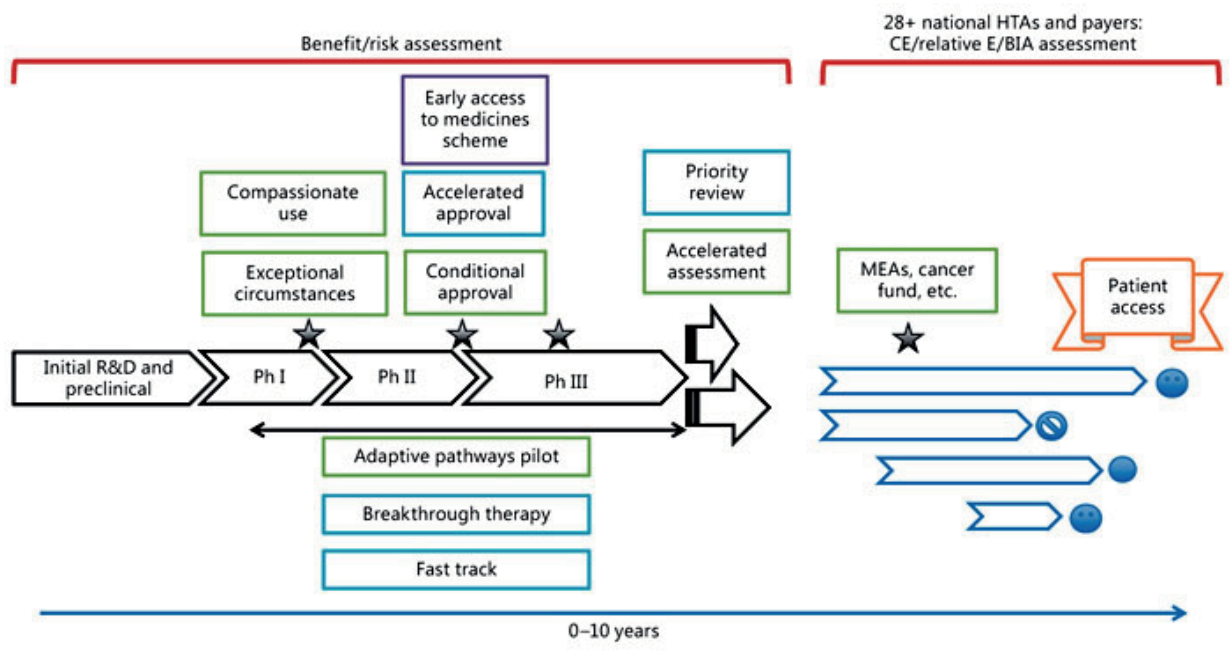

Figure 1. Available regulatory tools for early dialogue and early patient access placed on the current drug development phases from initial R\&D to the market. Green and purple boxes are for available programs in the EU and blue boxes are for available programs in the USA. Modified from [3]. BIA = Budget impact analysis; $\mathrm{CE}=$ cost effectiveness; $\mathrm{MEAs}=$ managed entry agreements; $\mathrm{Ph}=$ clinical trial phase; $R \& D=$ research and development; Relative $\mathrm{E}=$ relative effectiveness.

\section{Common Denominators}

These pathways encourage an early and continuous dialogue between all stakeholders, including manufacturers, applicants, regulators, patients and physicians. For example, the adaptive pathways pilot launched by the EMA in 2014 promotes early discussions between the developer and the agency, as well as early involvement of patients and health technology assessment (HTA) bodies. Real-life evidence that supplements clinical trial data is also a common denominator in many of these pathways. Regulators are starting to accept to review real-life data in innovative marketing authorization application (MAA) methods. The iterative development of medicine is another new common factor; it can be achieved by either approving the medicine in stages with restricted populations at first, or by using conditional approvals and surrogate endpoints that have to be confirmed with more mature data later on [5].

A good example of multi-stakeholder dialogue is the EMA-HTA Scientific Advice procedure. Regulators, manufacturers, HTA bodies, patients, physicians and other experts discuss the questions raised by the manufacturer in one meeting. The patient's and expert's points of view are taken into account and considered by all other parties. At the national level, the British EAMS also promotes early multistakeholder dialogue between the manufacturer, MHRA, NICE and the NHS [6]. 


\section{Differences in Approval Times and Need for New Flexible HTA Methods}

In order to illustrate the difference in effective patient access to medicines in Europe, we will present the case of two cancer drugs: pertuzumab (Perjeta ${ }^{\mathrm{TM}}$ ) and bosutinib (Bosulif ${ }^{\mathrm{rm}}$ ). The delay in the publication of an HTA/reimbursement decision after EC approval, which directly affects patient access, could be up to 18 months in these examples. Furthermore, each national HTA body imposed different restrictions and even had completely different conclusions on the acceptability of the drug for their national patients. The paper by Martinalbo et al. [7] discusses in great detail the differences in pricing and reimbursement decisions and approval times at the national level.

Pertuzumab was approved in March 2013 for patients with metastatic breast cancer and in July 2015 for the neoadjuvant treatment of patients with breast cancer. The orphan drug bosutinib was conditionally approved in March 2013 for patients with Philadelphia chromosome-positive chronic myelogenous leukaemia. As we can see in figure 2, AIFA was the body with longer decision delays in these examples. In the case of pertuzumab, NICE ruled a preliminary decision of not recommending the drug, in contrast to G-BA who recommends its use and suggests that it provides considerable added value in one of its indications. For bosutinib, NICE issued a negative opinion and it is re-examining the evidence within a rapid re-consideration process due to end in October 2016. All other bodies recommended bosutinib even if they agreed that the added value could not be quantified based on the limited information available at the time of MAA.

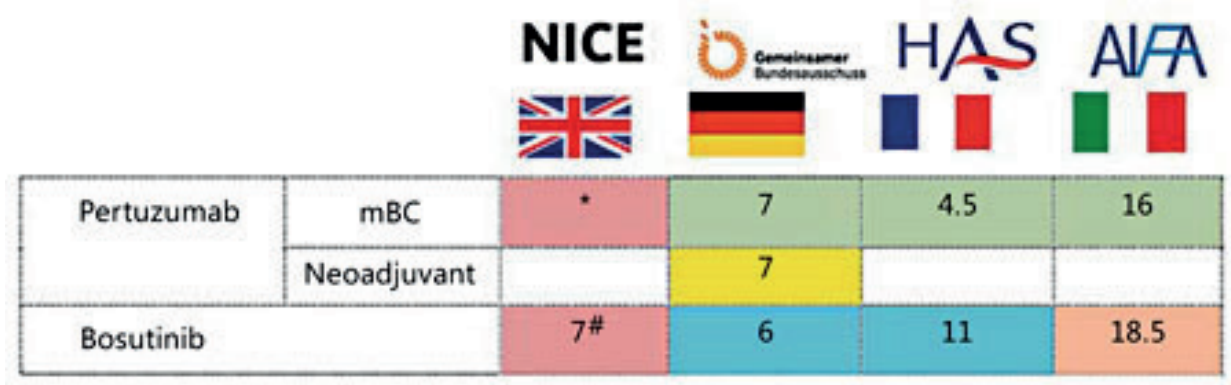

Figure 2. Pricing and reimbursement decisions for pertuzumab (both indications have been separated) and bosutinib in England, Germany, France and Italy. Numbers refer to the delay in decision by national bodies after EC approval (in months). Red = Not recommended; green = recommended with added value; yellow = recommended, no added value; blue = recommended, added value not quantifiable; orange $=$ reimbursed no information on added value; white $=$ no decision yet; $\mathrm{mBC}=$ metastatic breast cancer; $^{*}=$ no final decision has been reached by NICE; the preliminary decision from August 6, 2013 does not recommend pertuzumab; a special committee is studying this and similar cases; \# = bosutinib was in a rapid reconsideration process at the time of article publication with decision publication expected by October 2016. Modified from [5]. 
We have to consider the vast differences in the methods used by these bodies to evaluate medicines. The biggest difference between the bodies evaluated in this paper is the fact that NICE uses cost-effectiveness analysis for its evaluations, whereas G-BA, HAS and AIFA perform a relative effectiveness analysis. This can be one of the reasons for the disparities in final decisions. Whatever the methods are, the truth is that these differences directly affect patient access in Europe, in these cases to the detriment of English patients.

\section{New Proposals for HTAs and Conclusion}

As we have seen in figure 2, the pricing and reimbursement landscape in Europe is too heterogeneous. There are many other examples, and an analysis of many approved drugs would give the same picture. This directly affects patient access. Unfortunately, the efforts from the EMA and other regulatory agencies to facilitate early patient access to innovative medicines are 'diluted' in the pricing and reimbursement (P\&R) and HTA process by long delays.

Even though they constitute big steps forward, the 'early dialogue' and the 'multistakeholder dialogue' promoted by the MAPPs pilot and the EMA-HTA scientific advice procedure are voluntary procedures and are not implemented in every medicine's development. The ideal future situation would entail the inclusion of systematic early and multi-stakeholder dialogue in all medicine developments that have reached certain milestones and prove to be promising.

It is clear that patients' safety should be at the forefront of all stakeholders' intentions. As some critics of flexible approval methods are expressing their concerns on these methods and arguing that they endanger patients' safety, it is important to remind them that regulatory agencies know their mandates and discuss in great detail all decisions, always having the patients' best interest in mind. We have to be able to find the right balance between acceptable early evidence for determining a positive risk/benefit balance and the risks associated with limited information. If the new flexible approval pathways allow restricted and controlled patient access, this risk can be well controlled and immediate actions can be taken when safety signals appear.

To avoid the delay in patient access and the European 'post-code' lottery, we need a more coordinated HTA process at the European level. As the ENVI report [7] proposes, relative effectiveness could be evaluated centrally with a common conclusion that is then applied nationally or locally by each country or region according to its applicable priorities and regulations. Another important aspect is to link the HTA and the MAA processes. They evaluate very similar information on individual products; it is therefore a waste of resources that all HTA/reimbursement bodies re-analyze the same information the medicine 
agencies have already studied in detail. In this direction, the EMA is considering the possibility to include more HTA-relevant information in their CHMP assessment report in order to facilitate the work of their HTA colleagues and the reimbursement evaluation process [8]. But most importantly, HTA and reimbursement bodies have to leave behind the rigid evaluation methods and adopt the flexible methods that are being implemented by medicine agencies. This is the only way to offer patients an effective early access to medicines. Nevertheless, we have to take into account that HTA bodies are 'younger' bodies than medicine agencies; it is therefore possible that they need more time to reach the 'maturity' and experience that medicine agencies needed in order to start implementing flexible approaches that force them out of the comfort zone of nearly total assurance in their risk/benefit decisions.

As essential next steps, we can consider the need to provide more insights into improving HTA techniques on the level of research. Furthermore, we need to extend the reach of HTA to all other aspects of health, beyond medicines and medical devices. On the European political level, we need a unified approach for the determination of relative effectiveness for medicines and medical devices, and most importantly, on the policy level, we need new flexible approaches in order to catch up with the ones from medicine agencies to facilitate early patient access. The important goal of early patient access to promising medicines in Europe can only be achieved if the national health technology assessment bodies, such as NICE (ENG), HAS (FR), G-BA (DE) or AIFA (IT), provide harmonized, transparent, flexible, conditional and adaptive methods that adopt the level of evidence accepted by the medicine agencies. The efforts from medicines agencies are essential but will be in vain if HTAs do not follow with similar initiatives, and the European 'post-code' lottery will continue. 


\section{Chapter 5}

\section{References}

1. Adams C, Bran V: Spending on new drug development. Health Economics 2010;19:130-141. External Resources

2. Berntgen M, Gourvil A, Pavlovic M, Goettsch W, Eichler H-G, Kristensen F: Improving the contribution of regulatory assessment reports to health technology assessments - a collaboration between the European Medicines Agency and the European network for Health Technology Assessment. Value Health 2014;17:634-641. External Resources

3. European Parliament: Towards a Harmonised EU Assessment of the Added Therapeutic Value of Medicines. Policy Department A: Economic and Scientific Policy. Brussels, European Union, 2015.

4. Hart D: Heilversuch und klinische Prüfung. Kongruenz und Differenz. Medizinrecht 2015;33:766775. External Resources

5. Leyens L, Richer É, Melien $\emptyset$, Ballensiefen W, Brand A: Available tools to facilitate early patient access to medicines in the EU and the USA: analysis of conditional approvals and the implications for personalized medicine. Public Health Genomics 2015;18:249-259. External Resources

6. Lockwood P, Ewy W, Hermann D, Holford N: Application of clinical trial simulation to compare proof-of-concept study designs for drugs with a slow onset of effect; an example in Alzheimer's disease. Pharm Res 2006;23:2050-2059. External Resources

7. Martinalbo J, Bowen D, Camarero J, Chapelin M, Démolis P, Foggi P, et al: Early market access of cancer drugs in the EU. Ann Oncol 2016;27:96-105. External Resources

8. NICE: Note to describe procedures at NICE to support the early access to medicines scheme. https://www.nice.org.uk/Media/Default/About/Who-we-are/Policies-and-procedures/eamsprocess-jan-16.pdf (accessed April 10, 2016). 


\section{Chapter 6}

\section{Use of Big Data for drug development and for Public and Personal Health and Care}

Published as: Leyens L., Reumann M., Malats N., Brand A. Use of Big Data for drug development and for Public and Personal Health and Care. Genetic Epidemiology 2017 Jan; 41(1):51-60 (doi: 10.1002/gepi.22012). Epub 2016 Nov 21 


\section{Chapter 6}

\section{Abstract}

The use of data analytics across the entire healthcare value chain, from drug discovery and development through epidemiology to informed clinical decision for patients or policy making for public health, has seen an explosion in the recent years. The increase in quantity and variety of data available together with the improvement of storing capabilities and analytical tools offer numerous possibilities to all stakeholders (manufacturers, regulators, payers, healthcare providers, decision makers, researchers) but most importantly, it has the potential to improve general health outcomes if we learn how to exploit it in the right way. This article looks at the different sources of data and the importance of unstructured data. It goes on to summarize current and potential future uses in drug discovery, development and monitoring as well as in public and personal healthcare; including examples of good practice and recent developments. Finally, we discuss the main practical and ethical challenges to unravel the full potential of big data in healthcare and conclude that all stakeholders need to work together towards the common goal of making sense of the available data for the common good. 


\section{Introduction}

"Without the right analytical methods, more data just gives a more precise estimate of the wrong thing" [Ellenberg 2015].

The use of data in public health applications and in drug development and monitoring has been part of standard practice for a very long time. Epidemiologists have studied the patterns, causes and effects of health and disease in populations for decades combining different type of data to do so [Chiolero 2013]; biologists have evaluated data from complex biological systems to develop predictive models of disease for decades [Boyle 2013]; biostatisticians have analysed clinical trial data to confirm or reject the studied hypothesis since the 1950s (the first randomized controlled trial of streptomycin was developed in 1946) [Bhatt 2010]; etc. So the following questions arise: "What is different now? Why is a there a sudden interest and hype in the use of 'big data' for healthcare?"

The new potential does not lay in the use of data per se but on the increased amount of information we are gathering in all fields and more importantly on the increased accessibility and 'exploitability'. New tools are being created to store, structure, analyse and exploit these data; and the advances in technological capabilities enable more effective and smarter analysis of larger quantities of data simultaneously. All these aspects combined allow for the analysis and combination of new types of information (e.g. social media) that previously could not have been imagined to play a role in public health, drug development and monitoring [Broniatowski, et al. 2014].

This increased wealth of data is coming from many different sources and developments in areas that are not linked to each other, and not necessarily linked to health (see table 1 for a snapshot). Some examples are the wider implementation of electronic health records (EHRs) in hospitals and healthcare providers; the faster and cheaper DNA sequencing methods that are delivering large amounts of genetic information daily; the vast information shared by patients and citizens through social media and the web; readings from remote sensors and devices measuring vital signs; data from insurance claims and billing records (depending on each regional/national health system); data obtained from the increasing number of diagnostic tools and biomarkers available for all therapeutic areas; and in general unstructured data from e-mails, notes, text messages, paper documents, etc.

However, the simple fact that there is more data is not useful to public health unless we are able to turn it into 'actionable big data' for improved health outcomes and more effective and efficient health systems. It will only inform public health decision making if we can make meaningful extrapolations and inferences, ensuring we are quantifying the likelihood of errors to avoid offering false positive 
evidence [Jordan and Mitchell 2015]. In addition to the 3Vs of big data -Volume (high quantity of data), Variety (very different categories of data) and Velocity (fast data generation) - its Veracity (quality of the data) and its Value (how useful is the data) are paramount to unleash the potential for big data use in healthcare, translational science and public health [Belle, et al. 2015].

Regulators and public health decision makers have also started using novel data sources and advanced analytical methods to help them in the decision making process or in the monitoring of approved drugs and implemented interventions [Szezák, et al. 2014]. In the following sections we will analyse the current and potential applications for big data in health, focusing on drug development, public health and personal health.

Table 6-1. Potential type of data in EU health care (modified from [Szezák, et al. 2014] for the EU setting). The main source may greatly vary depending on national health care systems and national or regional arrangements. HCP: Healthcare Providers. EHRs: Electronic Health Records

\begin{tabular}{|c|c|c|c|c|}
\hline Type & & Description & Main source & $\begin{array}{l}\text { Structured/ } \\
\text { unstructured }\end{array}$ \\
\hline \multirow[t]{3}{*}{ Clinical } & $\begin{array}{l}\text { Electronic Health } \\
\text { Records (EHRs) }\end{array}$ & $\begin{array}{l}\text { Patient level clinical } \\
\text { data }\end{array}$ & $\begin{array}{l}\text { Hospitals and } \\
\text { healthcare } \\
\text { providers }\end{array}$ & $\begin{array}{l}\text { Structured, } \\
\text { unstructured }\end{array}$ \\
\hline & Registries & $\begin{array}{l}\text { Basic medical } \\
\text { information specific to } \\
\text { one disease/therapeutic } \\
\text { area }\end{array}$ & $\begin{array}{l}\text { Patient/health } \\
\text { associations }\end{array}$ & Structured \\
\hline & $\begin{array}{l}\text { Diagnostics and } \\
\text { biomarkers }\end{array}$ & $\begin{array}{l}\text { Results from diagnostic } \\
\text { tests and biological } \\
\text { indicators of disease } \\
\text { status or treatment }\end{array}$ & EHRs & Structured \\
\hline \multirow[t]{4}{*}{$\begin{array}{l}\text { Health care } \\
\text { utilisation }\end{array}$} & Insurance claims & $\begin{array}{l}\text { Medical information and } \\
\text { health utilisation } \\
\text { information from claims } \\
\text { (depending on national } \\
\text { health care system) }\end{array}$ & $\begin{array}{l}\text { Public or private } \\
\text { insurance } \\
\text { providers }\end{array}$ & $\begin{array}{l}\text { Structured, } \\
\text { unstructured }\end{array}$ \\
\hline & $\begin{array}{l}\text { Admission, } \\
\text { discharges }\end{array}$ & $\begin{array}{l}\text { Summary of } \\
\text { administrative } \\
\text { information }\end{array}$ & $\begin{array}{l}\text { Hospitals and } \\
\text { healthcare } \\
\text { providers (HCPs) }\end{array}$ & Structured \\
\hline & $\begin{array}{l}\text { Drug orders and } \\
\text { sales }\end{array}$ & $\begin{array}{l}\text { Records of drugs sales } \\
\text { and revenue }\end{array}$ & $\begin{array}{l}\text { Distributors, } \\
\text { pharma companies, } \\
\text { HCPs, pharmacies }\end{array}$ & Structured \\
\hline & $\begin{array}{l}\text { Clinical and } \\
\text { pharmacy dispensing }\end{array}$ & $\begin{array}{l}\text { Dispensing data from } \\
\text { medications } \\
\text { administered in } \\
\text { hospitals and } \\
\text { pharmacies }\end{array}$ & HCPs, pharmacies & $\begin{array}{l}\text { Structured, semi- } \\
\text { structured }\end{array}$ \\
\hline Biological & '-omic' information & $\begin{array}{l}\text { Not related to clinical } \\
\text { diagnosis (e.g. from } \\
\text { biomedical research } \\
\text { projects) }\end{array}$ & $\begin{array}{l}\text { Research } \\
\text { institutions, } \\
\text { international } \\
\text { molecular } \\
\text { databases }\end{array}$ & $\begin{array}{l}\text { Structured, } \\
\text { unstructured }\end{array}$ \\
\hline
\end{tabular}




\begin{tabular}{|c|c|c|c|c|}
\hline Type & & Description & Main source & $\begin{array}{l}\text { Structured/ } \\
\text { unstructured }\end{array}$ \\
\hline & $\begin{array}{l}\text { Other biological } \\
\text { information }\end{array}$ & $\begin{array}{l}\text { Data from biomedical } \\
\text { research }\end{array}$ & $\begin{array}{l}\text { Research } \\
\text { institutions, } \\
\text { international } \\
\text { molecular } \\
\text { databases }\end{array}$ & $\begin{array}{l}\text { Structured, } \\
\text { unstructured }\end{array}$ \\
\hline \multirow[t]{2}{*}{$\begin{array}{l}\text { Drug } \\
\text { development }\end{array}$} & Clinical research & $\begin{array}{l}\text { Clinical trial design } \\
\text { parameters and results }\end{array}$ & $\begin{array}{l}\text { Pharmaceutical } \\
\text { companies, } \\
\text { regulators, } \\
\text { international } \\
\text { clinical trial } \\
\text { repositories, } \\
\text { biomedical journals }\end{array}$ & $\begin{array}{l}\text { Structured, } \\
\text { unstructured }\end{array}$ \\
\hline & $\begin{array}{l}\text { Safety and } \\
\text { Pharmacovigilance }\end{array}$ & $\begin{array}{l}\text { Adverse drug reactions } \\
\text { (serious / non serious) } \\
\text { before and after } \\
\text { marketing authorisation }\end{array}$ & $\begin{array}{l}\text { Pharmaceutical } \\
\text { companies, } \\
\text { regulators, } \\
\text { international } \\
\text { repositories }\end{array}$ & $\begin{array}{l}\text { Structured, } \\
\text { unstructured }\end{array}$ \\
\hline \multirow[t]{2}{*}{$\begin{array}{l}\text { Patient- } \\
\text { generated } \\
\text { data }\end{array}$} & Social media & $\begin{array}{l}\text { All type of information } \\
\text { on health from patients } \\
\text { and physicians in online } \\
\text { communities, facebook, } \\
\text { twitter, etc. }\end{array}$ & $\begin{array}{l}\text { Websites, blogs and } \\
\text { smartphone apps }\end{array}$ & Unstructured \\
\hline & Monitoring devices & $\begin{array}{l}\text { eHealth/mHealth } \\
\text { monitoring devices and } \\
\text { sensors that measure } \\
\text { vital signs and other } \\
\text { aspects }\end{array}$ & Monitoring devices & \\
\hline \multirow[t]{2}{*}{ Other } & $\begin{array}{l}\text { Scientific } \\
\text { publications }\end{array}$ & $\begin{array}{l}\text { Scientific discoveries } \\
\text { published in scientific } \\
\text { literature }\end{array}$ & $\begin{array}{l}\text { Biomedical } \\
\text { journals, books, etc. }\end{array}$ & Unstructured \\
\hline & Epidemiological data & $\begin{array}{l}\text { Various data on } \\
\text { diseases, health and } \\
\text { environment }\end{array}$ & $\begin{array}{l}\text { Governments, } \\
\text { researchers, } \\
\text { various } \\
\text { organisations }\end{array}$ & $\begin{array}{l}\text { Structured, } \\
\text { unstructured }\end{array}$ \\
\hline
\end{tabular}

\section{Structured vs, unstructured data uses}

The different types of data (see table 1) can be classified under structured, semistructured and unstructured data [Martin-Sanchez and Verspoor 2014]. Specific examples of structured and unstructured data are listed in Table 2.

Structured data refers to information that is highly organised and can be searchable by simple and straightforward algorithms and search operations. This type of data has been used for a very long time in drug development and public health. The biggest difference nowadays is the amount of data we produce, we can store and analyse. As examples of the new applications, we have seen the advances in genomic sequencing and other '-omic' fields produce new sources of data that can help biomedical researchers unravel new hypotheses on the molecular causes of disease [Wang et al. 2013] and an increased number of data from registries and 
clinical records are analysed to evaluate health interventions and their impact [Jalali et al. 2012], between others.

Table 6-2. Examples of structured and unstructured data. EPAR: European public assessment reports released by the European Medicines Agency; PSURs: Periodic Safety Update Reports

\begin{tabular}{lll}
\hline & Structured & Unstructured \\
\hline $\begin{array}{l}\text { Molecular and biological } \\
\text { information }\end{array}$ & $\begin{array}{l}\text { Databases with data on DNA, } \\
\text { RNA, gene expression, proteins, } \\
\text { structures, systems, chemical } \\
\text { biology, ontologies, etc. }\end{array}$ & $\begin{array}{l}\text { Scientific literature, annotations } \\
\text { and linkages, written notes, } \\
\text { conference proceedings, etc. }\end{array}$ \\
Clinical / Healthcare information & $\begin{array}{l}\text { EHRs, databases from health } \\
\text { care provider administrative } \\
\text { information, filled forms for } \\
\text { insurance claims, information on } \\
\text { diagnostics, etc. }\end{array}$ & $\begin{array}{l}\text { Free text in EHRs, insurance } \\
\text { claims and medical notes }\end{array}$ \\
& $\begin{array}{l}\text { Clinical Trial repositories, } \\
\text { databases with clinical trial data }\end{array}$ & EPARs, biomedical journals \\
Drug development & $\begin{array}{l}\text { International adverse event } \\
\text { repositories, clinical trial data, } \\
\text { Safety and Pharmacovigilance }\end{array}$ & $\begin{array}{l}\text { PSURs, information from social } \\
\text { media }\end{array}$ \\
& $\begin{array}{l}\text { Weather and contamination } \\
\text { databases, disease outbreak } \\
\text { Eatterns, socioeconomic factors, } \\
\text { lifestyle, etc. }\end{array}$ & $\begin{array}{l}\text { Historical information contained } \\
\text { in books and periodicals }\end{array}$ \\
& & \\
\hline
\end{tabular}

However, one of the new areas that are now being explored is the use of large amounts of unstructured data for drug development, drug monitoring and public health. This is possible through the increased use of social media, the rise of information shared by patients and citizens through various platforms and mainly due to the expansion of technology capabilities that allow exploiting this data. When we refer to unstructured data, we refer to information that does not fit a pre-defined model or is not organised in a pre-defined manner (e.g. free text entries). The advantage of unstructured data is that information can be uncovered that is extremely relevant for the diagnosis and treatment planning. For example, taking a patient history might include filling out a questionnaire with a tick box whether the patient is a smoker or a non - smoker. While the patient might tick the "non - smoker" box, the medical doctors report on the patient history might reveal a side remark the patient made that he had just stopped smoking a couple of month ago after 25 years of smoking. Thus, unstructured information can be used to verify the veracity of structured data. Furthermore, it can contain information about risk factors that allow the prediction of re-admission of patients in e. $\mathrm{g}$. congestive heart failure [Feldman, et al. 2012]. Social media threads and Internet searches are another form of unstructured data that is generated at high speeds, high volumes and its veracity is often unknown. Its use and potential in public health has been widely recognized [Milinovich 2015, O'Donovan 2015]. What makes the use of unstructured data with today's technological capabilities so 
interesting is that its current and future purposes within and beyond the health sector can be unknown at the time of data storage. In pharmacovigilance activities, for example, data from social media could cover the deficient patient self-reporting of side effects through official channels [Broniatowski, et al. 2014]; which was not considered a potential use for social media data when it started being produced.

\section{Uses for big-data in health: drug development}

The potential use of big data in drug development lies in the discovery and development phase and in the post-marketing monitoring of drugs and devices, especially in safety monitoring and pharmacovigilance (see figure 1). In this section, we explore these applications and also see why the use for regulatory purposes, outside the scope of pharmacovigilance and post-marketing activities, is currently limited or non-existent.

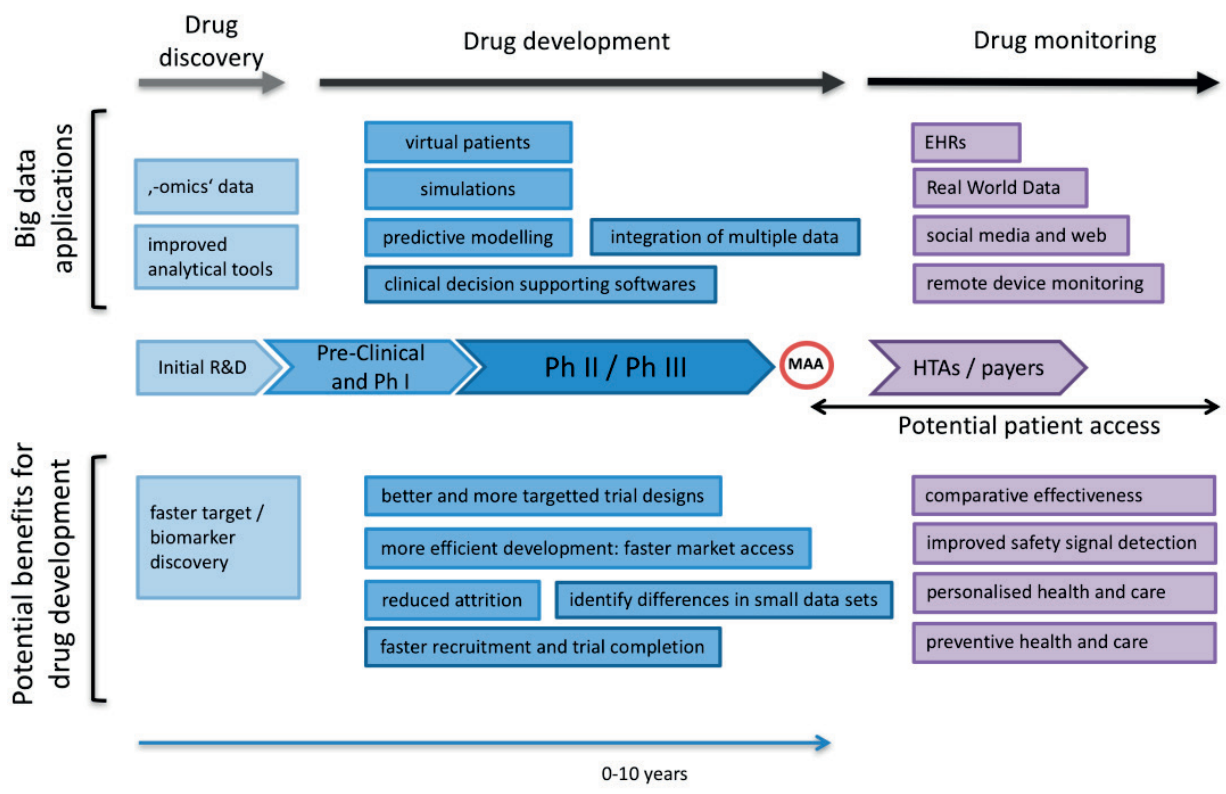

Figure 1. This figure illustrates the potential benefits of big data for drug development. Big data applications (top boxes) and potential benefits (bottom boxes) are divided according to the lifecycle of drug development (middle axis), from conception (drug discovery) to patient access (drug monitoring). EHRs: electronic health records; HTAs: health technology assessment bodies; MAA: marketing authorisation application; Ph I / II / III: phase I / II / III clinical trials; R\&D: research and development.

\section{Drug discovery}

Researchers nowadays have access to larger sets of data thanks to the rapidly improving analytical methods that are able to produce genomic, transcriptomic, proteomic, metabolomics and other type of biological data faster and cheaper than 
ever before. Since Sanger and Coulson published their first sequencing procedure 30 years ago [Sanger and Coulson 1975], we have developed high throughput sequencing machines that are able to map human genome sequences in less than a week and more than 22,000 times cheaper than in 2001 (see figure 2) [Wetterstrand 2015]. The European Bioinformatics Institute (EMBL-EBI) stores more than 20 petabytes ( 1 petabyte $=10^{15}$ bytes) of biological data and is one of the World's largest biology data repository, which can be accessed by researchers to conduct their analysis [Nature 2013].

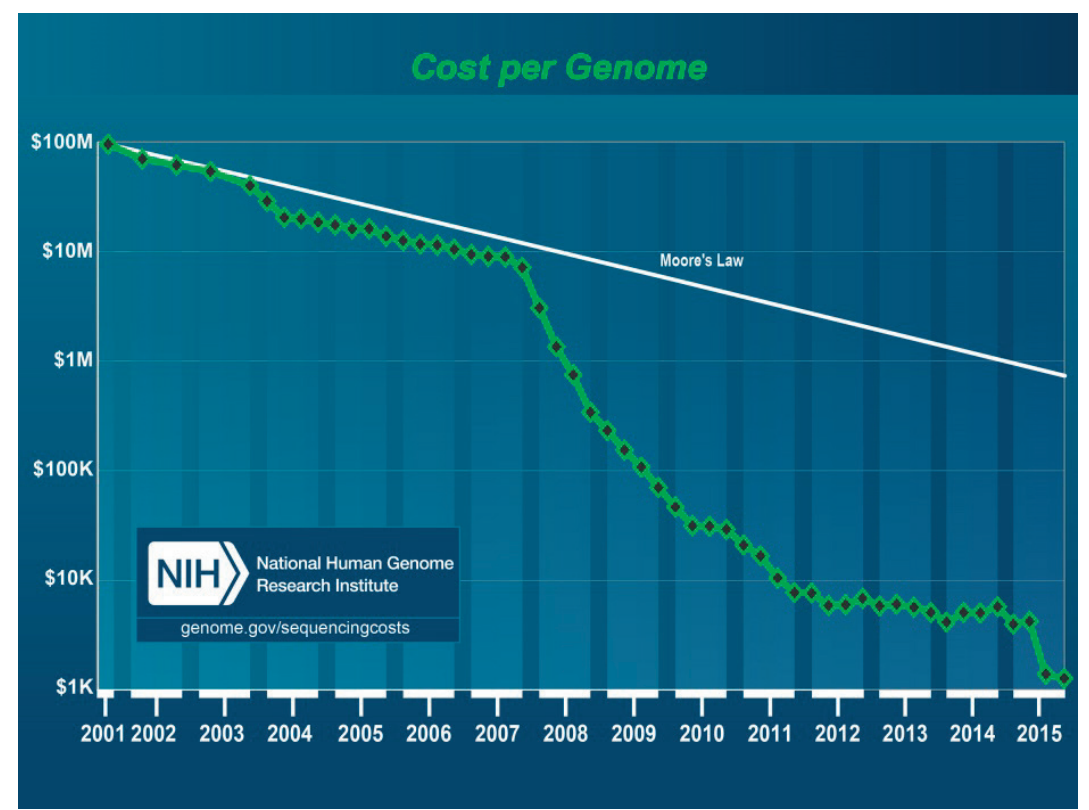

Figure 2. Decline in cost per genome (of human size) since 2001. From [Wetterstrand 2015].

Integration of this wealth of '-omic' data together with the right analytical tools and the right research question or hypothesis can provide insight into associations and linkages that allow for faster drug target discovery and biomarkers for drug discovery [Szezák, et al. 2014]. The areas of pharmacogenomics and personalised medicine are potential candidates that will benefit from this type of big data based research. Some public-private partnerships (PPPs) in the EU and the USA are using the big data approach for this purpose, e.g. the NIH-led PPP AMP has just launched a Big Data portal for Alzheimer's drug discovery [Sage Bionetworks 2015]. We have not seeing dramatic applications of this concept in drug development yet [Horgan, et al. 2014], with the usual time to develop pharmaceuticals being close to 10 years we should analyse after 2020 whether the expectative for big data in this area has materialised. 


\section{Drug development: $R \& D$}

The potential benefits of 'rational' big data for the stakeholders involved in drug development (all stages after drug discovery) and ultimately for patients are very large. A more efficient and targeted R\&D process can translate into new medicines reaching the market faster and being (in theory) cheaper. Big data can facilitate $R \& D$ efforts in different fronts, from predictive modelling that lowers attrition to the improvement of clinical trial designs (e.g. endpoints, inclusion/exclusion criteria, etc.) that reduce trial failure and trial costs (reduced number of amendments and shorter completion times) [Raghupathi and Raghupathi 2014].

Virtual patients, created from EHRs, pharmacokinetic and pharmacodynamic data and historical data can help power clinical trials for regulatory submission. In a recent example, the inclusion/exclusion criteria for a clinical trial assessing a new type 2 diabetes treatment were defined using information from a simulated trial that was run based on virtual patients [Garrett, et al. 2015]. Another development we have seen in the past years is the ability of clinical decision supporting systems (CDSS), based on machine learning, to identify candidate patients for clinical trials (e.g. IBM Watson Health's Clinical Trial Matching solution) [IBM 2014] [Lehrach H 2015]. These programs have the ability to compare patients' medical and clinical information with the inclusion/exclusion criteria of recruiting clinical trials, combining information from numerous sources (e.g. EHRs, biomarkers and international clinical trial repositories) to identify suitable candidates for participation in these trials. This can speed up the recruitment and completion of clinical trials, especially those that cover diseases with very small population pools. Various studies have evaluated the efficacy and efficiency of applying CDSS in clinical trial recruitment [Köpcke et al. 2014]. Time-sensitive clinical trials in Intensive Care Units (ICU) can really profit from this technology since the usual impediment of late notification and delayed recognition hamper recruitment in these trials. Herasevich and colleagues report about an automated HER screening process that produced alerts when eligible patients entered the ICU resulting in improved enrolment efficiency [Herasevich et al. 2011].

Analysis of large quantities of healthcare data (e.g. clinical trial data and EHRs) also has the potential to identify new pharmacogenomics interactions and additional indications for already existing drugs, for new drug candidates and for drugs that have failed in other indications and never reached the market. Furthermore, big data and special statistical methods can assist the development of personalised medicines, where the worldwide patient pools are very limited, by integrating data from numerous trials and facilitating the identification of differences between drugs [The Economist 2015]. 


\section{Drug monitoring}

The area of drug safety monitoring and comparative effectiveness based on 'Real World Data' is where the use of unstructured data from normal clinical practice becomes prominent and where the use of social media is being explored. Structured safety data is collected in international repositories such as VigiBase (WHO), EudraVigilance (EMA) and AERS (FDA) in the form of adverse event reports filled by drug product manufacturers (compulsory), healthcare professionals (voluntary) or patients (voluntary and still very rare). The FDA has recently opened the adverse event report database to facilitate research on safety signals and correlations; reports since 2004 are available in this web-based resource [FDA 2014].

Safety monitoring in clinical practice is very important for all drugs and devices, but it is paramount for personalised medicines and orphan drugs (which receive marketing authorisation based on data from very small patient populations) and for the new regulatory pathways that facilitate earlier patient access to medicines at the expense of higher uncertainties in the risk of these medicines [Leyens, et al. 2015]. Furthermore, rare adverse events may not be identified in clinical trials and only picked up through pharmacovigilance signals or real world data.

To cover the deficiencies in patient self-reporting of adverse events, programs such as the FDA Medwatch aim to increase the structured reporting of safety information. However, new ways of identification of safety signals are needed and social media in combination with other web-resources and remote monitoring devices (or health apps) have opened a new possible pathway. The IMI project WEB-RADR (http://web-radr.eu/) launched in 2014 is evaluating how to identify potential safety signals from medicines through user comments in social media in partnership with FDA and EMA. In June 2015, another collaboration that looks into the use of patient-reported outcomes for drug safety monitoring was announced between FDA and PatientsLikeMe [PatientsLikeMe 2015]. Aspects such as duplication of information, veracity and value will have to be carefully evaluated in such projects.

Real world data is also being increasingly used for comparative effectiveness evaluations and post-marketing data analysis. HTA and reimbursement agencies are starting to accept outcomes data collected and processed from clinical practice in new ways, sometimes non-standardised. Traditional post-market studies could be complemented by information from EHRs, real time remote monitoring devices and data from social media [Szezák, et al. 2014] [Leyens and Brand 2016].

Further regulatory uses, such as submission of big data analytics as pivotal information for marketing authorisation applications, are not possible nowadays and are unlikely in the near future. At present, the regulatory applications are limited to the ones described above: to make drug development more efficient and targeted, to inform the design of pivotal studies, to monitor safety or relative 
effectiveness. Due to the high uncertainty in big data analysis, it can only represent supportive but not pivotal evidence.

\section{Uses for big-data in health: public and personal health}

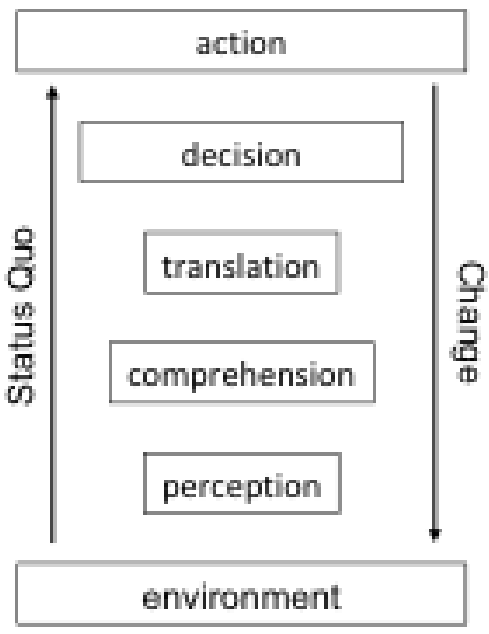

Figure 3. Situational awareness require a person to perceive the environment, process the information to comprehend and translate the data into context and knowledge so that decisions are made and actions can be taken. This process will change the status quo of the environment and situation through situational awareness and acting accordingly.

Public health stakeholders participate in a variety of tasks with different levels of responsibility in the health system to ensure and improve the health of the community [Ola and Sedig 2014]. The stakeholders apply analytical reasoning to the situation to make sense out of the data evidence. In general terms, public health stakeholders require great situational awareness to measure and perceive the environment at a specific given time, understand the data and translate it into information and knowledge to then form a decision and act upon the evidence base and need for action (see Figure 3). The perfect storm of big data in public health demands for technology to make the data transparent and put it into context so that public health stakeholders can apply the cognitive task of understanding and acting upon it.

The aggregation of real world data helps policy makers to guide programs and legislation. Real world data in public health does not include just the classic health related information about an individual patient. It also comprises information about infrastructure, population and other epidemiological factors including even weather information or socioeconomic factors. Visual analytics [Ola and Sedig 2014] and predictive modelling [Davis et al. 2014] is an active area of research that could become an important building block to shape health policies and for public 
health professionals to engage in an agile manner and to predict and react to disease outbreaks [Davis et al. 2014]. The role of big data in the early detection of emerging infectious diseases is becoming more and more apparent in real world scenarios where the role of internet searches in outbreak detection [Milinovich 2015] and mHealth applications, including tracking of text messages from mobile phones [O'Donovan 2015], become essential tools for public health organizations. In fact, telecommunication companies have started engaging in providing data for better public health monitoring and education. All information from laboratory tests to social media and environmental factors should be captured in one system that allows timely action and planning of public health measures. Davis et al. demonstrated how the use of information on disease incidence, population models, weather and geographic information could be used in spatio-temporal epidemiologic computer simulations to predict Malaria incidence and outbreaks as well as how to test intervention strategies and their respective impact on a population level [Davis et al. 2014]. Not only can these systems be used for monitoring and surveillance, making real world data transparent and putting it into context, but they can also inform decision making processes (see Figure 4).

\section{Real world data use to shape health policy}

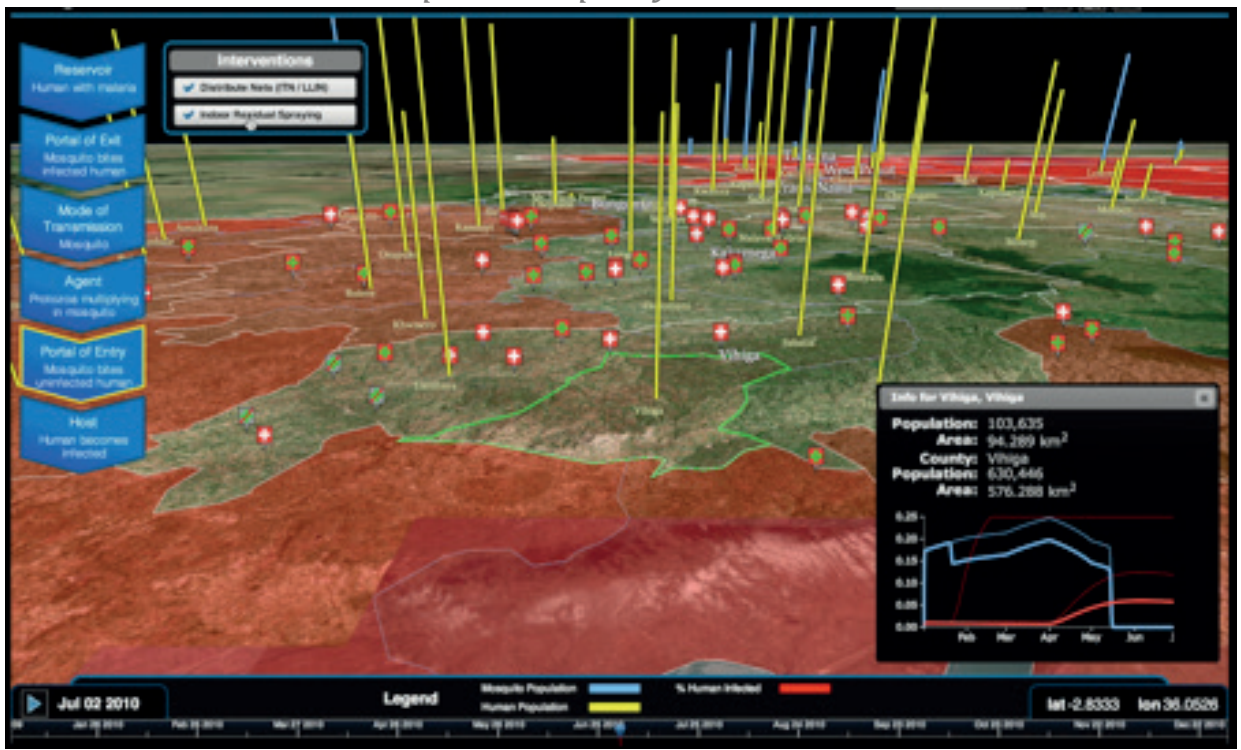

Figure 4. This figure shows the interface of a demonstration of IBM's Cognitive Health Care and Health systems Hub [https://www.youtube.com/watch?v=ABmIeWcmG3E]. In this scenario, malaria and interventions to reduce the disease burden are modelled using the Spatio-Temporal Epidemiological Modeller library [Davis et al. 2014]. The human population and estimated mosquito count for counties in Kenya in 2010 are displayed by yellow and blue bars, respectively. On the left, the chain of causation for malaria is displayed and intervention strategies can be modelled. This illustration of malaria incidence and response has been created for demonstration purposes only and does not reflect actual data collection on malaria and intervention strategies. 


\section{Resilient health systems}

Thus, to build a sustainable, resilient health system, one must consider all sources of information captured through diverse means (e.g. mobile/social, medical professionals, wearables), integrate and analyze that data by harnessing cognitive computing to deliver the right information, insight and knowledge for the right patient to the right care giver and medical professional. Such a resilient health systems solution can then not only integrate medical and environmental information but also data on infrastructure, resources and supplies as well as work force including skill distribution to direct the right care path for each individual patient. This concept does not only apply to developing nations, like African countries, where a clean water supply would dramatically improve the health of the population and where sustainable health systems need to be established; but also to Western nations, like the USA and European countries, where established health systems are bound to fail due to increasing costs that make health care not sustainable.

In this context, building a resilient health system that is empowered by cognitive computing and founded not only on health data but furthermore integrating environmental, geographic and population data will create a paradigm shift in combatting diseases. The holistic view will cover the whole pipeline from data capture from a variety of sources, processing, advanced big data analytics and predictive modeling as well as knowledge visualization and stakeholder interaction. It therefore must include the following three components:

1. End - to - end computer system for diverse data capture and integration,

2. Advanced, contextual analysis and predictive modelling build on cognitive computing with the ability to learn to become an expert system, and

3. Intuitive knowledge visualization and stakeholder interaction to intuitively deliver data, information and knowledge in the health systems context.

The impact of such a system can be illustrated by looking at the antimicrobial resistance (AMR) challenge. It exemplifies the need to translate research and routine tests in the laboratory (bench) that leverages next generation genome sequencing for pathogen identification to clinical practice (bedside) and to further integrate that information in infectious disease monitoring and surveillance system (society) together with the (antibiotic) treatment chosen so that public health professionals can use this information to improve treatment options and by this health outcomes and the well-being of society. Most action plans and public health initiatives to combat AMR include monitoring and tracking of pathogens, their genomes and their resistances as well as education of antibiotics use. Slowly, research actions are also supported to discover new antimicrobials and to improve the clinical and public health management of AMR. To achieve this goal, harnessing 
big data research and technologies will create a paradigm shift in combatting AMR by addressing the root cause of AMR for discovery and translation into actionable information and knowledge. A key concept here is to turn big data into 'actionable big data' meaning that the collection and capture of data is of no consequence if the stakeholders cannot take action on it to improve the situation (see Figure 3).

\section{Comparative effectiveness research and best practices}

The integration of big data into a single system that allows a unified data view also facilitates clinical research to advance our understanding of disease and furthermore the best treatment and preventive options for the individual patient. In particular, comparative effectiveness research (CER) enables the comparison of benefits and risks of treating a particular set of patients with alternative methods [Zhang et al. 2013]. In addition of the aforementioned use of CER in the drug development process, it is used to monitor, prevent, diagnose and treat patients and to improve the delivery of care. The advantage of CER is that the success of an intervention is evaluated in the real-world setting versus controlled randomized clinical trials that measure efficacy under well-controlled, ideal clinical conditions. CER is critical for providing evidence and support for informed medical care and health policy decision making on a dynamic, daily basis that adapts to the fast pace of clinical care and accelerated translation of research discoveries into clinical practice.

Visual analytics and interactive mining of data plays an important role to comprehend the information and to create hypothesis that can be tested statistically and through other scientific or differential diagnostic means. The goal is to make patient data, information and knowledge about a disease and its treatment transparent. Clinical event patterns and pathways can be extracted from electronic health records or clinical event logs [Gotz et al. 2014, Huang et al. 2014]. They can then be compared with clinical pattern of other patients that underwent the same or similar treatment with a particular focus on treatment outcomes. Patient similarity can even be extracted from heterogeneous patient records [Sun et al. 2012] so that integration of a variety and diverse data sources can be achieved and geared towards clinical impact. As an example, an EHR data mining algorithm was able to present questionable prescribing patterns in a cohort of hyperlipidemia patients who where occasionally given medications with side effects that have been associated in the medical literature with raising LDL levels [Perer et al. 2015]. As such, these tools can be used to identify best practices and improve health care delivery. It becomes a true win - win situation. Patients will receive more appropriate preventive care and treatment for their conditions and consequently stay healthier. This in turn has an impact on the economy of the health system that becomes less burdened with reduced costs. Medical doctors benefit directly from such a system as they can make real-time treatment decisions that are based on the best evidence available. Furthermore, policy makers can use 
the aggregated information to speed up decision on medical guidelines, knowledge dissemination and funding models.

\section{Challenges}

Many papers and reports already discuss the challenges for the application in healthcare [Science Europe 2014, European Health Forum Gastein 2013, PerMed 2020 CSA 2015], we summarise below the ones we consider crucial.

Some of these challenges are structural and inherent to a system where so many different types of data from so many varied sources come into play. All the data we store and analyse is heterogeneous, the same type of data stored in different databases may differ greatly in aspects such as accuracy, format and detail. This data is also greatly fragmented, it is stored in numerous unconnected data sources controlled by multiple stakeholders; this is commonly referred to as information silos. It is not only the unconnected data sources that pose a challenge; the segmentation of the current biomedical research model into basic, pre-clinical, clinical research silos and the healthcare model with unconnected structures for primary, secondary, tertiary and social care also contribute to the information silos we face. Furthermore, all data is not available to all stakeholders; this may be due to intellectual protection for commercial purposes, personal privacy, cultural and language barriers or simple lack of transparency and awareness that a certain database exists. In addition, data is not handled in a standardized way, there are no clear international codes of practice in data management, data access, data querying or data sharing, let alone standardization in algorithms and diagnostic tests. In many instances, but especially when using social media, we have to consider the potential number of duplications of data and control for it. Most probably, data will remain heterogeneous, fragmented, unstandardized, unstructured and unavailable; however, we can develop technological solutions to overcome most of these challenges.

Even though there have been large technological advances in the collection, storage, combination, processing and analysis of data, there are further challenges that remain to be solved. When performing data analysis and making conclusions that inform decisions and policy making it is essential to ensure we are not offering false positive evidence and we are quantifying the likelihood of errors. Adequate statistical methods that control for errors, such as the familywise errors, still have to be improved and implemented as standard practice [Jordan and Mitchell 2015]. Another example is pathogenomics, where whole genome sequencing of pathogens could disrupt dramatically microbiological diagnostics. Many academic systems have shown the benefit to adopt next generation sequencing in the determination of pathogens, their strains and resistances [Wyres et al. 2014]. But it requires industry standard technology to scale out such systems across public and clinical 
pathology laboratories including process management, record keeping and tracking of samples and algorithms so that the analysis can also stand in court if need be [Wyres et al. 2014].

An additional aspect that the community has to address and policy makers need to tackle today is the main ethical challenge of personal privacy. It is one of the main challenges in the use and adoption of big data for healthcare applications. The importance to preserve data sharing and openness should never come at the expense of personal privacy. In addition to the obvious precautions against hacking, all stakeholders should ensure that none of the big data uses lead to corruption and the unethical use of personal data (e.g. clinical trial data or data from EHRs). This point is especially important due to the specific nature of private medical and genetic information, which constitutes a large section of the data analysed for health purposes. Data ownership is another ethical challenge, however it has the potential to become an opportunity if it is used and implemented in a socially acceptable manner [Hafen, et al. 2014]. For big data to succeed in health care we need to create good policies, which are especially important to govern our 'knowledge commons' and 'data commons' [Frischmann et al. 2014]. The General Data Protection Regulation, currently debated at the European Council and Parliament [European Commission 2015], will have profound implications for the use of big data in and for healthcare.

The difference in competences between stakeholders represents a further big challenge. As one example, physicians and patients will not have the same knowledge as data analysts to understand the data that is being presented to them, for this it is essential to create useful interfaces that present the data in a simple and useful manner for the specific purpose it has been analysed. And even if the data is presented in a simple manner, some stakeholders may need additional support to interpret the data and take the right decisions based on this data. This is especially important when presenting patients with associations between genetic data and their possible outcomes, or policy makers with epidemiological data and the possible consequences.

\section{Conclusion}

The impact of big data in drug development, public health and personal health and care is undeniable. Despite the challenge of heterogeneous, fragmented data that is rapidly increasing in volume at high speed; big data analytics making data transparent, easily accessible and actionable becomes a crucial aspect in realizing the potential. Turning data into information and knowledge requires advanced analytical, cognitive computing tools, intuitive visualization and visual analytics so that all stakeholders in drug development and public health can access the right 
data at the right time at the right place to take evidence-informed decisions that lead not only to an improvement in health outcomes but also to a more effective and efficient health system. It remains very clear though that the big challenges have a nature of equal variety as big data itself: Fragmented data sources, no standardization of data models and storage, analytical pitfalls in statistical methods, record keeping and process management, patient privacy and private interest versus the common good.

In order to overcome these challenges we need good policies and to align the interests of all stakeholders involved in health care: Patients, regulators, providers, manufacturers, payers, decision makers and researchers, among others, need to understand the need to maximise the potential of big data and 'data commons' in health and follow the same ethical principles when doing so. 


\section{References}

Anderson, S, et al. "Sequence and organization of the human mitochondrial genome." Nature, 1981: 290: 457-465.

Belle, A, R Thiagarajan, SM Soroushmehr, F Navidi, DA Beard, and K Najarian. "Big Data Analytics in Healthcare." BioMed Research International, 2015: 2015:370194.

Bhatt, Arun. "Evolution of Clinical Research: A History Before and Beyond James Lind." Perspect Clin Res, 2010.

Boyle, J. "Biology must develop its own big-data systems." Nature, 2013: 499(7456):7.

Broniatowski, DA, MJ Paul, and Dredze M. "Twitter: big data opportunities." Science, 2014: 345(6193):148.

Chiolero, A. "Big data in epidemiology: too big to fail?" Epidemiology, 2013: 24(6):938-9.

Davies M, von Cavallar S, Wyres KL, Reumann M, Sepulveda M, Rogers P. "Intuitive information and knowledge representation of disease incidence and respective intervention strategies". Studies in Health Technology and Informatics. 2014;205:1173-1177 DOI 10.3233/978-1-61499-432-9-1173

European Commission. http://ec.europa.eu. 2015. http://ec.europa.eu/justice/data-protection/review/ index_en.htm (accessed August 12, 2015).

European Health Forum Gastein. Big data workshop. Workshop report, Gastein: European Health Forum Gastein, 2013.

FDA. open.fda.gov. 2014. https://open.fda.gov/drug/event/ (accessed August 12, 2015).

Feldman, S, C Burghard, J Hanover, and D Schubmehl. "Unlocking the Power of Unstructured Data." IDC Health Insights, June 2012: \#HI235064:1-10.

Frischmann BM, Madison MJ, and Strandburg KJ. “Governing Knowledge Commons”. Oxford University Press. 2014

Garrett, A, M O'Kelly, D Walp, and NS Berry. "Lifecycle Modeling and Simulation in Clinical Trials." Applied Clinical Trials, June 01, 2015: 24 (6).

Gotz D, Wang F, Perer A. "A methodology for interactive mining and visual analysis of clinical event patterns using electronic health record data". Journal of Biomedical Informatics. 2014;48:148-159

Hafen, E, D Kossmann, and A Brand. "Health data cooperatives - citizen empowerment." Methods of Information in Medicine, 2014: 53(2):82-6.

Herasevich V., Pieper M.S., Pulido J., Gajic O. "Enrollment into a time sensitive clinical study in the critical care setting: results from computerized septic shock sniffer implementation". Journal of the American Medical Informatics Association. 2011; 18(5): 639-644

Horgan D, Jansen M, Leyens L, Lal JA, Sudbrack R, Hackenitz E, Bußhoff U, Ballensiefen W, Brand A. An Index of Barriers for the Implementation of Personalised Medicine and Pharmacogenomics in Europe. Public Health Genomics. 2014;17:287-298.

Huang Z, Dong W, Ji L, Gan C, Lu X, Duan H. “Discovery of clinical pathway patterns from event logs using probabilistic topic models". Journal of Biomedical Informatics. 2014;47:39-57

IBM. "Mayo Clinic and IBM Task Watson to Improve Clinical Trial Research." http://www-03.ibm.com/. September 08, 2014. http://www-03.ibm.com/press/us/en/pressrelease/44754.wss (accessed August 12, 2015).

Jalali, A, OA Olabode, and CM Bell. "Leveraging Cloud Computing to Address Public Health Disparities: An Analysis of the SPHPS." Online Journal of Public Health Information, 2012: 4(3).

Jordan, MI, and TM Mitchell. "Machine learning: Trends, perspectives, and prospects." Science, 2015 : 349(6245):255-60.

Köpcke F, Prokosch H-U. "Employing Computers for the Recruitment into Clinical Trials: A Comprehensive Systematic Review". Journal of Medical Internet Research. 2014; 16(7): e161

Lehrach H. "Virtual Clinical Trials, an Essential Step in Increasing the Effectiveness of the Drug Development Process". Public health genomics 2015; 18(6): 366-371

Leyens L., Brand A. "Early Patient Access to Medicines: Health Technology Assessment Bodies need to catch up with new Marketing Authorization methods". Public Health Genomics. 2016; 19:187-191 
Leyens, L, Richer, E, Mellien, Ø, Ballensiefen, W, Brand, A. “Available Tools to Facilitate Early Patient Access to Medicines in the EU and the USA: Analysis of Conditional Approvals and the Implications for Personalized Medicine". Publich Health Genomics. 2015: DOI: 10.1159/000437137 (in press)

Martin-sanchez, F, and K Verspoor. "Big data in medicine is driving big change." Yearbook of medical informatics, 2014: 9: 14-20.

Milinovich GJ, Soares Magalhaes RJ, Hu W. "Role of big data in the early detection of ebola and other emerging infectious diseases". Lancet. 2015;3:e20-e21

Munoz, J, and R Kurzrock. "Targeted therapy in rare cancers-adopting the orphans." Nature Reviews Clinical Oncology. 2012;9, no. 631-642.

Nature. "The big challenges of big data." Nature, 2013: 498:255.

O’Donnovan J, Bersin A. “Controlling Ebola through mHealth strategies”. Lancet. 2015;3:e22

Ola O., Sedig K. "The Challenge of Big Data in Public Health: An Opportunity for Visual Analytics". Online Journal of Public Health Informatics. 2014;5(3):e223

PatientsLikeMe. "http://blog.patientslikeme.com." PatientsLikeMe and the FDA Sign Research Collaboration Agreement. 15 June, 2015. http://blog.patientslikeme.com/2015/06/15/patients likeme-and-the-fda-sign-research-collaboration-agreement/ (accessed August 12, 2015).

Perer A, Wang F, Hu J. "Mining and exploring care pathways from electronic medical records with visual analytics". Journal of Biomedical Informatics. 2015;56:369-378

PerMed 2020, CSA. The PerMed SRIA: 'Shaping Europe's Vision for Personalised Medicine'. Koeln (Cologne): German Aerospace Center (DLR), 2015.

Raghupathi, W, and V Raghupathi. "Big data analytics in healthcare: promise and potential." Health Information Science and Systems, 2014: 2:3.

Sage Bionetworks. www.synapse.org. 2015. https://www.synapse.org/\#!Synapse:syn2580853/ (accessed August 12, 2015).

Sanger, F, and AR Coulson. "A rapid method for determining sequences in DNA by primed synthesis with DNA polymerase." Journal of Molecular Biology, 1975: 94(3):441-8.

Science Europe. How to Transform Big Data into Better Health: Envisioning a Health Big Data Ecosystem for Advancing Biomedical Research and Improving Health Outcomes in Europe. Workshop report, Brussels: Science Europe, 2014.

Sun J, Wang F, Hu J, Edabollahi S. ACM SIGKDD Explorations. 2012;14(1):16-24

Szezák, N, M Evers, J Wang, and L Pérez. "The role of big data and advanced analytics in drug discovery, development, and commercialization." Clinical Pharmacology and Therapeutics, 2014.

The Economist. "Medicine by numbers." The Economics Technology Quarterly, March 7, 2015: 19-20.

Wang, LW, AP Qu, JP Yuan, C Chen, SR Sun, and MB Hu. "Computer-based image studies on tumor nests mathematical features of breast cancer and their clinical prognostic value." PLoS One, 2013: 8(12): e82314.

Wetterstrand, KA. "DNA Sequencing Costs: Data from the NHGRI Genome Sequencing Program (GSP) Available at: www.genome.gov/sequencingcosts. ." 2015. http://www.genome.gov/sequencingcosts/ (accessed August 12, 2015).

Wyres KL, Conway TC, Garg S, Queiroz C, Reumann M, Hogg G, Holt K, Rusu LI. WGS Analysis and Interpretation in Clinical and Public Health Microbiology Laboratories: What are the Requirements and how do Existing Tools Compare? Pathogens. 2014;3(2):437-458

Zhang S, Li L, Yu Y, Sun X, Xu L, Zhao W, Teng X, Pan Y. “A novel approach to generating CER hypotheses based on mining clinical data". Studies in Health Technology and Informatics. 2013;192:991. 

Chapter 7

Tools from other scientific fields:

game theory 

All disciplines and industries have adopted different tools in their routine work. Healthcare, economics, public policy or metallurgy may use very different mechanisms to solve similar problems. There is big potential in evaluating the tools used in other domains, look for similarities and analyse the possibility to apply them in drug development and healthcare. This section evaluates game theory as a decision supporting tool in drug development and healthcare. Game theory is used in economics and behavioural sciences, which has been recently increasingly applied to other fields.

\section{Game Theory as a potential decision supporting tool: a framework for drug development}

Game theory analyses the possible strategies in competitive situations for independent competing actors, where the outcome of a participant's choice depends on the actions of other participants. Therefore, it can be considered as the science of strategy. Game theory helps to model situations and suggests best outcomes for the involved stakeholders. The essential components in a game are: the players (or decision makers), the actions they can do, the payoffs they get (utility function) and their strategy or choice of action. Game Theory assumes that players are rational and act in their own self-interest; and that the outcome of the game depends on their choices, even though individual players can only control part of the outcome (Leyton-Brown \& Shoham, 2008).

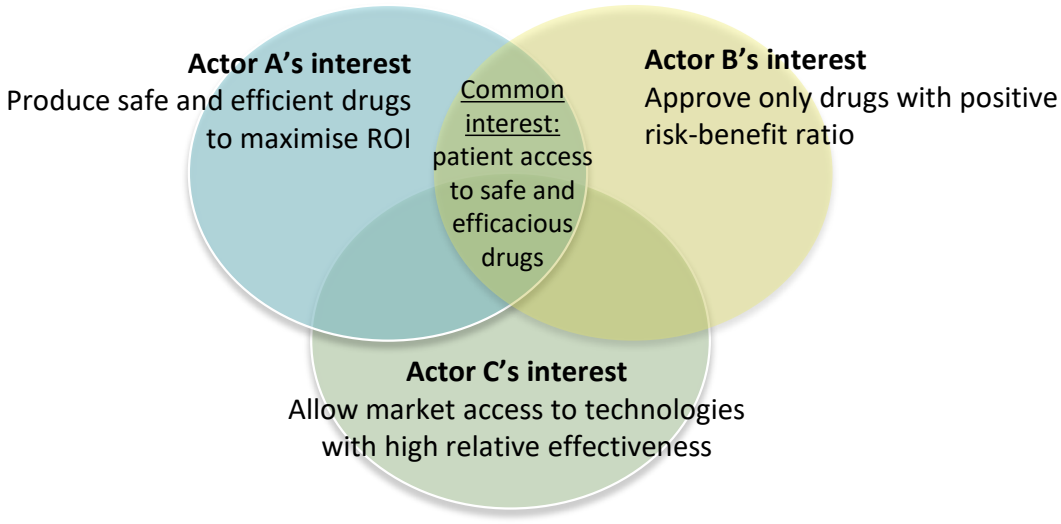

Figure 7-1. non-zero sum game in drug development.

Actor A: drug developers and manufacturers; Actor B: competent authorities; and actor C: payers and health technology assessment bodies. The main interest for each stakeholder is described in the circles, and in the centre, the common interest to all stakeholders is depicted.

Economics is one of the fields where game theory is applied. Depending on the application, different types of games have been described. Zero-sum games represent situations where no wealth is created or destroyed; therefore, players do 
not share a common interest because what one player wins the other one loses. These games can assume perfect information or imperfect information. However, they do not represent the 'real-World' since there is not always a perfect solution for a game. Non-zero-sum games better describe situations we encounter in our everyday life. These games do not have predictable outcomes, or single optimal strategies and are not strictly competitive but also include cooperative elements. Some standard non-zero-sum games have been described that can be applied to many real life situations, such as the 'Prisoner's dilemma', the 'battle of the sexes', the 'Chicken and Volunteer's dilemma', 'Deadlock' and 'Stag Hunt's dilemma' (Shoham \& Leyton-Brown, 2009).

For our purpose non-zero-sum games where imperfect information is described will be considered; in Figure 7-1 presents the actors (drug developers and manufacturers; competent authorities; and payers and health technology assessment bodies) and their individual principal interests, including the common interest of facilitating patient access to safe and efficacious drugs. In drug development some players may not have full information (e.g. CAs and patients), and others may miss something in the data collected even if they think they do have full information (e.g. manufacturers and applicants). Mores, in personalised medicine and rare conditions with small patient populations, imperfect information is even more relevant since the uncertainty in the evidence gathered during the development phase is higher. Imperfect information is inherent to the CTA, MAA and HTA processes. At the developer level, it may be deliberate if the applicant shortens the development process or hides negative data, or unintentional if certain safety or efficacy aspects have not surfaced during the R\&D process. This chapter also focuses on extensive form games that include the time component ${ }^{13}$; they are represented as trees and involve sequential moves, keeping track of players' knowledge at each decision node (Figure 7-2).

Game Theory can be used as a tool to explain the decisions and actions of all players in the drug development process and could be potentially used as a decision supporting tool to help the implementation of personalised medicine and other therapeutics. Surprisingly, its application in this field has been limited, with limited literature on treatment planning, drug cocktail selection, the gaming of pharmaceutical patents and the game theoretical decisions of pharmaceutical companies (Sandholm, Jan 2015), (Langmead \& Kammichetty, 2012) (kamichetty, 2011) (Orlando, Gatenby, \& Brown, 2012). Below the actors involved are described, their possible interests / payoffs and their strategies. We concentrate on the European landscape, on the current development process ${ }^{14}$ and on players

\footnotetext{
${ }^{13}$ Versus normal form games, which only consider one point in time and simultaneous moves by all players, with payoffs as functions of actions.

${ }^{14}$ If not specified otherwise, this chapter focuses on standard development approaches and do not consider the flexible regulatory approaches. Nevertheless, the example provided below does focus on CMA and flexible approaches.
} 
involved in the development and market access of new active substances and first in class products, leaving to the side the so called "me too products", generics and bio-similars. Furthermore, an example of the application in the regulatory process is also presented, where the developer is confronted with the option to follow the CMA path or the MAA path. Chapter 7 can serve as the basis for future research into this promising application for game theory.

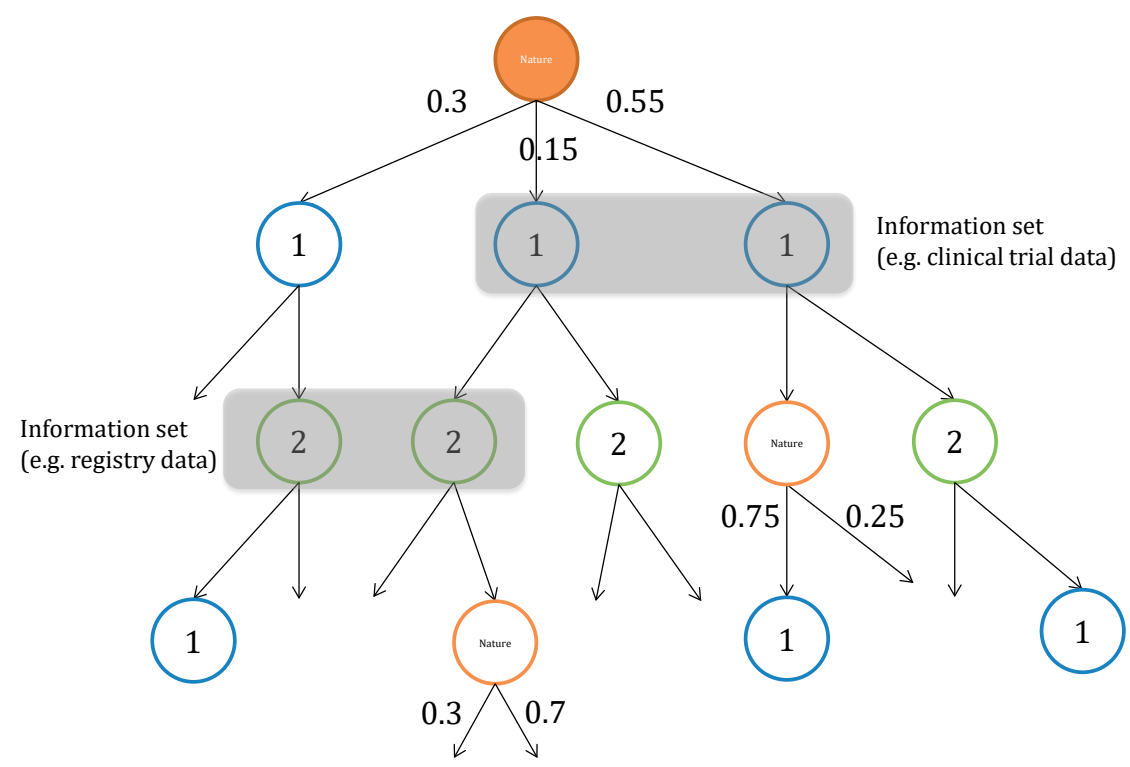

Figure 7-2. Example of an extensive form game in drug development.

Each node is designated with the player whose turn it is to move (stakeholder 1 (the developer) and stakeholder 2 (the decision maker)), who take decisions at each node. At the first set of nodes the developer receives the analysis of the clinical trial results (information set, pictured as a grey bar also denote incomplete information) and takes a decision: continue the development and do a further CTA, stop the development of the drug candidate or go for MAA with the available data. This influences the decision taken by stakeholder 2: approve or not approve the new clinical trial, give or not the marketing authorization, and so on. The orange circles designate "Nature", which introduces stochasticity into the game. Nature is not the disease, it introduces stochasticity on the clinical trial results, patients'state, etc. Modified for drug development from (Sandholm, Jan 2015).

\section{The players: their role}

In order to define the players involved in the game of drug development and market access this chapter divides the development process into 3 phases (see Table 7-1):

1) Research \& Development (including pre-clinical testing and clinical trials),

2) Market Authorization Application (benefit/risk evaluation), and

3) Market Access (pricing and reimbursement). 
Below the players, their actions and their interests (or payoffs) are defined, merging those with the same interests.

Table 7-1. Players in the game of drug development from bench to bed.

\begin{tabular}{|c|c|c|c|}
\hline & $\mathrm{R} \& \mathrm{D}$ & MAA & Market Access \\
\hline Developers & $\begin{array}{l}\text { Researchers, Industry, CROs, } \\
\text { Investors }\end{array}$ & Applicant 15 & Applicant ${ }^{15}$ \\
\hline Decision makers & $\begin{array}{l}\text { Ethic Committees }{ }^{16} \text {, } \\
\text { CAs in CTA } \\
\text { CA in early dialogue }\end{array}$ & $\begin{array}{l}\text { CA (national and supra- } \\
\text { national) }\end{array}$ & $\begin{array}{l}\text { HTAB, reimbursement } \\
\text { agencies, } \\
\text { Public health professionals }\end{array}$ \\
\hline Payers & & $\begin{array}{l}\text { (National health systems, } \\
\text { insurances }{ }^{17} \text { ) }\end{array}$ & $\begin{array}{l}\text { National health systems, } \\
\text { insurances }\end{array}$ \\
\hline End users & Patients, citizens, HCPs & Patients, citizens, HCPs & $\begin{array}{l}\text { Patients, citizens, HCPs, } \\
\text { NGOs }\end{array}$ \\
\hline
\end{tabular}

Divided in drug development phases and in interest groups (grouped into the categories listed in the first column). CA: Competent Authority; CTA: Clinical Trial Application; HCPs: Healthcare professionals; HTAB: Health Technology Assessment Bodies; MAA: Marketing Authorization Application; NGO: Nongovernmental organization R\&D: Research and Development.

\section{Researchers}

Researchers from academia and private companies, lead the initial stages of development of medicines and therapeutic technologies. The development starts with basic research in laboratories, with proof of concepts in vitro tests and early in vivo studies in small animals. The researchers are responsible for developing drugs that are potentially efficient for human conditions with an acceptable risk profile given the characteristics of the disease. Researchers can understand the possible toxicities of the drug through in-vitro and in-vivo pre-clinical models. This data casts light on the safeguards that have to be implemented to administer the medicine to humans to avoid certain risks.

Researchers act in the following interests: the ethical reward of developing technologies to cure diseases; the scientific reward of advancing science, obtaining patents; the personal reward of being recognised for developing successful technologies; and the monetary reward of income (in academia or in private companies) or from licensing the discovery to other companies.

\footnotetext{
15 the term applicant includes: pharmaceutical and biopharmaceutical industry, manufacturers and academia

16 ethics committees are included as decision makers since they decide on the conduct of clinical trials and can approve or reject a trial. This is country specific and does not apply to all countries, it depends on the national or regional clinical trial regulation.

17 this may start earlier during the MAA process or R\&D if there is an early access scheme for the product
} 


\section{Industry}

Industry (in the form of large international companies, small and medium enterprises or small spin-offs) drives the development of medicines and health technologies from the initial basic research steps or from the time of licensing until market access. They invest heavily in the research and development process, sometimes requiring the money from external investors. Medicines are tested step-wise to elucidate its characteristics and safety profile in well-defined in vitro and in vivo models. Once the initial proof of concept is established in animals and the required safety profile has been elucidated, the developer evaluates whether the drug candidate is adequate for human use depending on the pre-clinical data. The drug is then tested in humans in clinical trials if the company decides to continue with the development. Here the company also acts as the sponsor of the trial and is responsible for the regulatory approval (Clinical Trial Application or CTA) of the trial and for its ethical and qualitative conduct. Developers may outsource and delegate some of their activities to Contract Research Organizations (CROs) who take over the activities of the sponsor, especially in relation to clinical trials. However, the sponsor remains responsible for the oversight and conduct of the trials.

The company proceeds with the Marketing Authorization Application (MAA) process if it considers the data from pre-clinical and clinical trials is satisfactory from a safety and efficacy point of view (risk / benefit). The applicant prepares a dossier with all the data compiled during the research and development phase and files to the relevant competent authorities to seek marketing authorization (MA).

Once MA is granted, the MA holder must complete the last step before the drug reaches the market. The pricing and reimbursement process is different in each European country, some countries have national or regional HTA bodies who require an HTA to accept or reject the proposed price (such as the cost effectiveness analysis requested by NICE in England), or who carry out relative effectiveness analysis and classify the drug in price categories according to preestablished aspects (e.g. innovation, effectiveness, etc.). The applicant prepares a dossier depending on the national pricing and reimbursement process.

Developers are mostly profit making organizations and have therefore strong monetary interests, but they also have other payoffs. Pharmaceutical and biotechnological companies develop medicines with the final goal of marketing the technology, recovering the invested capital and making a profit. In order to maximise their return on investment (ROI), they are interested in reaching the market as soon as possible, and therefore reducing the time of the R\&D process. They also have the moral interest of developing successful medicines to cure diseases or improve the quality of life of patients; and the technological interest of developing innovative products and being recognised for this. 


\section{Decision makers}

Decision makers are the public institutions who review the information provided by the applicants in the CTA, MAA and HTA processes of drug development, and who are responsible for the oversight of clinical trials, the approval of safe and efficient medicines, the access to effective drugs and the control of the medicines industry and market. Their main responsibility is to safeguard patients, citizens and the national health system. To do so they:

- Evaluate the ethical, medical, scientific and legal aspects of CTAs to ensure that only clinical trials that are ethically, medically and scientifically sound with safe and high quality medicines are offered to patients and citizens. Ethic Committees and Competent Authorities share the responsibility during the CTA process, each country regulates nationally the responsibilities of each body.

- Assess the risk / benefit ratio during the MAA process. The competent authority (usually national medicines agency, coordinated by the EMA if they follow a centralised approval procedure) evaluates the data provided by the sponsor and any other necessary evidence to determine the risk/benefit ratio of the medicine. The acceptable risk and benefit profile varies depending on the disease. If the ratio is considered positive, marketing authorization is granted and the medicine is considered safe and efficient for the approved indication and population.

- Analyse the effectiveness data provided by the applicant or compiled by the responsible body to determine the price or decide if the drug can be reimbursed in the national, regional or local healthcare system. This pricing and reimbursement process is extremely heterogeneous in Europe, with more than 28 responsible bodies, regulations and methods. In some countries independent HTA bodies (e.g. UK) are responsible for the evaluation and decision, in others the national health authority (e.g. France) or the medicines agency (e.g. Italy) performs the review (European Parliament, 2015). According to the applicable regulations, the responsible body decides if the medicine should access the market, the price it may have or if it is reimbursed through the national healthcare system.

The decision makers described above (ECs, CAs, HTABs and other public bodies) ${ }^{18}$ share the common interest of citizen's safety. They approve safe and ethical clinical trials and maintain the oversight to ensure they are run accordingly. They grant marketing authorization to safe and efficient medicines. And they allow market access to effective medicines. Furthermore, regulatory bodies involved in the pricing and reimbursement process, also have the interest of safeguarding the

${ }^{18}$ Policy makers will not be considered here since they are out of the scope of this thesis. However, they are an important actor for the implementation of personalised medicine one step beyond market access. 
healthcare system's sustainability. In order to do so, they do not allow unreasonable high prices or ineffective drugs into the market that may drain the available resources and deny effective treatment to other patients. There may be vested interests, economic or personal, in some of the reviewers but these institutions usually have control steps in place to avoid conflicts of interest.

\section{Payers}

Healthcare systems must safeguard their patient-population and citizens. The public health professionals and institutions responsible for running them must ensure all patients have access to equitable, safe and adequate treatments (this is at least the case in the EU). In EU countries, there are at least two types of payers: the national or regional healthcare system and the public or private insurance companies. Mostly they act depending on the conclusion of the HTA body or institution that performs the relative effectiveness evaluation: they may include or exclude the drug from the reimbursed medicines list or negotiate the price with the applicant. They may as well enter into Managed Entry Agreements (MEAs) with the applicant (Ferrario \& Kanavos, 2013) or have other mechanisms to finance the drug (e.g. cancer drug fund in the NHS in England).

The interest of public health systems is to enable access to the best possible treatment in a resource constrained setting. Correct resource allocation is an essential aspect of healthcare systems due to the opportunity costs that are inherently created: to finance the treatment of some patients others may not become theirs. Some payers, such as insurance companies, are profit-making organizations and have strong economic interests, which may interfere with the public health interest to protect the health of the patients they insure.

\section{End users}

The end users of the drug development process are the citizens, including patients and healthcare professionals (HCPs). Patients receive the drugs or therapeutic technologies through their healthcare providers, healthcare professionals and pharmacies. HCPs use the medicines to treat the conditions of their patients according to the approved indication and to the information set in the Summary of Product Characteristics (SmPCs), or sometimes off-label if they perceive a potential benefit for the patient.

The interest of the end users is often personal. Direct or indirect interest moves the actions of citizens and patients, who either suffer a condition themselves or have a relative or friend who suffers it. Patients and relatives want to receive the best treatment that can cure the disease, stop the progression or ease the symptoms, depending on the condition. They often obviate the fact that resources 


\section{Chapter 7}

are scarce and limited. Vocal patient groups, who get more attention from the media and society and therefore receive better more costly treatments, may shift the balance of the usual process that aims to ensure equitable distribution of resources at the expense of other treatments for quieter patient groups.

HCPs have the ethical interest of providing the best treatment to their patients; and an economic interest through their remuneration. The pharmaceutical industry has been heavily regulated to avoid conflict of interest from physicians and ensure they prescribe the best available therapeutic option to their patients. In contrary, the medical device industry, is not well controlled regarding possible monetary conflict of interest.

\section{The players: their strategies (how each players' actions affect the well-being of other players)}

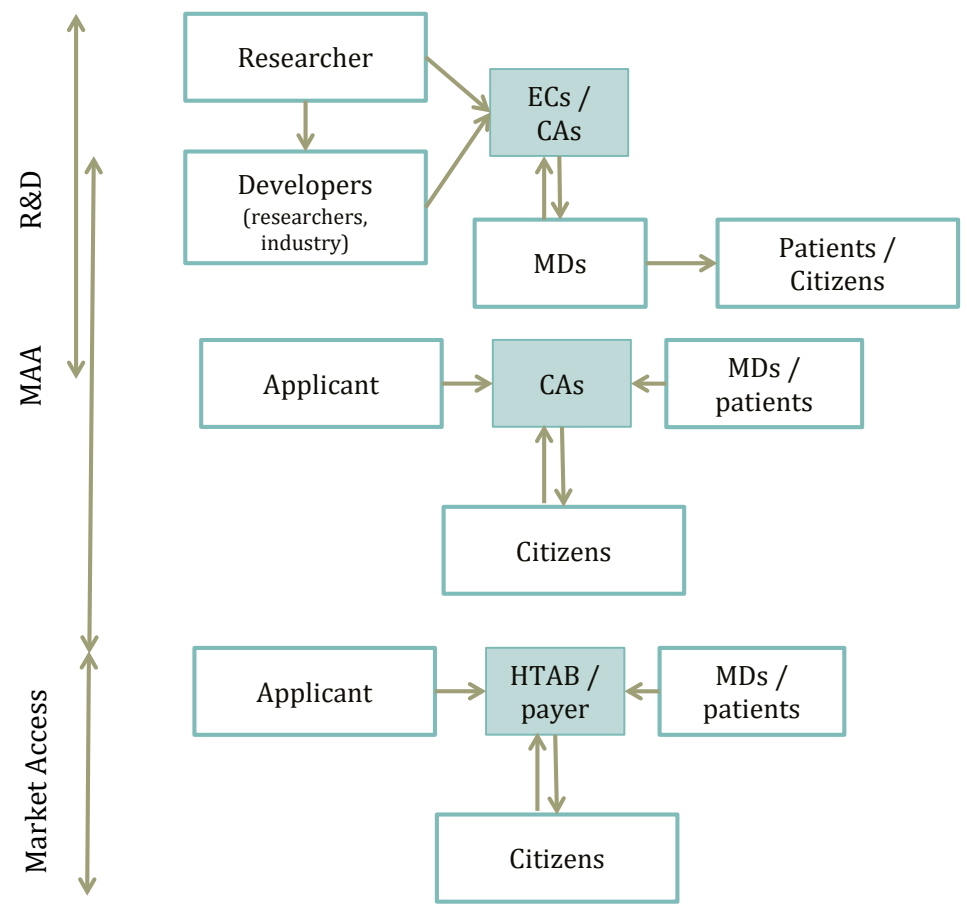

Figure 7-3. Game of drug development. Depicting the interactions of the players, their strategies and how they affect each other.

Figure 7-3 presents the game of drug development described in this section. The concept of MAA as a longer phase of drug development is considered from the point of view of the new flexible approval methods and early dialogue initiatives described in chapters 4 and 5 . This process can start as early as pre-clinical development and is no longer meant to be a specific moment in time or the final 
objective of the drug development process. It should rather be considered as a parallel process that includes early discussions with regulators and other stakeholders. The process has been simplified to concentrate on the main players that are present from the initial R\&D until market access; thus, essential players such as CROs or investors and other players who participate within the development phases are obviated from this image.

Game theory describes the principal-agent relationship, whereby the principal contracts the agent to perform an action on its behalf (Table 7-2 describes the ones in drug development). In such relationships the situation of moral hazard has often been reported, where the agent incurs in risks that may harm the principal because the agent does not suffer the consequences personally. Moral hazard may also arise if the agent receives inadequate incentives to perform according to the contract.

Table 7-2. Principal-Agent relationship in drug development.

\begin{tabular}{lll}
\hline Process & Principal & Agent \\
\hline R\&D basic research & Industry & Researcher \\
R\&D clinical trials & Industry & HCPs and patients / citizens \\
R\&D CTA & Decision makers & Industry (influencing its action) \\
R\&D CTA & Citizens & EC, CA \\
MAA & Decision makers & Industry (influencing its action) \\
MAA & Citizens & CA \\
Market access & Decision makers & Industry (influencing its action) \\
Market access & Citizens & Decision makers and payers \\
\hline
\end{tabular}

CA: Competent Authority; CTA: clinical trial application; EC: Ethic Committee; HCPs: healthcare professionals; MAA: Marketing Authorization Application; R\&D: research and development;.

In the first steps of R\&D, researchers perform the development tasks for the pharmaceutical industry or the developers. At the clinical trial stage, industry contracts health care professionals to perform the studies and these in turn engage patients / citizens who enrol into the trials. And finally, ethic committees and competent authorities review and authorise the trials on behalf of its citizens. An example of moral hazard during this step is a health care professional who forges clinical trial data from invented patients in order to increase their remuneration. Patients assume the risk when they receive the drug once it receives MA, because the false data (depending on the extent) may hide side effects or increase the real efficacy; and the developer company may also incur in the risk of being discovered and the drug being penalised or their entire clinical trial structure questioned.

At the MAA step, industry as an agent provides the documentation and data needed for the decision makers to evaluate the drug. And in turn, competent authorities and decision makers are the agents of citizens in the evaluation of marketing authorization application dossiers and deciding which drugs have a 
positive risk/benefit ratio and can reach the patients. A developer company hiding information or providing forged information can incur in moral hazard at this step, decision makers take the risk of making a wrong decision based on wrong data and patients have the risks of receiving a treatment that is riskier or less beneficial than imagined.

During the pricing and reimbursement procedure, industry again acts as the provider of data to the decision makers depending on the local requirements. This data can be relative effectiveness data compared to the current standard of care. And again, decision makers and payers are the agents of citizens and decide what medicines to fund through national health care systems or health insurances depending on the effectiveness of the therapy and the given constrained resources. An example of moral hazard at this stage is a decision maker or payer who takes a specific decision because himself or his family have special interests on the drug or the disease (economical or health interests). Patients with other diseases who are not able to receive the best treatment because of the lack of resources are one example of risk takers in this case.

In summary, the strategy of researchers and developers is to create the necessary information on safety and efficacy of the drug in the minimum amount of time possible to reach the market and start making profits as soon as possible; however, they have to balance this with creating the right data required by the competent authorities to reach a decision on the compound. Health care professionals have to perform clinical trials in the best possible way complying with all legal requirements in order to produce high quality data, to later in time provide the best possible treatments to their patients. Competent authorities need to ensure that all necessary information is available and truthful in order to reach a decision on the benefit/risk ratio of the therapeutic with the least possible uncertainty. And finally, payers must ensure that their patient population receives only the best available treatments with the fewest possible side effects in order to optimize treatment and health care resources.

\section{Application: example of game theory incorporating the CMA and MAA paths}

Below an example of an extensive form game that models the interactions between the involved actors is presented, incorporating the MAA and CMA regulatory path. The nodes describe decision points where new data is available (e.g. from clinical trials) and the decision at the competent authority level with the submission dossier or as a result of early dialogue discussions. The first steps up to conditional marketing authorization application are depicted in Figure 7-4 and the following steps (including the P\&R process) are depicted Figure 7-5. 
For a pharmaceutical company the time between start of development and reaching the market is resource intensive, therefore more time to create better information translates into an increased use of resources. Fast entry into the market means higher ROI and maximises utility. However, an increased investment in research would also improve the quality of information and might make the regulatory review process faster.

The competent authority is interested in the quality of information. The resources spent by the developer in the R\&D process are not explicitly relevant ${ }^{19}$, and preferably more information should be sent than less. If the quality of information is not sufficient the developer will need to respond many rounds of questions (presented in Figure 7-4 and Figure 7-5 as "+ data") and may need to perform further studies, delaying the entry to the market and losing the time gained by producing less data. Therefore, the developer maximises its utility by optimising the amount and quality of information provided.

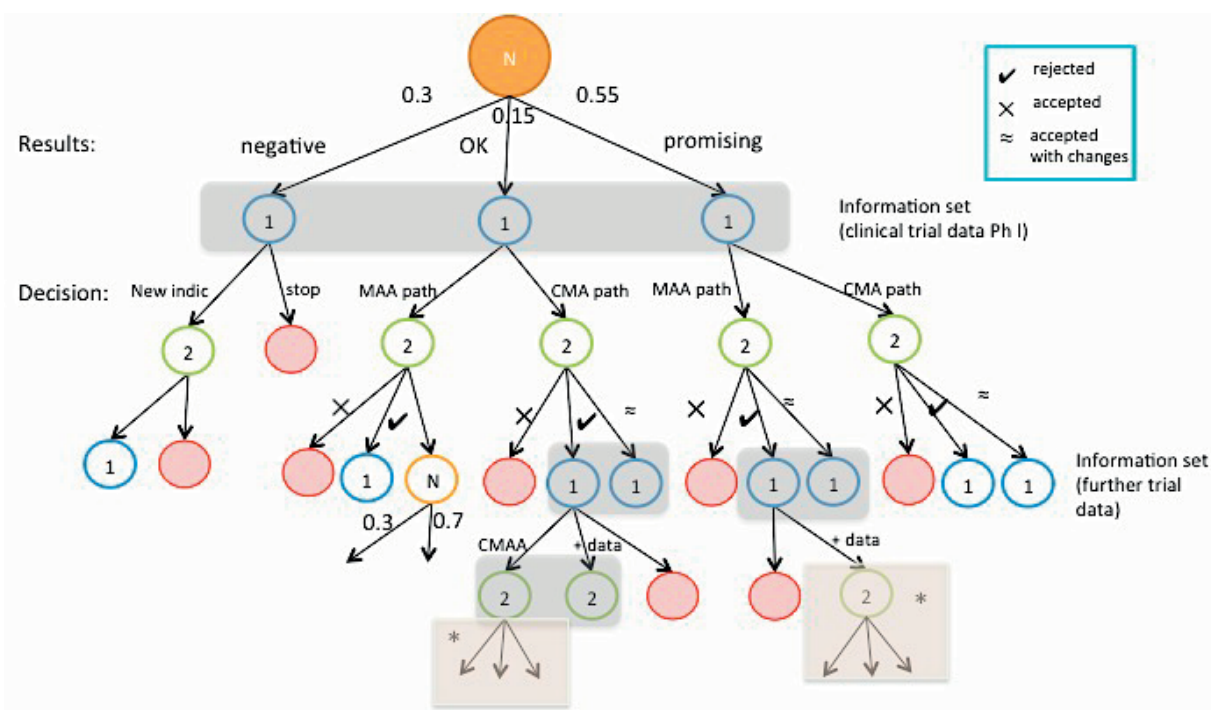

Figure 7-4. Example of game theory model for the interactions between the developer (1) and competent authority (2).

Incorporating the decision between following the MAA path or the CMA path. CMA: Conditional Marketing Authorization; CMAA: conditional marketing authorization application; + data: need for additional data; MAA: Marketing Authorization Application; N: Nature stochastic component. Grey boxes designate information sets, described on the right hand side. The green boxes marked with * designate the image continues from here in Figure 7-5.

As presented in Figure 7-4, stakeholder 1 (the developer) takes a decision at the second node with the information set of the proof of concept phase I clinical trial. If

${ }^{19}$ It is only implicitly relevant, in that often, more resources translate into more data and more information. 
the results are negative, the developer can stop the development or seek another indication, stakeholder 2 (the CA) can either accept the clinical trial application for the new indication or not. If the results of the trial are positive $(\mathrm{OK})$, the developer can decide to continue development within the CMA path or the MAA path. At that point the developer may incur in early dialogue with the CA to discuss the development plan and the CA either accepts the plan, rejects it or requests changes. From now on, we follow the paths where the CA accepts the development plan for MAA and CMA. At the next decision node, the developer evaluates the information set from another clinical trial (planned following the feedback from the CA). In the CMA path, if the data is promising the developer can file the CMA application; if the results are negative he can decide to stop development and if the result is inconclusive, he can perform another clinical trial to gain more data (depicted as "+ data"). Whereas, in the MAA path, if the results are negative he can decide to stop development, otherwise the next clinical trial is designed depending on the results obtained (designated as “+ data").

We now move to Figure 7-5, the CMA path is described in part A): When the CMA application is filed, the CA reviews the evidence provided and takes a decision based on the information set submitted (application dossier). If the benefit / risk ratio is positive and the CMA is granted, the developer will decide how to proceed with the P\&R process, either he submits a dossier to the HTA body or applies for a different route of reimbursement (e.g. cancer drug fund in the UK). The HTA body can decide to deny market access, to grant market access ${ }^{20}$ or to request further evidence or negotiate the conditions -such as the price. Once the medicine reaches the market, HCPs can decide to prescribe the drug to their patients or not. In this example, the probability that a doctor prescribes a conditionally approved medicine is described as low (30\%) because HCPs are still reluctant to believe that the positive risk / benefit ratio has been established by the CA. If the medicine, however, is the only option for their patients then the probability would be much higher.

The final decision whether to take the drug or not lies with the patient (when the patient is able to decide), and depending on this decision the final utilities can be assigned to the stakeholders: the developer receives positive utility if the patient receives the drug, the patient gains positive utility depending on the effect of the drug and its side effects on the disease and her quality of life, the payer receives a mix of negative monetary utility and positive utility depending on the effect of the drug on the patient, her quality of life, the quality of life of her carers, and other societal utilities. Societal benefits are really varied (e.g. going back to work, not needing a carer, increased quality of life of carers and family members, etc.), there are good reviews in the field of health economics on this aspect (Sussex, Towse, \&

\footnotetext{
20 The model has been simplified in the aspect of market access, where the large variations in national market access and price negotiations procedures are not considered.
} 
Devlin, 2013) and further discussion are out of the scope of this work. If the CMA is not granted and further information is requested, the developer will need to perform further analysis or further clinical trials. He can also decide to stop development at this point in time. When additional evidence is presented, the CA has again the option to grant CMA, deny CMA or request further information. If CMA is granted the P\&R process described above is repeated, with the delay in time from the additional round of questions and the consequent reduction in final utility from a delayed market entry.

In the MAA path depicted in part B), as presented in Figure 7-4, more data was produced by the applicant, the decision of the competent authority at this top node, can be either to decide on a CTA or to respond to an early dialogue request (or scientific advice). The CA can reject the proposal, request changes or accept it. Next, with the information gained from the new clinical trial the developer can decide to file the MAA, get more evidence from a further clinical trial or stop the development. If MAA is filed, the CA has the option to grant the marketing authorization (MA), request further information or deny MA. From this step on we can go to the description of the possibilities described from CMA in figure part A). Once the medicine reaches the market, HCPs may be more inclined to prescribe the drug because it has full market authorization, this would change the probabilities and affect the final utility. For the final utilities, it is important to note that under CMA the patient population is per definition smaller and the drug would reach a smaller market, whereas under full MA, the target patient population is larger.

CMA may not be the most appropriate option for many drugs, in cases where reaching early the market means that HCPs and patients will not trust the validity of the CA's decision and will not use the medicine even if the results are good; it might be better to wait for evidence to present full MAA and reach a wider patient population. Game theory can help identify these instances and avoid a failed market entry due to CMA, or contrarily avoid the late market entry under MAA if CMA could maximise utilities.

The probabilities assigned to each node depend on the molecule under development and could be introduced by each developer when using the model. As already described above, players want to maximise their utility (or pay-off) with their actions, and this exercise can help in the decision making process to take the best route given the information available.

A large limitation with this model is that it only includes the steps up to the first authorization; therefore, in the CMA path the final submission of evidence in order to convert the licence into a full MA is not considered. However, this could be easily designed if the model should be used in real practice. Furthermore, the model has been simplified in some aspects, such as market access, where the large variations in national market access and price negotiations procedures are not considered; or by obviating the decision nodes between clinical trial results. Depending on the 


\section{Chapter 7}

decision that needs to be reached, more granularity of the process needs to be introduced in the model. This model reflects the decision between CMA or MAA for a given drug and therefore less detail is acceptable.
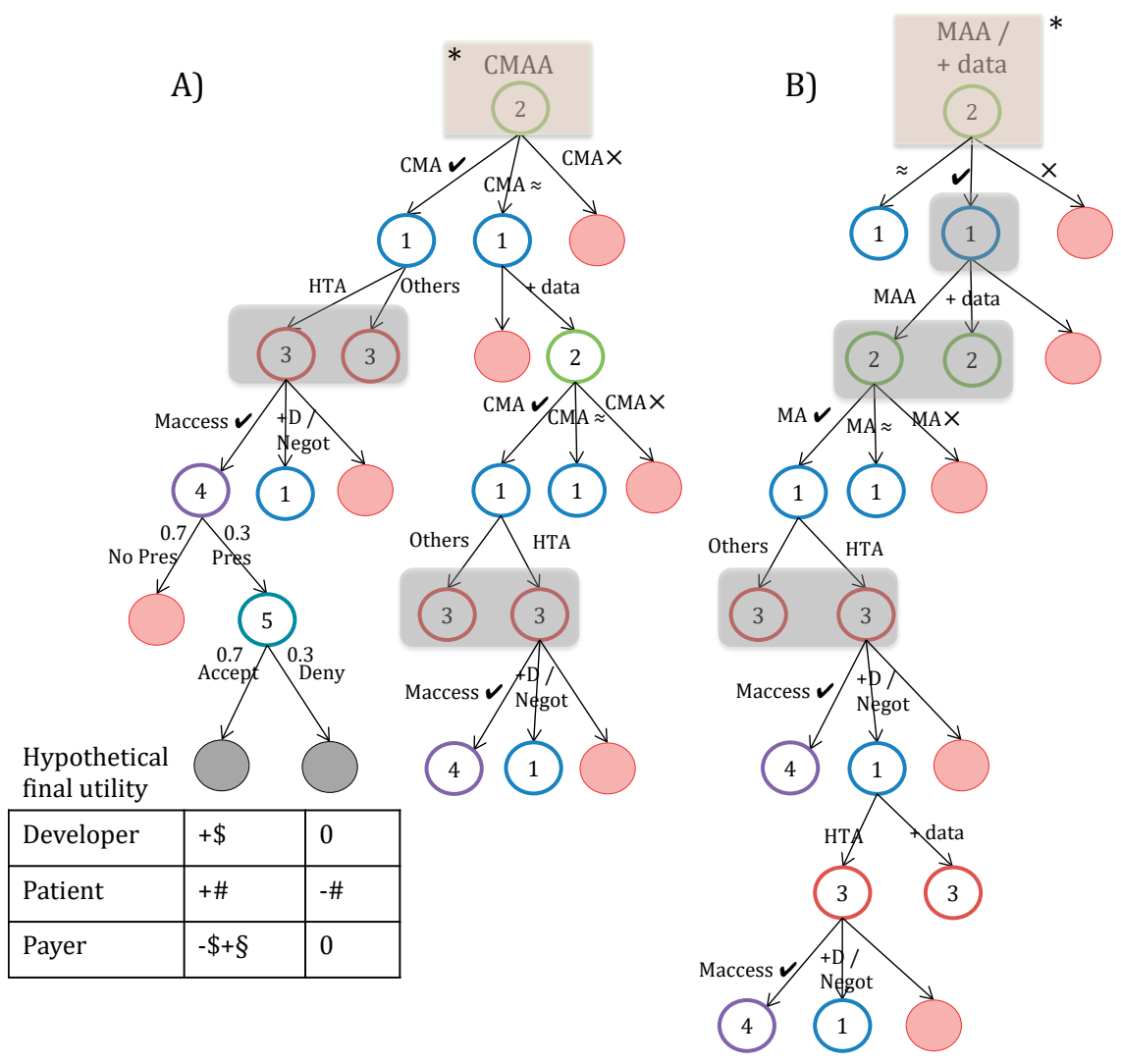

Figure 7-5. continuation of Figure 7-4.

A) CMA path and B) MAA path. Here the process of marketing authorization application, pricing and reimbursement and market access are depicted. 3 further stakeholders are added: HTA bodies (3), HCPs (4) and patients (5). CMA: Conditional Marketing Authorization; CMAA: conditional marketing authorization application; + data / +D: need for additional data; HTA: health technology assessment; MAA: Marketing Authorization Application; MA: marketing authorization; MAccess: market access; N: Nature; Negot: negotiations; no pres: no prescription from HCPs; others: other pricing mechanisms such as cancer drug fund; Pres: prescription to patients by the HCP. Grey boxes designate information sets: HTA submission dossier. The green boxes marked with * designate the image continues from image 9. Black circles indicate the end of the tree. Hypothetical final pay-offs: \$: Monetary pay-off, positive for developer and negative for payer. \#: the pay-off for the patient depends on the effect of the medicine on the disease and the general quality of life, and the possible side effects. The patient may get a negative payoff from not accepting to take the medication if the medication would have had a positive effect on the disease and the patient's quality of life. §: the payer has a direct negative monetary pay-off on the price of the medicine; however, there is an indirect positive societal pay-off related to the improvement of the quality of life of the patient (if any improvement). 
Another interesting application of game theory could be to model the outcomes of MEAs and see if the stakeholders should invest time in engaging in a MEA or if standard pricing methods are better. This could be modelled similarly to the example above and the pay-offs could be very different and useful for payers and developers to see if the resources invested in negotiating and managing the MEA are worth it compared to the pay-offs received. For the payer all pay-offs should be considered, including societal benefits, which would make the equation difficult to calculate. However, once a standard method has been developed for this pay-off calculation the model could be very useful for all stakeholders.

\section{Risk and uncertainties}

Many uncertainties and challenges arise in the application of game theory to the drug development process. The pharmaceutical market is an inherent imperfect market since there is never $100 \%$ certainty about the factors that influence the market. Some aspects that bring uncertainties and constitute limitations on the use of game theory in the drug development process are:

- The diseases themselves, each condition is different in each patient due to the environment, co-morbidities, concomitant medications and other factors. It is therefore difficult to assign probabilities and utilities to the different strategies.

- Patients do not act rationally, very often patients do not act in their own interest. If the example of patient adherence is considered, effective adherence averages only 50\% when considering all factors involved (Christiansen, 2004), but it would be rational to adhere to the medication in order to maximise the utility gained from it. This affects tremendously the pay-off for patients and society and can only be influenced by the developer or other stakeholders to a certain extent (Conn, Ruppar, Enriquez, \& Cooper, 2016). Game Theory assumes that all actors act rationally and therefore these un-rational actions increase the uncertainties of the model.

- Unknown risks lie in the rare safety findings that cannot be elucidated in clinical trials with small populations, these affect the effective pay-off of patients and society. But it can also affect the pay-off of the developer if there is a MEA or if a licence is finally revoked due to these safety aspects. It is difficult to include this uncertainty into game theory models; however, a standard probability could be included if a systematic review is able to find the average probability of such rare findings.

- The regulatory process can add uncertainty to the model in that it does not provide predictable pathways for all types of new technologies (nanomedicines, organ printing, ATMP, etc.). However, in certain cases, as in nanotechnologies it is difficult to create guidelines that cover all aspects of all 


\section{Chapter 7}

possible products. This would entail additional rounds of questions by the decision makers and additional data requested to the developer which delays market access and affects the pay-offs of all stakeholders.

- Lobbying from strong patient organizations may shift certain decisions, especially in the $\mathrm{P} \& \mathrm{R}$ process, towards providing patient access to medicines that do not have a convincing evidence base compared to others.

- The performance of a promising new drug during the development phase in clinical trials that are conducted in ideal situations may be different to the performance in the market on patients with poly-medicines and co-morbidities. This would affect the pay-off of the patient and the other stakeholders if there are MEAs and if the license is revoked. It is especially relevant for the use of RWE, which would capture this safety and efficacy in "real-World patients".

- We must also consider the uncertainties that asymmetric information brings into the game, some have already been discussed above. At least two types of effects on the model can be described:

o Adverse selection model $\rightarrow$ as described in the finance and insurance market, some stakeholders may have more information than others before the action is completed. If, for example, developers hide safety information at the time of MAA they have an advantage of information and the decision of the competent authority will be biased without the full information set. This includes a lot of uncertainties to the model, since when the medicine will be used in the market the safety signals may appear. This affects the pay-offs of all stakeholders, more negatively of patients who suffer the consequences of the safety signals.

o Moral hazard model: as described above, in a principal-agent relationship, agents may act in their own interest and negatively affect the interest of the principal. This can affect the utility of all players and introduces uncertainties into the model.

Furthermore, the new developments at CAs (as described in chapters 4 and 5) will affect the uncertainties in different ways, two examples are:

The adaptive pathways pilot increases the uncertainties in safety and has been often criticized for this. However, different tools, such as early dialogue and controlled market access until the full MA is granted, help to minimise them. This will be the case anyway for most drugs as we move into diseasomes and more divided and characterized sub-conditions with smaller patient populations. These conditions are not large enough to have highly powered phase III clinical trials and create the necessary data to achieve negligible uncertainties in safety. Drug development for orphan diseases has already faced these challenges and could give some insight into mitigating strategies. 
Assessing the risk/benefit of a drug and grating MA with less data increases the uncertainties and risks for patients but it also allows faster access to drugs that may be beneficial. Fast access to promising drugs is specially important in conditions where the acceptable risk may be very high, such as in pancreas cancer where incidence and mortality are nearly identical (Milne, et al., 2013). All these aspects can be modelled in game theory to elucidate the best strategy for all players.

Applying early dialogue tools in drug development limits uncertainty for the developers and the decision makers. Developers are more likely to produce the data needed by CAs during the development process and they are more likely to drop drugs that are not promising at the right time point. Whereas decision makers can foresee future applications and new technologies and improve their resource allocation consequently; furthermore, they can already comment on the development plan and can shape this to their evaluation needs.

Some have expressed the concern of moral hazard in the actions of reviewers at the decision makers if they participate in the early dialogue sessions with developers and then also review their applications. On the one hand, these reviewers are better informed about the product and may be better suited to analysing the data and the application. On the other hand, if this is a concern in the general population separate teams can be created within regulatory agencies to divide the process and ensure that different persons are in charge of these processes.

\section{Further steps}

Above the components of game theory in drug development needed to create a model are laid out, and an example on the application of game theory in the drug development process is presented for the first time. This framework can be used in the future to model the interactions between stakeholders and work as a decision supporting tool. Game theory could inform developers if more investment should be placed on the development of a certain molecule; which of the CMA or MAA pathways are more appropriate or if MEAs are the best option over standard pricing methods, between other promising applications. However, more research is needed and the models need to be developed for all the possibilities of application. 


\section{References}

Christiansen, A. (2004). Patient Adherence to Medical Treatment Regimens. New Haven, CT: Yale University Press.

Conn, V. S., Ruppar, T. M., Enriquez, M., \& Cooper, P. S. (Mar 2016). Patient-Centred Outcomes of Medication adherence Interventions: Systematic Review and analysis. Value in Health, 19 (2), S. 277-285.

European Parliament. (2015). Towards a Harmonised EU Assessment of the Added Therapeutic Value of Medicines. Policy Department A: Economic and Scientific Policy. Brussels: European Union.

Ferrario, A., \& Kanavos, P. (2013). Managed entry agreements for pharmaceuticals: the European experience. Brussels: EMiNet.

kamichetty, H. (2011). Application: Games of molecular conflict. In Structured Probabilistic Models of Proteins accross Spatial and Fitness Landscapes (Bd. Chapter 9, S. 121-127). Carnegie Mellon University, School of Computer Science.

Langmead, C., \& Kammichetty, H. (2012). Patent nr. PCT/US2012/026966. USA.

Leyton-Brown, K., \& Shoham, Y. (2008). Essentials of Game Theory. Morgan and Claypool Publishers.

Milne, R., La Vecchia, R., Van Steen, K., Hahn, S., Buchholz, M., Costello, E., et al. (2013). EU Pancreas: An Integrated European Platform for Pancreas Cancer Research - from Basic Science to Clinical and Public Health Interventions for a Rare Disease. Public Health Genomics, 16 (6), S. 305-312

Orlando, P., Gatenby, R., \& Brown, J. (2012). Cancer treatment as a game: integrating evolutionary game theory into the optimal control of chemotherapy. Physical Biology, 9.

Sandholm, T. (Jan 2015). Steering Evolution strategically: computational game theory and opponent exploitation for treatment planning, drug design and synthetic biology. Proceedings of AAAI Conference on Artificial Intelligence, Senior Member Track, Blue Skies category. USA: Association for the Advancement of Artificial Intelligence.

Shoham, Y., \& Leyton-Brown, K. (2009). Multiagent Systems: Algorithmic, Game-Theoretic, and Logical Foundations. Cambridge University Press.

Sussex, J., Towse, A., \& Devlin, N. (2013, Jan). Operationalizing value-based pricing of medicines: a taxonomy of approaches. Pharmacoeconomics, 31(1), 1-10. 
Chapter 8

General discussion 


\section{Flexible regulatory methods: current European landscape}

Chapters 1-3 set the scene for the development of personalised treatments and analyse the gaps and needs for its wider implementation, as well as the current barriers. Chapter 4 describes the available regulatory tools that aim to facilitate earlier patient access to promising medicines, showing that these tools are already available but not optimally used. Chapter 5 outlines the need to have flexible approaches at the HTA level in order to guarantee early patient access through flexible MAA processes. The last two chapters, focus on tools that can be used for decision making: chapter 6 discusses big data from the perspective of drug development and public health laying out the needs to ensure that it fulfils its promises for healthcare; whereas chapter 7 proposes a framework to apply game theory to drug development.

In order to put into the wider context what has been discussed and presented in the previous chapters, this section focuses on the main potential application of this work, which is a very relevant topic nowadays: flexible regulatory approaches. Two leading projects at the European level are evaluating the feasibility of applying flexible regulatory pathways to approve medicines: the Adaptive Pathways (APs) pilot run by the EMA (European Medicines Agency, July 2016), and the IMI project ADAPT SMART which builds on the knowledge of APs and evaluates Medicines Adaptive Pathways to Patients (MAPPs) (IMI, 2017).

Before discussing these projects in detail it is essential to emphasize that they do not aim to institute new procedures or regulatory approaches, they merely propose to use existing tools and technologies. It is also important to lay out the difference between APs and MAPPs: while APs was a project set by the EMA in order to pilot the approach with industry and other stakeholders and analyse the feasibility; the ADAPT-SMART project funded by IMI is a platform to coordinate the activities of all involved stakeholders and evaluate the applicability and implications of MAPPs as well as what needs to be done in order to roll-out the use of these pathways.

\section{Adaptive pathways}

The EMA started a pilot in 2014 to make better use of existing regulatory tools (e.g. conditional marketing authorization (CMA), set in 2006; and strengthened postmarketing monitoring tools introduced in 2012) for drugs in indications of unmet medical need. The idea was to set an iterative approach to evidence gathering and drug development; to involve other relevant stakeholders such as HTAs; and to include real world data (RWD) in addition to Randomised Controlled Trials (RCTs). The pilot received 62 applications of which 6 advanced to the final stage. In 2016, the final report was published with their experience and feedback from other interest groups (European Medicines Agency, July 2016). 
According to the final report, the project had good experience with the safe-harbour early multistakeholder dialogue and agreement of prospective evidence generation. Which is certainly a big added value of the adaptive pathways project. Furthermore, during the pilot, the regulatory framework demonstrated having the necessary mechanisms to monitor the risks and benefits once the drug enters the market with conditional approval and being robust enough to take action if needed. The most important aspects for the successful application of APs were defined as: diseases with clear actionable end-points for post-authorisation decisions; setting control points along the development pathway to revise the level of evidence; having a controlled market access with prescriptions restricted to the adequate patient population; and allowing for managed entry agreements and entry / exit strategies for the relevant stakeholders (European Medicines Agency, July 2016).

It is important to reinforce that early patient access cannot come to the detriment of the patient; in other words, the safety and quality of drugs should not be compromised by any new regulatory approach or the wider implementation of existing flexible approaches. Competent authorities need to evaluate the risk to the patient and to society, of drugs approved with flexible methods and less data. Specially, we need to understand where the uncertainties lie and how we can minimize them. These questions are part of the ADAPT SMART project.

\section{ADAPT SMART and MAPPS}

ADAPT SMART is an umbrella project that brings together all the relevant stakeholders to discuss the important aspects to implement APs. It is defined as "an enabling platform for the coordination of Medicines Adaptive Pathways to Patients (MAPPs) activities" (IMI, 2017). The project joins the efforts of all relevant stakeholders (academia, patient organisation, regulators, payers and HTA bodies) and aims to facilitate and accelerate the implementation of MAPPs in all future drug development programs.

In a podcast released earlier this year, the project leader Hans-Georg Eichler (Eichler H.-G. , 2017) discusses the current situation and the next steps: there is an urgent need to demonstrate the validity of non-randomised data, and create a common infrastructure for generating this data in Europe in order to calm the retractors of MAPPs who criticize that the EMA is lowering the evidence state and that the validity of RCTs is being undermined by RWE.

As already pointed out above, it also needs to be clear to all stakeholders that MAPPs do not aim to deviate from the current legislation which requires the benefits to outweigh the risks to grant CMA or MA (European Commission, 2001, S. Art 1 (28a)). Furthermore, efficacy will not be left out of the equation as is being insinuated by other regulators such as FDA. CMAs are one of the useful tools to serve the purpose of MAPPs from the regulatory point of view; but, the concept of 
"conditional" needs to be further discussed with downstream stakeholders in order to avoid the current refusal it generates. The wrong impression that "conditional" equals not having any evidence on efficacy or safety needs to be corrected and all stakeholders need to understand the basis for granting CMAs and agree on their validity.

Finally, MAPPs want to solve the conundrum of patients wanting to have access to drugs as early as possible and decision makers wanting to have as much information as possible on the safety, efficacy and effectiveness of the drug before it reaches the market. It also looks at reducing the inherent uncertainty or earlier approval to the minimum as fast as possible. With adapt-smart, the focus has shifted from claiming the need for new regulatory pathways to developing new evidence generation tools in order to have the right tool-box for each potential drug candidate.

\section{Pillars of APs: Conditional marketing authorizations}

As presented in chapter 4, CMAs have not been widely used since its inception in 2006. However, as concluded, they constitute a good tool for flexible marketing authorization and should be planned prospectively as the regulatory pathway to follow in drug development. The APs report (European Medicines Agency, July 2016) elucidates that some companies have already voiced their wishes to follow the conditional approval regulatory route and hopefully, the APs project and ADAPT SMART have raised awareness about the availability of flexible approaches such as CMAs.

The EMA published a 10 year report in January 2017 on the CMAs released between 2006 and 2016. The report also presents data on the post-authorization commitments and conditions set in CMAs and whether they have been carried out and controlled. It also evaluates if the MA was finally given, taken away or new conditions were set. According to the report, 30 conditional approvals were provided between 2006 and 2016, of which more than half were in oncology. From the 107 post-authorisation obligations, 75\% related to final results from clinical trials, most of them were delivered on time, and none presented negative results that turned the risk-benefit ratio negative ${ }^{21}$. Furthermore, on average it took a medicine 4 years to receive full MA after CMA (EMA, January 2017). These conclusions are promising, specially regarding the aspect of timely delivery of answers to the conditions. However, the total number still remains very small and accounts for an average of 3 approvals per year since 2006.

The discrepancy between the number of CMAs evaluated in chapter 4 (17) and the number of approvals discussed in the EMA report (30) is due to the fact that the

${ }^{21}$ Only in the case of Translarna (indicated for a small subset of Duchenne Muscular Dystrophy patients), the results of the post-authorization requests remained inconclusive and the company was requested to produce more evidence to support the benefit-risk ratio. 
EMA report included CMAs granted in 2016, CMAs of medicines withdrawn from the market and medicines which later received full marketing authorization. All these drugs were not included from the analysis presented in chapter 4 due to the time of publication (2015) and a limitation on the methods to search for granted CMAs, which only included current approval status and not drugs that were initially approved with conditional approval. Nevertheless, the main aim of the article was to evaluate if the criticism relating to the need of implementing new regulatory instruments for early patient access to personalised medicine was founded. The conclusion of chapter 4 "appropriate regulatory tools already exist and should be better used" is still valid, very relevant and begins to be accepted by the drug development community. In addition, Chapter 4 questions the validity of the conclusion in chapters 2 and 3 regarding the need for new regulatory tools and rejects it. Therefore, the reader should consider chapters 2 and 3 superseded by chapter 4 on the topic of developments of new regulatory methods.

The analysis presented in the 10 year report published by the EMA (EMA, January 2017) should be repeated regularly if CMAs were to be applied more often. It is very important that an embedded control system is in place to ensure that all conditions are met. Furthermore, other mechanisms could be implemented to reduce uncertainty:

- the re-evaluation of the risk-benefit ratio after a certain number of patients have been treated with the product to see if many safety signals were missed due to the low number of patients treated in clinical trials and the earlier approval;

- increased follow-ups with developers regarding the status of the conditions set in the approval and safety data. This is already done yearly through the PSUR (periodic safety update report) for all drugs with MA, and in the revision of the CMA, which is valid for 1 year, but could be considered to be done more often for conditionally approved drugs.

These control steps should be embedded in any flexible marketing authorization method with set timelines and methodologies (see below Figure 8-4).

\section{Pillars of APS: Systematic early dialogue}

In addition to CMAs, another essential component of APs and MAPPS is the implementation of a systematic iterative dialogue process, which involves all relevant stakeholders early in the drug development process.

Systematic early dialogue is an essential stone of any flexible regulatory method. It reduces the uncertainty for all stakeholders, reduces the waste in data generation that is not relevant for decision makers and helps to plan resources ahead for future applications. As pointed out in ADAPT SMART, the safe harbour (non- 
binding) nature of the discussions also facilitates an open dialogue that is not guaranteed during the scientific advice procedure.

The newly established program on PRIority MEdicines (PRIME) by the EMA looks to promote systematic early dialogue for medicines that address an unmet medical need. Specially focusing on SMEs (small and medium enterprises) and facilitating their access to the safe harbour discussions very early in the development process. This is an important step in order to avoid the loss of promising medicines because of a badly designed development (EMA Guideline, 2016).

One important aspect is how to find the right time to start the dialogue, this time point must be early enough in development to promote useful discussions between decision makers and developers; but late enough so that data from the necessary safety and proof of concept trials is available to know if the compound is promising and development should continue. In order to find the appropriate point in the development timeline, future research should focus on analysing the development programs run up to this date and see if there is a common inflexion point in the decision to continue or not with the development of drug candidates. This point can be called "exit phase" (see Figure 8-1). Once a promising drug has successfully passed through the "exist phase" the developer can send the application to the coordinating body (e.g. EMA) to start the early dialogue procedure.

Future research should concentrate in defining a systematic process for early dialogue to create truly systematic early dialogue. Important aspects to be defined are the time points and the stakeholders that need to be in contact at each decision point.

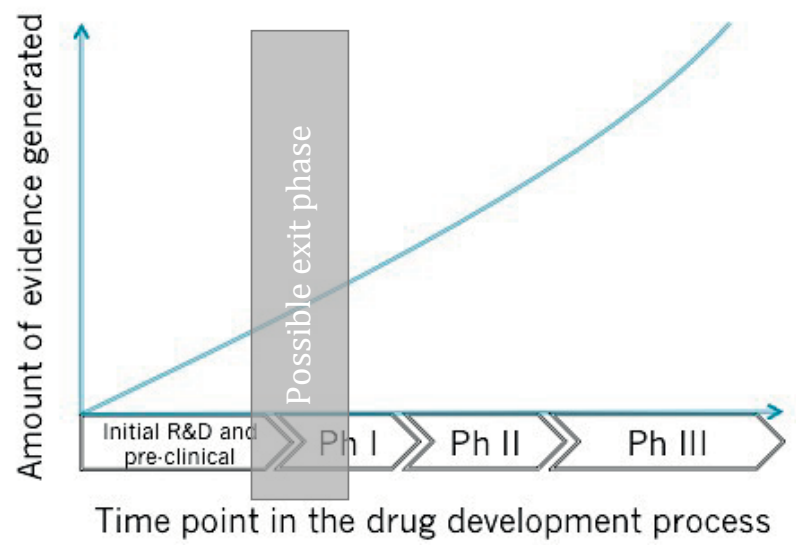

Figure 8-1. Exit phase in the drug development process. After successfully passing through the checkpoint, developers request the early dialogue procedure. Modified from oral communication with Dr. Nikolaos Evangelatos. 


\section{Pillars of APs: Real World Evidence and big data for the benefit of the regulators}

The third pillar of ADAPT SMART and APs is the use of evidence that was not produced in an RCT for decision making. It is also one of the most discussed points, since many stakeholders do not accept the validity of evidence from observational trials, registries or other non-randomized non-interventional studies (see Figure 8-2). The definition of RWD and RWE is well described in the IMI GetReal report, which offers the view from different organisations globally and all the definitions published until now (GetReal, 2016).

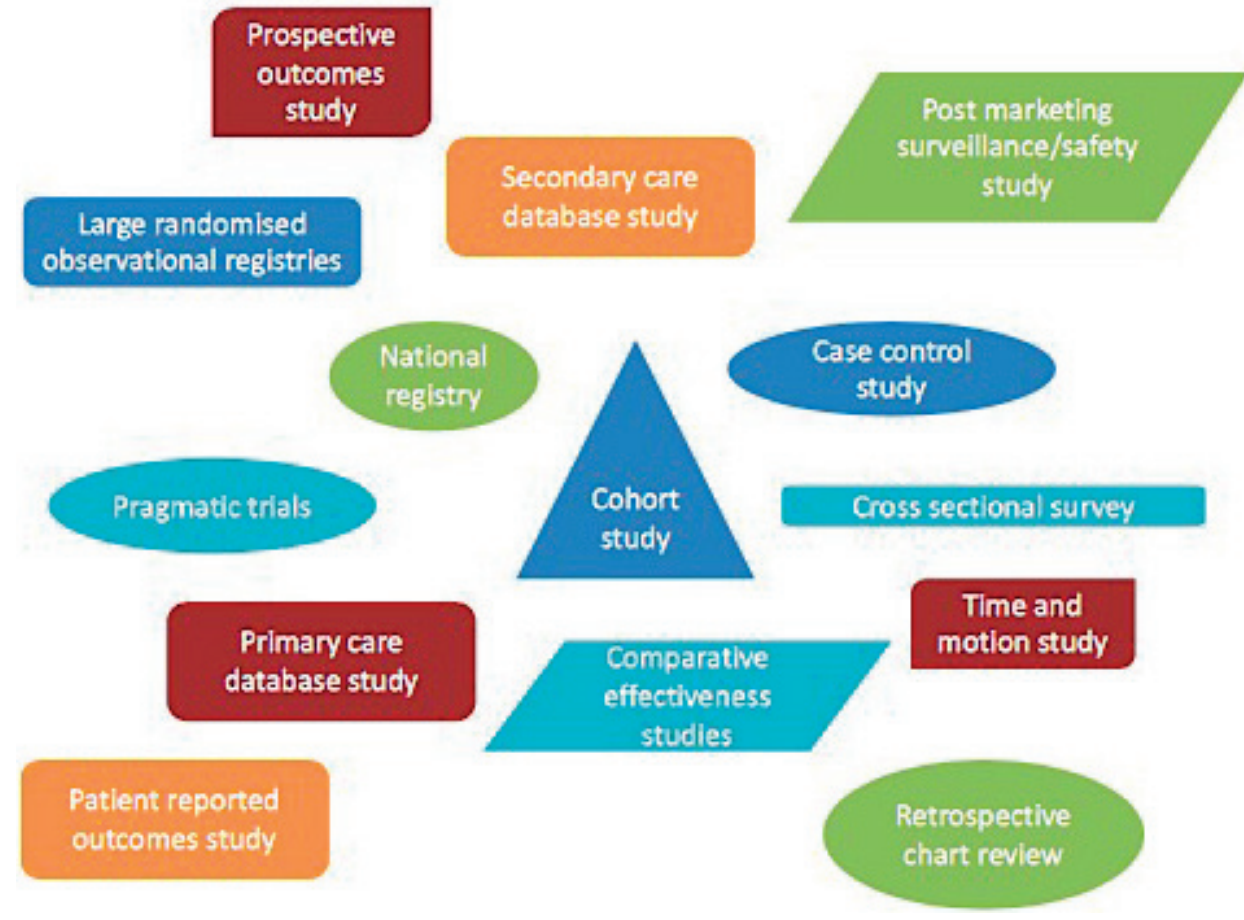

Figure 8-2. Examples of real world studies. Real World Data can be collected from various sources, as the studies depicted above. The analysis of this RWD converts the data in evidence to be used for different purposes. From (ABPI, 2011), (GetReal, 2016).

An important aspect to consider is that real world data (RWD) -and real world evidence (RWE) produced therewith- puts into the evidence equation the effect of the drug in routine clinical practice in the wider patient population, whereas RCTs describe the use of the medicine in an ideal World. Very often patients suffer comorbidities and take several medications concomitantly; these "normal" patients are excluded from usual clinical trials. RWE and RWD apply better to the normal patient population that doctors are faced with in their practices. Moreover, some of the questions asked by the regulators, HTAs and payers to establish the risk- 
benefit ratio, and more importantly the relative effectiveness, can only be answered by RWE (Sherman, et al., 2016).

Some of the big challenges with RWE / RWD is how to ensure the quality of the data, how to avoid bias and how to make data recollection systematical. These aspects are being addressed by the European IMI project Get Real; which is focusing exclusively on RWE / RWD and showing how it can be implemented in the drug $R \& D$ process and healthcare decision making. In order to tackle the critics of RWD, it is paramount to demonstrate the scientific validity of the data and how the quality standards can be kept high.

Big data and the tools discussed in Chapter 6 can be of use in this domain; especially to analyse the amount of data produced in non-randomized noninterventional studies. However, the necessary IT infrastructure needs to be set up in Europe in order to standardize this controlled observational data collection to use it as a decision supporting tool for regulators and HTA bodies. The ideal scenario would include an automatized system across Europe that allows the automatic recollection of RWD in medicines with CMAs, and eventually for all medicines. This can only become a reality when electronic health patient records (EHRs) are implemented in all healthcare service providers and the information is fed into well-designed registries. There are two projects that should be followed closely in this aspect: the healthdata.be initiative set up by the Belgium government which is creating the infrastructure for registries and linking them to the EHRs; sponsors are then obliged to set a registry for drugs under outcomebased MEAs that use the public infrastructure, are owned by the company and must be made available for public use (ISP, 2017). In addition, the Big Data for Better Outcomes IMI project focuses on disease specific registries and data sets, on how to use them, how to improve them and how to extend good practices to other fields.

Even though there is much criticism to the whole project and the concept of APs, the implementation of the separate components from MAPPs can already be perceived in the drug development landscape: indications target smaller patient populations with high unmet need, non-RCT information is starting to be accepted in the decision making process and outcome-based managed entry agreements with flexible conditions are being developed (CRA, November 2016). In a project where it seems that the sum of its parts is greater than the whole, it is not important if the sceptics criticize the whole but embrace the application of the parts. 


\section{Downstream stakeholders: the need to consider the new approaches}

As discussed in chapter 5, early access is not possible if the so-called "downstream" stakeholders (HTAs, payers, etc.) do not accept CMAs and are not willing to consider therapies that have been approved under flexible regulatory approaches. The view of the different bodies in Europe varies, NICE is involved in many of the IMI projects and takes a sceptic but open view, whereas the German counterpart IQWIQ is completely against it.

The EMA has been the driving force in implementing the APs pilot in Europe, we may ask ourselves whether this advance could have been possible if the European Agency did not exist and the 28 competent authorities had to promote the change. In this line, the section below discusses how a Europe-wide HTA body may move forward the discussions at their level and finally allow timely patient access accepting higher uncertainty for certain promising drugs until more confirmatory evidence is available.

\section{The EHTAA: an EU-wide HTA body}

As proposed in the ENVI report (European Parliament, 2015), European Member States should join their efforts in performing the evaluation of relative effectiveness or added therapeutic value through a joint coordinating body. In this work, the body will be designated as either European Health Technology Assessment Agency "EHTAA"22 or European Medicines Relative Effectiveness Agency "EMREA". This agency would coordinate the work of all health technology assessment agencies in Europe. As in the centralised drug approval procedure, member states could share the task of evaluating relative effectiveness to more effectively use their internal resources. A rapporteur agency and a co-rapporteur agency would perform the evaluation, with the input of all other agencies, which wish to comment, and then all national (or regional) HTA agree on the conclusion of the evaluation. In this model, and as proposed by the ENVI report, the result of the evaluation can be implemented in each healthcare system depending on the national or regional policies on P\&R.

There have been various projects to initiate the collaboration on HTA in Europe. The Joint Action (JA) EUnetHTA was established in 2010, it is now in its third funding round that is ensured until 2020. Many have argued that this JA could overtake the role of the European coordinating body. So far the work has been very good to start a common dialogue and the European cooperation. However, the joint reports are very rarely implemented nationally and they lack the legal basis

22 "EHTAA" is better suited to implicitly include other health technologies that could benefit from the process. 
for their enforcement. An additional HTA Network was established in 2013, with the role to coordinate the national HTA agencies focusing on strategy and policy aspects; even though participation is voluntary, all European HTABs are represented showing their high interest in collaboration (European Commission, September 2016).

Various reports have been recently published In Europe regarding a coordinating HTA agency. In addition to the ENVI report, the European Commission has published an inception impact assessment (European Commission, September 2016) showing how important this topic is for the European stakeholders. The inception impact assessment describes the current situation and presents 5 possible future scenarios: from these scenarios, option 4 seems the most adequate one to really work together and get one common relative effectiveness assessment (REA) in Europe which is implemented in the MSs (to avoid duplication of efforts), but each region retains the competence to decide how the REA is translated into their P\&R decisions.

Criticism against a joint evaluation body is expected. Many member states fear losing their competences and do not see the added value of joining efforts and resources. 20 years ago the EMA was not possible in the mind of the EU and now it celebrates its 22nd birthday having harmonized the MAA process in Europe without taking the main competencies away from the Member States and coordinating their efforts. This is essential to make Europe stronger and more competent to compete on equal terms with USA and other regions. Now the EMA has become one the most important regulatory bodies globally, and the one many regions look up to in order to replicate their work and guidelines. Many can argue that HTA is different to MAA in that there are many national aspects to be taken into account. However, the comparative effectiveness aspect of HTA can be harmonized in Europe and applied everywhere from a scientific point of view; challenges like different comparators in different regions can be solved in the methodology. Nevertheless, politically it is a more sensitive matter that will need long discussions and negotiations until a solution is found (Eichler H.-G. , 2017).

For our purpose, a centralised coordinating body would facilitate the discussion on flexible HTA models discussed in chapter 5. As the EMA takes the leading role in the discussions to develop flexible regulatory approaches for MAA, the EHTAA (or EMREA) could do the same for the P\&R process and its steps.

\section{Flexible pricing: the need to find better models}

The last step before patient access is the negotiations and decisions on P\&R, which often use the findings from HTA. Within the ADAPT SMART project a report has been published on the use of Managed Entry agreements (MEAs) for products with CMAs (including products approved under exceptional circumstances) (CRA, 
November 2016). As in the report from Ferrairo and Kanavos, they conclude that MEAs are not widely used, especially for products with CMAs, and that they are only applied in a few countries (Sweden, Italy, Poland and Czech Republic) and mostly relate to financial agreements (such as volume based agreements and discount schemes). Payers favour simple MEAs since more complex outcomesbased agreements are resource and time intensive. The automatization of data collection in registries, through EHRs or other ICT would make the wider application of MEAs possible. Furthermore, one of the conclusions of the report is that, the regulators and HTAs/payers need to come to an agreement on the evidence required for the decision making process so that part of the evidence can be used simultaneously to respond to the conditions set by the regulator and to the requirements of the downstream decision makers.

In addition to the wider implementation of MEAs, different pricing mechanisms are being proposed and implemented, such as value based pricing in the UK. Going one step further, entirely new drug development concepts involving increased public investment and public benefits are also discussed (KCE, June 2016). What is clear is that the increased market entry of more targeted drugs, which can only be applied in a small patient population and therefore have very high prices, challenges the sustainability of our health systems. We need to find alternative P\&R mechanisms that include flexible pricing including risk-sharing. In a future with more CMAs it is reasonable to not apply the full price before the uncertainties regarding risk and benefit are reduced; one proposal would be that the marketentry price is low and the price increases according to the evidence provided so that it reaches its full potential in the mature phase of marketing. Whatever mechanism is implemented, it must not be resource intensive for the stakeholders involved in order to ensure its uptake. Nevertheless, we cannot be short-sighted in healthcare: the long-term financial and societal profits of outcomes-based MEAs and other flexible pricing mechanisms should be weighed against the immediate short-term resources needed.

Following the idea of the EHTAA, a European wide procurement agency for medicines could be created (Brand \& Brand, 2017). The added value of the agency would be its negotiation power: it negotiates for more than 500 million Europeans, whereas each country negotiates for a smaller population (maximum of 80 million). The agency could then address the paying capabilities of the different regions in Europe by assigning differential prices according to the country and managing the flow of payments internally. Such a procurement agency could also pilot the implementation of Europe-wide transparent outcome-based MEAs and manage the development of the corresponding automatized ICT infrastructure (analogue to healthdata.be). Even though it would benefit many countries, it would lower the prices and it would ensure market access for all European patients, it is a very difficult topic politically. 
We should turn to the BeNeLuxA initiative and learn from their pioneering project in cross-country collaboration: Belgium, Netherlands, Luxemburg and Austria have started a pilot in 2015-2016 that plans to conduct joint HTA, horizon scanning, information sharing and joint pricing and reimbursement negotiations (KCE, 20 April 2017). With the formation of technical task forces and product specific coalitions these countries will join forces in HTA and drug procurement and avoid duplicities of work. Their lessons-learned could be applied to create the EHTAA (or EMREA) and the European joint procurement agency for health technologies.

\section{Applications for personalised medicine}

The previous sections present how relevant the topic of flexible regulatory methods and timely access to medicines is in Europe and globally. For personalised medicine, the use of flexible pathways and the increase in conditional approvals is essential. On the one hand because personalised therapies are often created for areas of high unmet need and can be applied inherently only to smaller sub-set of patients, fitting into the conditions for adaptive pathways. On the other hand, because targeted medicines have the potential to be very effective in a small patient population, and would benefit of timely market entry (once the positive risk/benefit ratio has been established with limited evidence), controlled prescribing and further evidence generation on the market to support a full marketing licence. Controlled patient access (see Figure 8-3), or controlled prescribing, in the initial market entry phase of drugs approved under CMAs is an essential point for personalised medicines. Severe adverse reactions can be avoided if prescription is better controlled in the initial marketing phases.

Since many medicines are being developed in the direction or targeted and personalised therapies, we should no longer speak of a special regulatory landscape for personalised medicines, but from the general regulatory landscape. This is why the previous and the next sections have not focused exclusively on the regulatory framework for personalised medicines. All proposed actions and defined projects are for the general regulatory framework, with the idea that most medicines will become personalised or targeted in the future and the general framework must be applicable to all of them. 


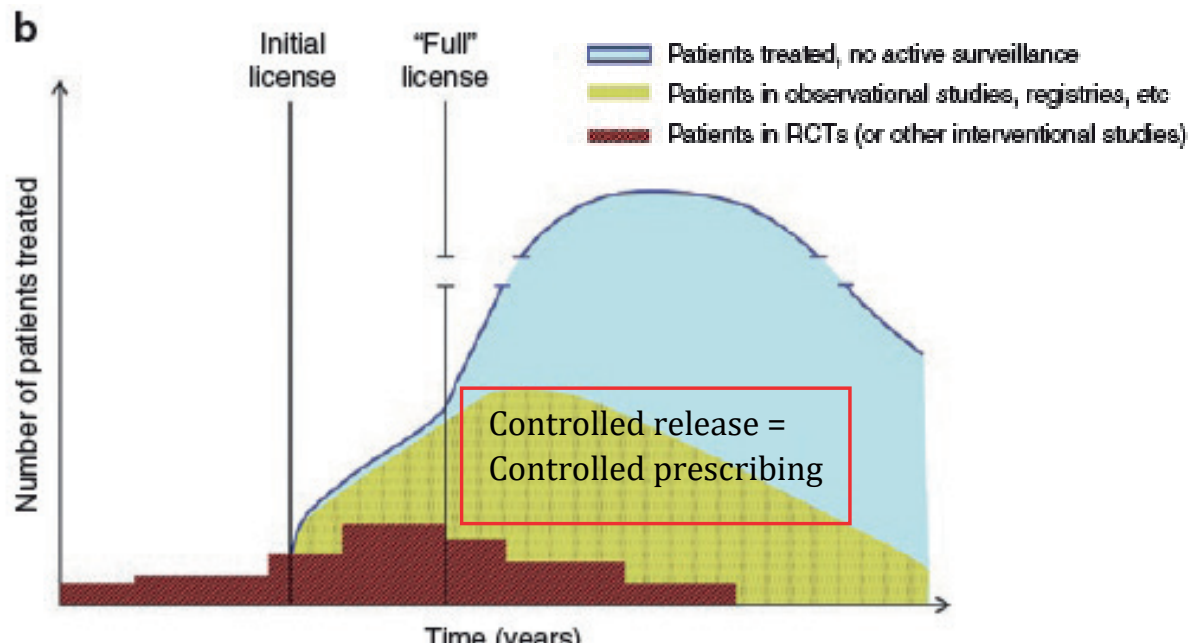

Figure 8-3. Proposal for adaptive pathways with more data being collected in the controlled market entry phase after conditional approval. It is essential that there is a review of the evidence produced in this controlled phase in order to provide the full license. Modified from (Eichler, Oye, Baird, \& et al., 2012)

\section{Proposed framework for regulatory evaluation and approvals: using existing tools}

Many publications claim a complete change in regulation for the implementation of personalised medicines and for the drug development process in general (see chapters 2 and 3); however, we cannot expect a disruptive change in the current regulatory environment for drug development, marketing authorization or market access. It is more feasible to implement a progressive change using the available tools and integrating new tools that adapt the system to the current technological advances and knowledge base, making it more efficient for all stakeholders.

Chapter 4 describes the tools for flexible MAA and concludes that the tools for the changes claimed as necessary by many reports already exist in Europe but are under-utilised. Building on figure 7 from chapter 5, where all the new instruments in Europe and the USA are depicted, to propose a framework for drug development that uses existing processes to accelerate patient access to promising drugs, including personalised therapeutics (see Figure 8-4). This framework is already being applied implicitly but has not been described explicitly and is not systematically used. 


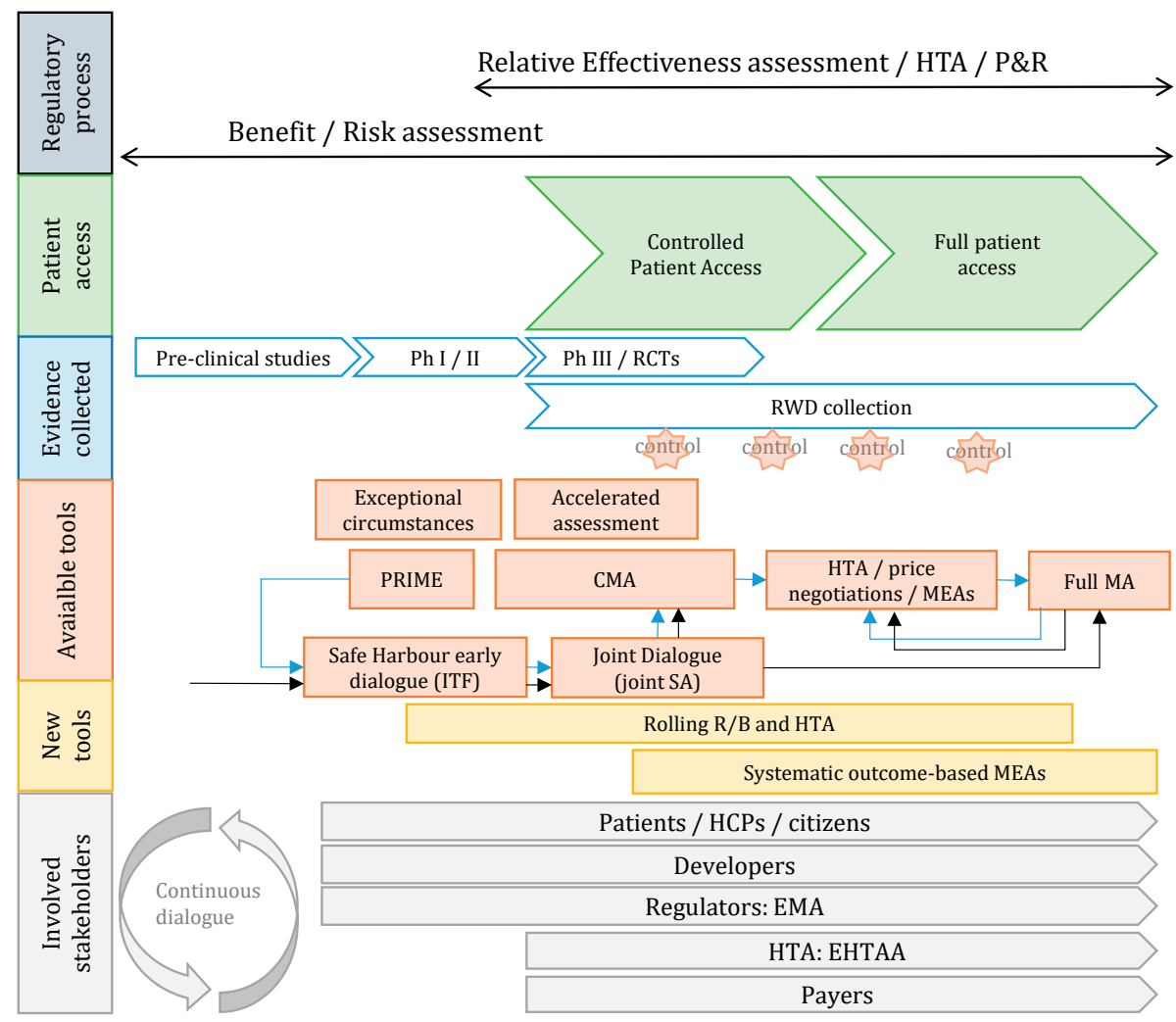

Figure 8-4. Framework of regulatory tools for timely patient access to innovative medicines. See text below for a description. CMA: conditional marketing authorization; EHTAA: European Health technology assessment agency; EMA: European medicines agency; HCPs: health care professionals; HTA: health technology assessment; ITF: Innovation task force; MA: Marketing Authorization; MEAs: managed entry agreements; P\&R: pricing and reimbursement; Ph I / II / III: clinical trial phases; PRIME: priority medicines scheme; R/B: risk / benefit; RCT: randomised clinical trials; RWD: real world data; SA: Scientific Advice. The order and position of the tools and stakeholders does not correspond to the time according to the R\&D timeline presented under "evidence collected". The blue files mark the pathway for PRIME designated drugs; the black file depict the pathway for other drugs, which can go under CMA (and then join the blue path) or under MAA.

The first three boxes on the left hand side of Figure 8-4 represent the regulatory process, type of patient access and evidence collected. The top presents how the regulatory process starts very early in the development process, accounting for the early multi-stakeholder dialogue, including the involvement of stakeholders from HTA and P\&R very early on. After the initial phases of clinical trials, if the evidence shows a positive B/R ratio, CMA can be granted facilitating early patient access to the drug in a controlled prescribing phase in a reduced patient population. After more evidence is collected, the developer can present the answers to conditions to receive full MA leading to full patient access. The framework also includes the early 
collection of RWD in parallel to phase III clinical trials, which is essential to gather evidence in the CMA pathway.

The available and new tools presented thereafter have already been described in other sections and will not be described in detail here. Iterative early dialogue and multi-stakeholder dialogue are essential stones of the framework. The needs of developers (applicants), regulatory bodies, HTABs and payers must be aligned very early in the development process to plan it accordingly and make it more efficient. Developers can access safe harbour discussions, through the Innovation Task Force (ITF) at EMA, or through PRIME if they qualify for the scheme (specially targeting small and medium enterprises (SMEs)). Thereafter, the more structured advice process of Joint Scientific Advice at the EMA can be requested. Our framework includes payers very early into the iterative dialogue, who do not usually participate. EMA, EHTAA and a European Payer representative (e.g. MEDEV or ESIP) can coordinate these advices and represent the view of all the national bodies.

After the SA rounds, applicants can submit their applications through either of the routes they had planned (CMA or full MAA). With CMA, patients can access the drugs under controlled prescribing until additional evidence is collected, revised and the CMA is converted into full MA, if it is positive. Exceptional circumstances and accelerated assessment are further tools that facilitate earlier patient access under certain conditions (described in chapter 4).

After CMA or MA are granted, the HTA, price and MEA negotiations start. The early multi-stakeholder dialogue round should have prepared the ground for the necessary evidence for P\&R process to be collected early in development and to be readily available at this stage. For CMAs, once the full MA is granted, a new $\mathrm{HTA} /$ price/MEA round of negotiations can take place to establish the final market access conditions based on the new available evidence, which may change the value of the medicine.

The new tools of rolling R/B assessment and rolling HTA assessment where proposed in chapter 4 and are already in use at the FDA. It is in fact a continuation of the iterative dialogue rounds and facilitates the final evaluation at CMA and MAA because the data is already familiar to the regulator. However, this system is resource intensive for all stakeholders and its benefits have to be weighed against its weaknesses. Systematic outcome-based MEAs are proposed in chapter 8, this risk-sharing approach between manufacturers and payers would bring an enormous added value to the healthcare system; however there are some pitfalls such as availability of data and need of intensive resources that are discussed elsewhere in this work.

At the bottom of the image, the stakeholders are presented. HTA and payers can be involved later than regulators and patients; however, they still need to be involved 
early enough to consider their needs for evidence generation. To support the process or $\mathrm{P} \& \mathrm{R}$, the EMA is considering how to improve the European public assessment report (EPAR) to include a component of clinical value and facilitate the evaluations at the HTA and payers (EMA and EUnetHTA, 2016) (Eichler, Hurts, Broich, \& Rasi, 2016).

Finally, flexible authorization methods are welcomed but need to have appropriate risk minimization measures to reduce the uncertainty for patients that receive the therapy on the market. Recurrent control steps until the final authorization is provided to see if the evidence has shifted the R/B balance in one or other direction (depicted in Figure 8-4), careful and controlled prescription to patients, stress tests for the available monitoring mechanisms, and a critical evaluation of the entire flexible MAA procedure after a certain number of procedures have been performed could help to minimise the risks, make a resilient system and create the best regulatory approach.

\section{Challenges}

This section discusses the challenges of implementing flexible MAA and pricing approaches, the challenges faced by the regulators as well as the challenge of limited literacy. The challenges regarding the development and implementation of personalised medicines have been thoroughly discussed in chapters 2 and 3 and will not be discussed here.

\section{Flexible regulatory approaches}

Even if the tools for timely patient access are already available, we cannot ignore that scaling up their use poses many challenges for all stakeholders.

The first challenge is for developers to decide what drug candidates to consider for CMA and when in the development process it should be considered. Even though the scope given in the CMA guideline (EMA Guideline, 2016) and the PRIME guideline (EMA Guideline, 2016) can help with the decision, CMA has been mostly used as last resort at CHMP and not planned by the company in advance. More prospective planning of CMA is needed so that the evidence, the timelines and the target population can be tailored to the requirements for CMA, which differ from the MAA. Importantly, early access and CMA may not always be the best solution for public health: there is no use in bringing it earlier to the market if the access will be limited and less patients will profit from it than with a normal MAA, as presented in the example in chapter 7.

The additional rounds of scientific advice and safe harbour dialogue may bring organizational challenges for all parties involved including the developers. Most 
companies do not have the right structure to discuss market access plans early in development, or to have cross-department involvement and dialogue. However, this can be easily solved as the companies gain more experience with the process and adapt their structure or create cross-functional teams. In addition, these tools are very useful but resource intensive for the decision makers as well as the developers, new positions need to be created for people to coordinate the dialogue and the experts who give input need to re-allocate some of their existing tasks.

There are also challenges in the post-CMA phase. The post-authorization measures (PAMs) need to be well planned and feasible. It should be considered whether it is ethical to perform an RCT with placebo once the product is on the market under a CMA. Or it might not even be feasible to recruit patients into a clinical trial, because they can access the drug on the market. The only solution for these cases is to perform the study in countries where the drug is not yet available.

In addition to RCTs, other data sources may be more appropriate to answer PAMs. All stakeholders need to be aware that RWD are better designed to answer some questions that cannot be answered by clinical trials. Ensuring the quality of RWD is very challenging, but necessary if we want to use it as evidence for decision making and convince the reticent stakeholders that RWD and RWE are useful sources of information.

Once the drug is on the market under a conditional authorization, regulators are faced with the dilemma of the PAM results: what to do with the consequences of negative results from PAMs that tilt the $B / R$ balance to negative, or with results that are delivered with very large delays? In such cases, the CMA should be revoked. However, the strength of the "perceptive" value of the drug for patients and carers cannot be undermined. Even if the medicine does not have a positive $\mathrm{B} / \mathrm{R}$ ratio, patients and carers may value it very highly because it offers a small improvement or simply an opportunity to treat the disease and it gives hope. Since 2006, the EMA was not confronted with this situation since they did not receive negative PAM results in any of the 30 CMA procedures, or significant delays in the delivery of results (EMA, January 2017). Only in the case of Translarna, approved conditionally for a subset of DMD patients, the results received were inconclusive and the developer was requested to produce more evidence (Salmonson, 2017).

On flexible pricing approaches two of the most important challenges are the fact that outcome-based MEAs are very resource intensive for the payer and the lack of infrastructure in place for the continuous monitoring of the medicines' use on the market. Finally, as described in chapter 5, the miss-alignment with HTAs and payers needs to be solved: HTAs and payers have to understand that the benefit risk balance is positive in CMAs, but the evidence package used to decide on the $\mathrm{B} / \mathrm{R}$ is smaller bringing more uncertainty to the equation. 


\section{Regulators}

Every change brings challenges for all parties involved, including decision makers. In this area, the new technologies in drug development (pre-clinical models, computer simulations, etc.) and the more complex therapies (nanomedicines, organ printing, personalised medicines, faecal transplants) also pose challenges to the regulators who have to evaluate these methods and products. In most of these areas there have been reviews conducted to analyse the current regulatory landscape that claim a change in regulation is needed (chapter 1, and (Sanhai, Sakamoto, Canady, \& Ferrari, 2008)). However, we should look at the available tools and how to apply them or slightly modify them to fit the new technology, instead of requesting a radical change. Furthermore, there may be situations when it is not possible to cover all the requirements of a new product class with one guideline, as in the case of nano-technology based products, which is why regulators have to resort to the case-by-case approach (FDA, 2014). As it is stressed in many guidelines, developers and applicants can deviate from guidelines and regulations if it is based on scientifically sound arguments, the regulator will consider the evidence provided and the arguments for the deviation. Developers complain that this lack of specific regulation brings uncertainty to the regulatory process; however, they also need to consider that if all the scenarios cannot be built in a guideline and each application needs an exception, the guideline does not provide more certainty.

The exponential increase in data and information (as seen in chapter 6) is also a challenge for regulatory agencies. Artificial intelligence could be used in order to solve this problem as described below. Furthermore, the activity of horizon scanning, can help to become more proactive and move from the current reactive approach.

\section{Literacy}

Another one of the biggest challenges the system faces is the lack of physicians' and patients' literacy. It has become evident to the author that many of the stakeholders directly involved in the drug development process and the drug's journey from the lab to the patient do not understand the implications of MAAs, CMAs, HTAs and P\&R for effective patient access. In order to improve the exchange and the understanding between stakeholders it is essential to create a common language and common knowledge base. Promising drug candidates that are developed in academia might never reach the patient because of lack of regulatory knowledge from the academics involved. Therefore, an update to the curricula of physicians and other related scientific disciplines is paramount for this goal.

Students in medicine, biology, biochemistry, bioinformatics, biomedicine, pharmacology and all their branches should receive at least 2 new modules: one 
related to regulatory sciences and one related to upcoming new technologies where data sciences, personalised medicine and other important updates should be taught. With a rapid change in this field, the old curricula are becoming outdated; the professionals of the future need to be familiar with the new technologies (e.g. use of big data) they may need to use in every day practice, and the new riskmodels for drug approvals as well as their implication in drug prescription and use (e.g. Adaptive Pathways).

\section{Other challenges}

As we learn more about the biology of diseases, we understand that dynamic biomarkers are very important. Tumours evolve generating spatial and temporal tumour heterogeneity with different cell types and different biomarkers (Fisher, Pusztai, \& Swanton, 2013). This is very relevant for personalised medicines with companion diagnostics, for biomarkers used as prognostic factors, for the drug development process if biomarkers are used as surrogate endpoints for decision making, and for clinical decision making since more than 1 drug may be necessary in cases of intra-tumour heterogeneity. Moreover, clinical trials often recruit patients based on historic biopsies, and the results of biomarkers from previous tumours are being used to treat new lesions or metastases. Thus, it is very important to keep in mind that static biomarkers may not be valid at the point of decision making, be it regulatory or medical.

Finally, there is an organizational and behavioural challenge: the most difficult part of this thesis was to access the relevant information and find all the projects involved in personalised medicine, flexible regulatory approaches and timely patient access in Europe. There are many interesting European projects that happen in silos with limited public information and not easily accessible for interested parties. Furthermore, the dissemination exercise of most of them is not well managed which means that once they are finished it has very little impact in the field. All stakeholders should be aware of all the projects that affect their work and the possibilities they have in drug development. A comprehensive mapping exercise covering all stages of drug development and regulatory processes could be performed in order to give an indication to drug developers and other interested parties on what is happening.

We should not hide in our small projects protecting them from external parties. Patients and society as a whole can only benefit from our efforts as scientific and regulatory community if we join forces and collaborate, to find the best projects, push them forward, and inform the downstream interested parties about the progresses made and the findings: this is the only way to have an impact on public health. 


\section{Forward look}

If we take one step away and analyse what needs to be accomplished in the near future, we should incentivise that new innovative medicines are developed to address the needs of patients and society. Companies often give more consideration to their ROI than to the needs of patients and society when taking decisions to invest in R\&D, which is not surprising and normal practice for the private sector. This is why they often need external incentives to develop what patients need. There have been some initiatives in this regard in Europe with the orphan drug regulation (EMA, 2007) which has incentivised the development of therapies for rare disorders, furthermore, the European patient group on rare diseases EURORDIS has also managed to steer innovation in the direction of rare diseases with their input and lobbying. At the EMA, the PRIME scheme addresses this problem by giving priority medicines a designation for indications of high unmet medical need. And some countries have tried to develop incentive mechanisms for areas of unmet medical need -as presented below. However, how is unmet medical need defined and measured? A quantification method for unmet medical need could be developed to avoid a subjective judgement and standardise its application. Firstly all stakeholders need to agree on the definition. In addition, the use of flexible regulatory approaches should be expanded to all conditions with unmet medical need, and not restrict them to life-threatening or seriously debilitating diseases as it is now in the regulation; there might be conditions with unmet medical need that do not fit this definition (Salmonson, 2017).

In the decision making process in drug development, as discussed in chapter 5, HTAs need to open the dialogue and welcome discussions on flexible approaches. The first step would be to join forces towards the standardisation of RWD recording and improving all the pitfalls instead of criticizing its use. To ensure the Quality of RWD, the international community could create a set of guidelines similar to the GXP guidelines for "good data recording practices", which include automatized remote monitoring and data validation. Furthermore, we could look at other medicine classes, like ATMPs, where after the MAA companies are obliged to set up a registry (EMA, 2008). This could be applied to all drugs and eventually to all diseases. If the registries are complemented with the establishment of "health data cooperatives" or groups of patients that collect and own their data (Hafen, Kossmann, \& Brand, 2014), the use of RWD and RWE could increase exponentially. Moreover, these registries and health data cooperatives could be standardised to increase their data quality and their potential use.

The sentinel program in USA already uses RWD for pharmacovigilance purposes. After seeing the success of this program, the Senate and the Congress drafted the $21^{\text {st }}$ century cures act that was signed by President Obama into law in 2016. This Act obliges the FDA to put up a system that uses RWE for efficacy as well as for 
safety, and have it in place by 2018 (Califf, 2016). With this short timeline, Europe needs to act quickly to avoid staying behind in the use of RWE in drug development and regulatory decision making.

Regarding multistakeholder dialogue, payers should also be included into parallel advice procedures. Since they are part of the last process in P\&R and may have specific requirements that need to be planned early on. The Medicines Evaluation Committee (MEDEV) and the European Social Insurance Platform (ESIP) represent the payers of $14 \mathrm{MSs}$, they could be taken as the main partner in early dialogue to sit at the table with EMA and HTAs in parallel scientific advices (EURORDIS, EPF, 2015). Furthermore, the process established by the voluntary scheme set up for rare diseases (Mechanism of Coordinated Access to Orphan Medicinal Products, MOCA) with representatives from developers, payers, HTAs and patients (MoCAOMP, 2013) could be expanded to other disease areas and integrated into the current multistakeholder and early dialogue initiatives at the EMA so that the entire drug life cycle is covered. Moreover, companies need to change their structures to potentiate the functioning of multistakeholder dialogue. As A-V Eggiman from Bluebird Bio put it: "we need to create adaptive company organizations which rethink how companies are organized in silos, create adaptive clusters and seek multi-stakeholder input on evidence generation through the lifecycle of the product" (Eggiman, 2017).

Continuing the work in chapter 7, which describes a niche use for game theory for the first time, future work should further exploit the use of game theory in drug development. Furthermore, it should also analyse tools used in other industries. Technology assessments are also widely applied in other public areas (e.g. infrastructure and road construction) with different approaches than in health. A combination of these approaches could help create HTA 5.0 and even automatize the process. This automatisation would also exploit the full potential of HTA and extend its use to all the components of healthcare beyond medicines and medical devices.

As already discussed above, artificial intelligence is also a field that has not been exploited in health care and that could bring great advances. The application of Watson and other decision making programs that help physicians make clinical decisions is a starting point to enter the healthcare market; however, its use could go beyond and help in all steps of drug development and healthcare including the regulatory decision making step. Artificial Intelligence could help regulatory bodies to analyse the increasing body of evidence available and point out to hidden signals that may not be obvious and are not included in the submission package companies send for the MAA. As with the clinical decision supporting tools available for physicians, artificial intelligence is not an instrument to replace the experts in regulatory bodies, but a facilitator of the review process that digests the enormous amount of evidence available and presents a synthesis of the most 
relevant aspects to the decision maker. IBM and other companies that are working in this area should consider the expansion to more applications for the ultimate benefit of the patient.

We can also learn from other regions and their projects. All regions are often confronted with the same problems, and sometimes some regions have found good solutions, or have very useful lessons learned that would avoid unnecessary failures in new projects. Within Europe, Belgium and the Benelux region are a great example for many of the aspects discussed in this thesis: the wide application of MEAs; the standardised development of a yearly unmet medical need list, which uses multi-criteria design analysis to define the diseases that enter the list, and ensures early reimbursement for medicines developed for these diseases (KCE, 2016); the use of health data for decision making and the establishment of public infrastructure for registries, whereby companies are obliged to use and finance the registries within the public system with special rules that apply but remain owners of the data (ISP, 2017). Italy is also a country that can teach a lot on registry generation. Here, the data is owned by the AIFA and the cost of setting the registry is charged to the MAH (Montilla, Xoxi, Russo, Cicchetti, \& Pani, 2015).

\section{Final words}

As discussed in this work, there are many promising projects in Europe that aim to foster timely patient access to innovative and promising new medicines and health technologies. This newly established flexible regulatory approaches incentivise the development of targeted and personalised medicines for reduced patient populations. Very often driven by the regulators and decision makers, they have foreseen the change in drug development towards smaller patient populations following the re-classification of diseases into smaller diseosomes with smaller patient populations.

The main components are already in place, now the pieces of the puzzle have to be refined and make the whole process more integrative and effective by using all available tools in the best possible way and by collaborating with all stakeholders: across borders, across continents, across organizations, across functions and across stages of the drug development process. Many reports describe the changes that need to be implemented, what we need now is action. We should take the examples described above and start implementing the changes and the new tools in order to find ways of steering innovation where it is most needed, facilitating the development of promising medicines, fostering multi-stakeholder collaboration and embracing new processes that will allow timely access to patients who need cures. We cannot forget that patients and citizens are the focus of all these efforts and if they cannot profit from it, it is all in vein. 


\section{References}

ABPI. (2011). Demonstrating Value with Real World Data: A practical guide. London: ABPI.

Brand, H \& Brand A. (March 2017). Verbal communication.

Califf, R. M. (13. Dec 2016). 21st Century Cures Act: Making Progress on Shared Goals for Patients. FDA Voice.

CRA. (November 2016). Managed Entry Agreements in the context of Medicines Adaptive Pathways to Patients. Final report. prepared for EFPIA. London: Charles River Associates.

European Commission. (2001). Directive 2001/83/EC. DIRECTIVE 2001/83/EC OF THE EUROPEAN PARLIAMENT AND OF THE COUNCIL of 6 November 2001 on the Community code relating to medicinal products for human use. Brussels.

European Commission. (September 2016). Strengthening of the EU cooperation on Health Technology Assessment (HTA). http://ec.europa.eu/smart-regulation/roadmaps/docs/2016_sante_144_health_ technology_assessments_en.pdf. Brussels: DG SANTE.

European Medicines Agency. (July 2016). Final report on the adaptive pathways pilot. London: EMA.

European Parliament. (2015). Towards a Harmonised EU Assessment of the Added Therapeutic Value of Medicines. Policy Department A: Economic and Scientific Policy. Brussels: European Union.

EURORDIS, EPF. (13. 05 2015). Call on EU National Competent Authorities for Pricing \& Reimbursement. Brussels: http://www.eurordis.org/sites/default/files/call-on-payers.pdf.

Eggiman, A.-V. (30. March 2017). Industry View - The pillars of decision making: regulators, HTAs, payers / budget holders. DIA Euromeeting 2017, oral presentation. Glasgow.

Eichler, H.-G. (17. February 2017). Podcast: The Evolution of MAPPs.

Eichler, H.-G., Hurts, H., Broich, K., \& Rasi, G. (2016). Drug regulation and pricing - can regulators influence affordability? N Eng J Med, 374, 1807-1809.

Eichler, H.-G., Oye, K., Baird, L., \& et al. (March 2012). adaptive Licensing: Taking the Next Step in the Evolution of Drug approval. Clinical Pharmacology \& Therapeutics, 91 (3), S. 426-437.

EMA and EUnetHTA. (2016). Cooperation between regulators and HTA bodies creates synergies. London: EMA.

EMA. (January 2017). Conditional marketing authorisation. Report on ten years of experience at the European Medicines Agency. Report, European Medicines Agency, London.

EMA Guideline. (25. February 2016). Enhanced early dialogue to facilitate accelerated assessment of PRIority MEdicines (PRIME). EMA/CHMP/57760/2015 . London.

EMA Guideline. (07. 03 2016). GL on the scientific applicantion and the practrical arrangements necessary to implement Regulation (EC) No 507/2006 on the conditional marketing authorization for medicinal products for human use falling within the scope of Regulation (EC) No 726/2004. EMA/CHMP/509951/2006, Rev.1 . London: European Medicines Agency.

EMA. (Nov 2008). Guideline on safety and efficacy follow-up - risk management of advanced therapy medicinal products. EMEA/149995/2008. London: European Medicines Agency.

EMA. (3. July 2007). Orphan drugs and rare diseases at a glance. Abgerufen am 16. February 2015 von www.ema.europa.eu: http://www.ema.europa.eu/docs/en_GB/document_library/Other/2010/01/WC500069805.pdf

FDA. (June 2014). Considering Whether an FDA-Regulated Product Involves the Application of Nanotechnology. Abgerufen am 02. April 2017 von www.fda.gov: https://www.fda.gov/Regulatory Information/Guidances/ucm257698.htm\#_ftn3

Fisher, R., Pusztai, L., \& Swanton, C. (2013). Cancer heterogeneity: implications for targeted therapeutics. British Journal of Cancer, 108, 479-485.

GetReal. (2016). Glossary of Definitions of Common Terms (Including Comments \& Replies from Consultation Rounds). Brussels: IMI.

IMI. (2017). adaptsmart.eu. Abgerufen am 22. 032017 von http://adaptsmart.eu/work-packages/

ISP. (10. 03 2017). Healthdata.be. (I. S. Publique, Produzent) Abgerufen am 22.042017 von https://healthdata.wiv-isp.be/en

Hafen, E., Kossmann, D., \& Brand, A. (2014). Health data cooperatives - citizen empowerment. Methods Inf Med., 53 (2), S. 82-6. 


\section{Chapter 8}

KCE. (June 2016). Future scenarios about drug development and drug pricing. Amsterdam: KCE and Zorginstituut Nederland.

KCE. (20 April 2017). Horizon scanning for pharmaceuticals: proposal for the BeNeLuxA collaboration. Belgian Health Care Knowledge Centre. Brussels: KCE REPORT 283.

KCE. (2016). Multi-criteria decision analysis for the appraisal of medical needs: a pilot study. Belgian Health Care Knowledge Centre. Brussels: KCE .

MoCA-OMP. (2013). Final Report. Process on Corporate Social Responsibility in the Field of Pharmaceuticals Platform on Access to Medicines in Europe Working Group on Mechanism of Coordinated Access to Orphan Medicinal Products (MoCA-OMP).

Montilla, S., Xoxi, E., Russo, P., Cicchetti, A., \& Pani, L. (Jan 2015). Monitoring registries at Italian Medicines Agency: fostering access, guaranteeing sustainability. International Journal of Technology Assessment in Health Care, 31 (4), S. 210-213.

Salmonson, T. (30. March 2017). Early Access Tools: 10 Years' Experience and Lessons Learned with Conditional MAs in EU. Scientific Assessment and Regulatory Decision Making . Glasgow: DIA Euromeeting 2017, oral presentation.

Sanhai, W., Sakamoto, J., Canady, R., \& Ferrari, M. (2008). Seven challenges for nanomedicine. Nature Nanotechnology (3), 242 - 244.

Sherman, R. E., Anderson, S. A., Dal Pan, G. G., Gray, G. W., Gross, T., Hunter, N. L., et al. (8. Dec 2016). Real-World Evidence - What Is It and What Can It Tell Us? The New England Journal of Medicine, 375, S. 2293-2297. 
Curriculum Vitae 

Address

E-mail

EDUCATION

2015-2017 External PhD Candidate at Maastricht University, under the supervision of Prof. Dr. Angela Brand.

2011-2012 Msc International Health Policy (Health Economics) at LSE (London, UK). With major interest in health policy, health economics, product development processes and competition in health care markets. Selected courses were focused on health system models, the pharmaceutical market pricing and reimbursement systems, elaborate concepts of Health Economics, statistical methods in health care economic evaluation, and diverse other aspects such as the social aspects of health care.

$2005-2008$ First Class Honours BSc in Human Genetics at University College of London (UCL). As well as Genetics and Molecular Biology, the degree focused on Pharmacogenomics, Immunology, Virology, Pharmacology and Statistics. The degree project was on HIV nuclear entry pathways. During the degree I developed a strong interest in pharmacogenomics and personalised medicine. University fees financed by the Harold and Olga Fox Scholarship.

2003-2005 Colegio Maravillas "Titulo de Bachillerato" (overall score, 9.72/10); Benalmádena (Málaga, Spain). And the Spanish Baccalaureate: "Selectividad" (overall score, 8,91/10)

\section{WORK EXPERIENCE}

Jul 16 - Clinical Study Reviewer and inspector at Swissmedic, Bern, Switzerland (for a description see below).

Oct 14-Apr 16 Scientific administrator, European Medicines Agency (EMA), London, UK. National Expert on Secondment at the EMA in the clinical pharmacology scientific support office. I am responsible for the peer review of clinical pharmacology aspects in the MAA dossier of centrally approved pharmaceuticals. As well as, for the secretariat of the Pharmacokinetics Working Party, for providing assistance in answering questions on clinical pharmacology 
Jan 13 - Oct 14 GCP Inspector and Clinical Study Reviewer at Swissmedic, Bern, Switzerland. The Swiss therapeutic agency, within other activities, is in charge of evaluating and approving clinical trials (in relation to the safety and quality aspects) and carrying out GCP and Pharmacovigilance inspections of clinical trials (CT). In my position at the agency I performed both described tasks and developed an extensive knowledge in regulatory processes. The evaluation of CT applications is based on Swiss and international applicable legislation and guidelines. ICH guidelines and EMA position papers are strongly taken into consideration in every day's work. For the safety aspect, information about the preclinical battery of tests (according to ICH M3 within others) and the available clinical data, including aspects on pharmacology, pharmacogenomics and drug interactions, was reviewed critically. Other important responsibilities were the participation in the Swissmedic Nanomedicine Task Force, the contact with international regulatory agencies, the representation of the agency at national and international conferences with presentations, giving courses on Clinical Trials in Switzerland, responding to questions from the public and the adaption of current documents, SOPs, websites, forms and guidelines to the Swiss legislation that came into force on 01.01.2014.

Jun - Aug 12 Placement at the Department of Health Strategy Unit (London, UK). Working along the strategy advisors, my role was to support their work for the ministry. Furthermore I studied the prevention programs for CVD diseases in PAHO countries, their effects and how they could be applied in the UK.

Jan-Mar 12 Research assistant for Policy Cures (part-time during MSc. program). Involved in a project that evaluated differences in treatment strategies across Europe in Rheumatoid Arthritis. Review of guidelines, regulations and interviews with physicians. Responsible for France and Belgium.

Jan 09-Apr 11 Clinical Research Associate at Geistlich Pharma AG (Switzerland). Involved in the planning, investigator selection, monitoring and reviewing of clinical trials -including on-site visits-. Extensive work under ICH-GCP guidelines. Also worked on several innovative projects (pull and push) to bring new 
products into the market. Furthermore, I represented the clinical research department in many project teams and represented the company in several international conferences. Continuous contact with external partners such as Key Opinion Leaders and other internal departments was part of my daily work.

Jun - Aug 07 Internship at the Swiss National Centre for Retroviruses (NZR, Zurich). The project focused on a special and unusual HIV patient.

\section{LANGUAGES AND OTHER SKILLS}

- Spanish (native): Fluent in speaking, reading and writing

- English and French: Fluent in speaking, reading and writing

- German: Fluent in speaking and reading, advanced in writing

- Italian and Croatian: Basic knowledge in speaking and reading

- Microsoft Package, STATA and Treeage: Very good knowledge. 



\section{Valorisation}



Although personalised medicine has not yet fulfilled its expectations, the advances in biology and genetics will lead to an increasing number of personalised medicines in the future. The analysis performed in chapters 2 and 3 will help with adapting the current environment in order to facilitate the implementation of personalised treatments. It is important to identify the aspects that need to be adapted and the areas of healthcare that need to change for its implementation. The literacy aspect discussed in chapter 2, 3 and 8, and the proposal to adapt the current curricula is paramount for the future and represent an immediate need. Universities should already be considering this. The newly created academic office at the EMA can help with this aspect and influence universities to change their curricula.

The research performed under the Coordination \& Support Action (CSA) PerMed has already been implemented into a Strategic Research and Innovation Agenda (SRIA) that has guided the creation of the International Consortium for Personalised Medicine (IC PerMed) and its action plan recently published under the title "Actionable research and support activities identified by the International Consortium for Personalised Medicine" (March 2017). IC PerMed had it's first workshop with experts and funders in June 2017 in Milan. The SWOT and gap and needs analysis presented in chapters 2 and 3 are helping national funding agencies to identify the most urgent areas of research for the implementation of personalized medicine and guide their research funding activities.

It is clear from today's perspective, that the question formulated in chapter 4 and the main conclusions drawn were and are really relevant: since 2015, when the article was published, all stakeholders have gained interest in conditional approvals. This is evident from the adaptive pathways pilot, the IMI project AdaptSmart and the agendas from the STAMP ${ }^{23}$ meetings, between others. The EMA 10 year report on conditional marketing authorisations even states "High interest in the conditional marketing authorisations and its relevance in the context of timely access to medicines have also led to it being discussed in various fora, including the European Commission Expert Group (...) ("STAMP"), and being referred to in conclusions of the Council of the European Union on strengthening the balance in the pharmaceutical systems in the EU and its Member States ${ }^{5}$ " (EMA, January 2017). What is left now is to encourage the right use of these promising tools that aim for timely patient access to promising medicines; and to enter discussions with "down-stream" stakeholders to seek their acceptance of conditional approvals. Furthermore, the tool box for evidence generation needs to be developed, including the use of RWD so that it fits to the requirements and expectations of all stakeholders and decision makers, as discussed in chapter 8. Chapters 2 and 3 claim new regulatory tools are needed, however, after evaluating the available

\footnotetext{
${ }^{23}$ European Commission Expert Group on Safe and Timely Access to Medicines for Patients
} 
tools in Chapter 4, we reject this conclusion and claim that the tools are already available, they just have to be used correctly.

Stakeholders are also becoming interested in Managed Entry Agreements (MEAs). The future application of this $\mathrm{P} \& \mathrm{R}$ tool is very promising; however, there are some technical aspects that need to be improved in order to facilitate and extend their use. Methods based on data analytics, Electronic Health Records (EHRs) and Artificial Intelligence (AI) need to be developed in order to reduce the resources needed to implement them both on the side of the payer and the developer. Once these methods are available, game theory, as discussed in chapter 7, could be used to model the different scenarios and find the most appropriate model that maximises the utilities of all stakeholders; having in mind not only the effect on price, profit and innovation, but considering the bigger picture of net impact on welfare of this and future generations (Sood, De Vries, Gutierrez, Lakdawalla, \& Goldman, 2009). MEAs could help to apply adaptive pricing and adaptive value proposition that would fit perfectly with the adaptive licensing proposals such as conditional approvals.

Chapter 6 lays all the possibilities for the application of big data in health care and drug development. The advances in this area will bring huge improvements into the healthcare market, if applied correctly. Patients should become more empowered to gather their data and create patient-owned data collections in order to ensure that the ethical concerns in health-data sampling and storing are addressed correctly and to the benefit of the patient. As already done in Belgium and Italy, the government could engage in creating the infrastructure for these type of registries. Going further than they already do, this infrastructure could be given to the patient organizations in order to create patient-centred registries that are owned by the patients and can be sold to the developers who need it.

Following the conclusion of chapter 7 , one of the big applications of this work is to apply game theory to all stages of the drug development process, where rationale decision-making is needed. Going over the needs of drug development, game theory could also be applied broadly in healthcare. Experts in game theory can use the presented framework to create models and games that help companies and decision makers to maximise their utilities and improve the whole healthcare sector.

It is clear to the author that this thesis is very descriptive and what is needed now is to find useful applications to the topics described. But sufficient ideas and proposals have been framed to start with small steps that lead in the future to global structural changes. Only like this, patients and citizens will be able to enjoy the full promises of personalised medicine and flexible regulatory tools. 
Table 0-1. List of publications and citations (up to 24.07.2017). Grey shadowed articles are part of the $\mathrm{PhD}$ thesis.

\begin{tabular}{|c|c|}
\hline Article & Citations \\
\hline $\begin{array}{l}\text { Leyens L, Reumann M, Malats N, Brand A. Use of big data for drug development and for } \\
\text { public and personal health and care. Genet Epidemiol. } 2017 \text { Jan;41(1):51-60. doi: } \\
\text { 10.1002/gepi.22012. Epub } 2016 \text { Nov 21. PubMed PMID: } 27873357 .\end{array}$ & 1 \\
\hline $\begin{array}{l}\text { Vozikis A, Cooper DN, Mitropoulou C, Kambouris ME, Brand A, Dolzan V, Fortina P, Innocenti } \\
\text { F, Lee MT, Leyens L, Macek M Jr, Al-Mulla F, Prainsack B, Squassina A, Taruscio D, van Schaik } \\
\text { RH, Vayena E, Williams MS, Patrinos GP. Test Pricing and Reimbursement in Genomic } \\
\text { Medicine: Towards a General Strategy. Public Health Genomics. 2016;19(6):352-363. doi: } \\
\text { 10.1159/000449152. Epub 2016 Sep 28. PubMed PMID: } 27676083 \text {. }\end{array}$ & 4 \\
\hline
\end{tabular}

Leyens L, Brand A. Early Patient Access to Medicines: Health Technology Assessment Bodies 4 Need to Catch Up with New Marketing Authorization Methods. Public Health Genomics. 2016;19(3):187-91. doi: 10.1159/000446537. Epub 2016 Jun 1. PubMed PMID: 27238553; PubMed Central PMCID: PMC5296921.

Negrouk A, Horgan D, Gorini A, Cutica I, Leyens L, Schee genannt Halfmann S, Pravettoni G. 2 Clinical Trials, Data Protection and Patient Empowerment in the Era of the New EU Regulations. Public Health Genomics. 2015;18(6):386-95. doi: 10.1159/000441561. Epub 2015 Nov 14. PubMed PMID: 26565798.

Syme R, Carleton B, Leyens L, Richer E. Integrating Personalized Medicine in the Canadian Environment: Efforts Facilitating Oncology Clinical Research. Public Health Genomics. 2015;18(6):372-80. doi: 10.1159/000441560. Epub 2015 Nov 14. PubMed PMID: 26565702.

Leyens L, Richer É, Melien Ø, Ballensiefen W, Brand A. Available Tools to Facilitate Early 5 Patient Access to Medicines in the EU and the USA: Analysis of Conditional Approvals and the Implications for Personalized Medicine. Public Health Genomics. 2015;18(5):249-59. doi: 10.1159/000437137. Epub 2015 Aug 28. PubMed PMID: 26316202.

Horgan D, Jansen M, Leyens L, Lal JA, Sudbrak R, Hackenitz E, Bußhoff U, Ballensiefen W, Brand A. An index of barriers for the implementation of personalised medicine and pharmacogenomics in Europe. Public Health Genomics. 2014;17(5-6):287-98. doi: 10.1159/000368034. Epub 2014 Nov 15. PubMed PMID: 25401385.

Leyens L, Horgan D, Lal JA, Steinhausen K, Satyamoorthy K, Brand A. Working towards personalization in medicine: Main obstacles to reaching this vision from today's perspective. Personalized Medicine. 2014; 11(7):641-649 - November 2014. DOI: 10.2217/PME.14.55

Zaitseva L, Cherepanov P, Leyens L, Wilson SJ, Rasaiyaah J, Fassati A. HIV-1 exploits importin 52 7 to maximize nuclear import of its DNA genome. Retrovirology. 2009 Feb 4;6:11. doi: 10.1186/1742-4690-6-11. PubMed PMID: 19193229; PubMed Central PMCID: PMC2660290. 

Summary 



\section{Short summary of the chapters:}

Chapters 1-3 set the scene for the development of personalised treatments and analyse the gaps and needs for its wider implementation, as well as the current barriers. In chapter 4 we describe the available regulatory tools that aim to facilitate earlier patient access to promising medicines, showing that these tools are already available but not optimally used. Chapter 5 outlines the need to have flexible approaches at the HTA level in order to guarantee early patient access through flexible MAA processes. The last two chapters, focus on tools that can be used for decision making: in chapter 6, we discuss big data from the perspective of drug development and public health laying out the needs to ensure that it fulfils its promises for healthcare; whereas in chapter 7, we propose a framework to apply game theory to drug development.

\section{Summary of the topic of the thesis:}

Rapid advances in 'omics' sciences and technologies have facilitated the development of personalized medicine. However, there are some areas that need revision in order to reach the full implementation of personalised medicines. These gaps and needs are described in detail in the thesis, one of the most important aspects is the need for multi-stakeholder dialogue and collaboration across scientific fields and across regions. One of the aspects that have been evaluated more in detail is how the current drug development process can address the approval of personalised medicines with approaches that are more flexible than the standard drug approval process. The innovative methods developed by national and international medicines agencies to expedite promising new medicines to the market and facilitate early patient access, such as the conditional approval and the adaptive pathways, are described. We conclude that the flexible tools are available but the way we use them has to improve. However, the efforts from medicines agencies are welcome but will be in vain if health technology assessments do not follow with similar adaptive and flexible initiatives, and the European 'postcode' lottery will continue. One decision-supporting tool that can be used by decision makers is big data. Data analytics can be used across the entire healthcare value chain, from drug discovery and development through epidemiology to informed clinical decision for patients or policy making for public health. Big data has the potential to improve general health outcomes if we learn how to exploit it in the right way. Game theory is another decision supporting tool that can be applied for rational decision-making. In this work we describe a framework for its application in drug development and we give an example of how it could be applied. This application has an enormous unravelled potential. Finally, a framework is proposed, which explicitly puts together all tools that are available 
to increase the flexibility of the drug regulatory system and makes it adaptive to the best available evidence. Tools such as early multi-stakeholder dialogue, iterative dialogue, implementation of control mechanisms, successive rounds of HTA with the new evidence and the introduction of MEAs and adaptive pricing mechanisms are part of the framework. 


\section{Acknowledgements}



Dear Angela, I do not know how to thank you enough for the amazing journey you have taken me on. You gave me access to interesting projects, incredible opportunities and the most important part: I have met extraordinary people who have enriched me enormously. You are an inspiration, a visionary and a beautiful person who always gets the best out of people. Whoever does not see this is blinded by their wish to be a little bit more like you. You gave me wings to fly and I promise I will use them to go as high as I can to make a difference someday.

Dear Helmut and dear Nuria, thank you for also accompanying me on this journey and helping me so much with your kind advice, great discussions and infinite warmth.

I would also like to thank my parents. They are brilliant, extremely intelligent and kind and they have enlightened my way since I was born. They have sacrificed so many of their wishes for me and they knew how to give me the right set of tools to become independent and know how to react to all faces of reality: the bright ones and the not so bright ones. Being a parent myself, I know how difficult this is to achieve. They could not have done it better. Their persistent encouragement, education and guidance through my childhood and teenage years have made me the confident and strong-willed person I am today. I hope I can someday give you back all you have done for me. THANK YOU. A special mention goes to my great husband Juan Fernando. I am the luckiest person in the World to have found somebody who understands me so well, who has the same interests as me and who supports me in every step of every project I start. He has endured the hard moments during the preparation of the $\mathrm{PhD}$ and always knows how to reduce my stress, give me strength and show me how to enjoy every second of life. Thank you for your unconditional love and patience! And our daughter Sofía, well what can I say, she is the greatest child in the World, so curious, happy, always with new ideas of what to do and so easily amazed by everything in nature. She reminds me every day to enjoy every single moment of life and enlightens my days with her laughs.

I would also like to mention the people that have guided me from childhood to be where I am, giving me great advice and motivation. Thank you to Jeronimo Hall, my athetlics trainer, he teached me perseverance, effort and enduring pain to reach difficult goals. D. Miguel Conesa, my biology teacher, who I have to thank for my love in genetics and Pepa Cejas, my English teacher who encouraged me to pursue my crazy dream of doing my bachelors in the UK and wrote the most amazing recommendation letter one could imagine. At UCL I was very inspired by Steve Jones, Ariberto Fassati my thesis professor and other great professors, but most importantly my degree colleagues, who helped me to actually get through that first year when English was an enormous challenge: Andy, Natalia, Rachit, Amanthi: thank you for always being there for me and helping me to believe in myself. Also, thank you to David Gems who advised me to not do a PhD straight after the BSc. and get some work experience in order to find the topic that I am passionate about. 
Christoph Goerlach, thank you for believing in me when I was just 21 and offering me my first job in the real World. The experience you gave me has been so valuable ever since! Ela it was such a gift to meet you, you have been a great friend and mentor, thank you. Gaby, Natalia and Toby, thank you for making me feel at home in Switzerland. Françoise Jaquet, I am so grateful you gave me the opportunity to work at Swissmedic, you are a great boss and an amazing person. I would also like to thank Marisa Papaluca and Falk Ehmann to select me to join them at the EMA and to guide me through my first year with great advice, and to thank Ana HidalgoSimons to understand so well my personal circumstances and despite them continue being a great reference. Jorge Martinalbo, you have made my mind work harder than anyone before in our intense and interesting discussions. I hope you are once able to see how genius you are. And my 'PhD brother' Matthias Reumann, thank you for the inspiring article we wrote together in perfect harmony and the enriching discussions we have had since!

During the PhD I have met incredible people who I cannot leave unmentioned, Etienne Richter, Erica Hackenitz, Wolfgang Ballensiefen, Øyvin Melien, and so many others: Thank you all for your input, advice and motivation. 


\section{Annex 1}

\section{CSA PerMed: Europe's commitment to Personalised Medicine}

Published as: Leyens L., Hackenitz E., Horgan D., Richer E., Brand E., Bußhoff U. and Ballensiefen W. on behalf of the PerMed consortium (2014). CSA PerMed: Europe's commitment to Personalised Medicine. Eurohealth incorporating Euro Observer. 2014: Vol.20, No.3. 41-44. 



\section{Summary}

Personalised medicine is one of the most innovative areas in the future of health research. At present, its full potential cannot be developed due to fragmented activities, insufficient communication, and lack of generic solutions in the different areas of personalised medicine; moreover, implementation is a major challenge. The EU-funded Coordination \& Support Action PerMed was initiated to step up coordination efforts between key European stakeholders, to allow synergies and avoid duplication or competition, and to provide recommendations to foster the implementation of personalised medicine in transnational research and health systems.

\section{Personalised Medicine: present and future}

Health care as we know it is radically changing to give way to increasingly more personalised health interventions for citizens and offering more personalised therapies and treatments for patients. Essentially, Personalised Medicine (PM) is an innovative method of treating citizens and patients that utilises research, data and up-to-the-minute technology to provide better diagnostics and follow-up for citizens than is currently the case. Among others, it uses genomic information to discern whether a particular intervention will work for a particular patient and assists clinicians in deciding which treatment will be the most effective. It can also have a huge impact in a preventive sense (see Box 1).

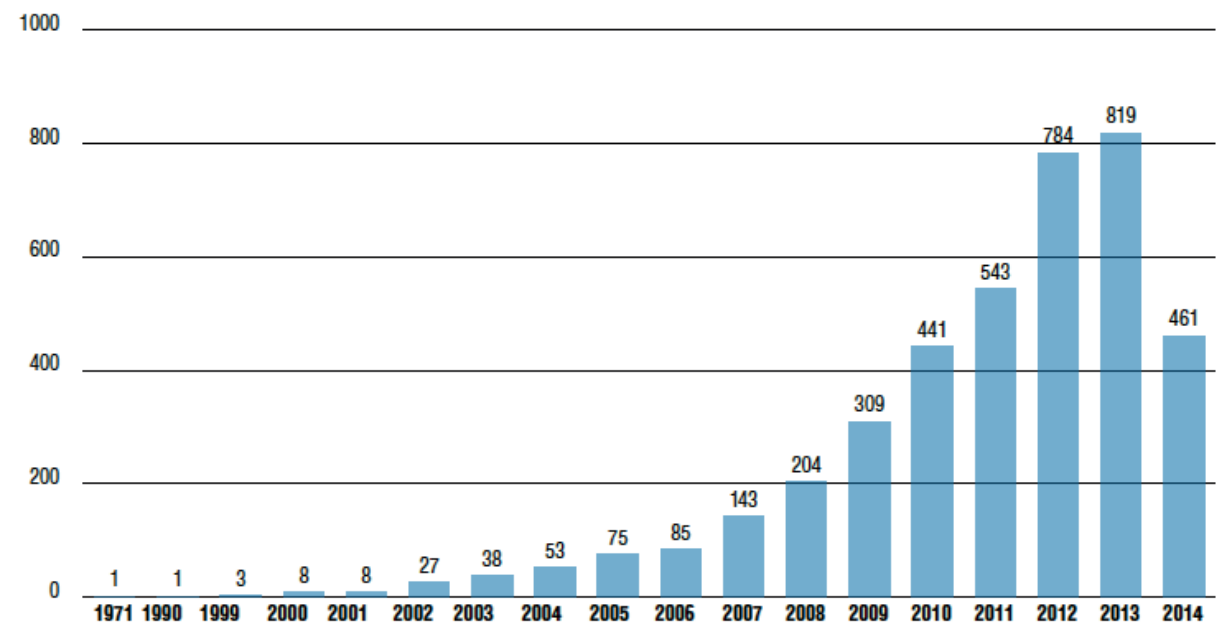

Figure 1: Number of publications per year on Personalised Medicine, 1971 - 2014. Source: PubMed, last search on 1 June 2014 with search terms "personalized medicine"- or "personalised medicine" in title or abstract 
However, we are only at the beginning of the road and many challenges have to be overcome in order to benefit from PM's full potential. The era of 'one size fits all' in medicine is slowly coming to an end. Personalised treatment options are being developed for an array of conditions and some have already entered the market. Treatments for cancer are leading the field, but they are followed closely by treatments in cardiovascular, pulmonary, infectious and psychiatric conditions, among others. Personalised therapies aim to provide "the right treatment to the right patient at the right time", with early diagnosis, increasing efficacy, decrease in adverse drug reactions, and cost-effective treatments that may result in cost savings, quality of life improvement, and reduction of general morbidity in the population.

Box 1: Definition of Personalised Medicine. Source: Ref. 1

"Personalised medicine refers to a medical model using molecular profiling for tailoring the right therapeutic strategy for the right person at the right time, and/or to determine the predisposition to disease and/or to deliver timely and targeted prevention."

Despite being a concept already applied by Hippocrates more than two thousand years ago in Ancient Greece, the advances in the so-called "-omic" sciences (genomics, transcriptomics, proteomics, metabolomics, etc.) and in Information and Communication Technologies (ICT) have led to enormous advances in the field of PM in the past two decades. Greater understanding of the molecular basis of disease and all the factors, such as environmental factors influencing disease onset, progression and response to treatment, together with the staggering fall in the costs of gene or genome sequencing and genotyping, plus faster results availability, have resulted in the market entry of over twenty personalised therapeutics; such as Herceptin, the first personalised treatment approved sixteen years ago for HER2+ metastatic breast cancer. The development of many more is underway. However, the scope and approach of PM is not limited to the treatment of diseases only; it is much broader and inclusive, also covering areas such as lifestyle advice, prevention, environmental interventions and even the structure and organisation of hospitals and health systems. Furthermore, the increased interest from physicians, decision makers, regulators and the general public in PM has contributed to its increased application. Figure $\mathbf{1}$ shows the number of publications on PM in the past 40 years, and their exponential rise particularly in the last ten years. Notwithstanding the great interest, we are still only at the start.

Although the US may have been leading the field in the past, Europe is showing a clear commitment to PM. Reports have been published by the European Commission (EC) supporting the mission of personalisation of health and many politicians and decision makers have expressed their support. For example, in the 
UK, Germany and France, the national governments have made a strong commitment - implicitly or explicitly - to genomic medicine and the application of PM, mostly in cancer research and treatment. Furthermore, the EC's new Horizon2020 research grants programme - initiated in January 2014 - will promote research in all aspects of targeted therapies, including ICT to assist decision making in PM. As the Director General of DG Health and Consumers (DG SANCO), Paola Testori Coggi, puts it "it is essential for Europe to build on our strengths to develop innovations to promote growth and benefit European citizens. Genomics has the potential to be a key sector contributing to this in the future ... Advances in PM can bring business development and economic growth to Europe in addition to improved prevention, treatment and care to European citizens." These objectives form the basis of CSA PerMed, otherwise known as the Coordination \& Support Action (CSA) Personalised Medicine 2020 and beyond (see Box 2).

The development of PM in Europe represents an important paradigm shift for all health care systems and poses major challenges - both for the present and the future. These challenges need to be overcome to meet the objectives of Europe 2020, the Digital Agenda, the Innovation Union and Horizon 2020 by bringing together: research for health, innovation for health and health equity, and significant contributions to global research and innovation systems. A general change in mind-set in health care delivery is also needed.

\section{Realising potential benefits}

Why should we strive towards the personalisation of health care and promote the four Ps in health (predictive and preventive, personalised and participatory)?

There are potential benefits from applying evidence-based personalised treatments, including:

- improvement of informed medical decisions

- shift from reaction to disease towards prevention and prediction of disease

- targeted therapies with higher probability of success

- risk reduction with fewer adverse reactions to medicines

- timely/early disease interventions

- cost-efficient treatment solutions and general health care cost containment. 
Box 2: The PerMed consortium

CSA PerMed is a consortium - created by decision makers in Europe, including more than ten ministries and funding bodies - which aims to prepare Europe to be a global leader in the implementation of PM. It differs from other consortia and working groups due to the partners involved and its aim to carry out focussed discussions on concrete research actions, rather than prolonging on-going broad discussions and recommendations (see www.permed2020.eu). Moreover, transparency, openness, collaboration and the avoidance of duplication lie at the core of the CSA PerMed approach. The consortium's unique features create the potential to develop a strategic research and innovation agenda for Europe (SRIA) and be the starting point for a European Innovation Partnership (EIP) in PM acting across the entire research and innovation chain, bringing together key actors at European, national and regional level.

For health systems as a whole, potential benefits include early systematic dialogue between the relevant key stakeholders, citizen-centred health care systems, encouragement of patients to be more active in their health management and feel greater ownership in the responsibility of their health, support quality of life, health and wellness, yield a maximum return on health care investment and adjustment to the needs of sub-sectors of the population, among others.

Nevertheless, a great deal still needs to be done to reach these benefits across the entire health care spectrum, and not restrict them to a limited number of conditions. The challenges have been widely discussed and described in a large number of reports and publications (see next paragraph for examples).

\section{The way forward}

PerMed has identified and evaluated the information already available as well as the strategy documents published by key stakeholders, including reports, guidelines and roadmaps on PM. A gaps and needs analysis was performed on 18 relevant reports - from the EC, the European Science Foundation (ESF), the European Alliance for Personalised Medicine (EAPM), the Public Health Genomics European Network (PHGEN), the European Medicines Agency (EMA), the iNNOVAHEALTH Conference under the Cyprus EU Presidency and the European Hospital and Healthcare Federation (HOPE) among others - and over 35 interviews were carried out with relevant stakeholders.

Regardless of their authors, interests and target group, these reports and interviews reach similar conclusions on the aspects that need to be tackled. These are: 


\section{Targeted research in molecular mechanisms and ICT}

Targeted research to better understand the molecular mechanisms of disease and all implicated factors, as well as the identification and validation of biomarkers, is essential for the development of further personalised therapeutics. Multidisciplinary research teams, joining the knowledge from a variety of sciences, together with cross-disciplinary and cross-border collaboration in research and in drug development are essential parts of the R\&D process of PM. Further developments in data collection, storage, management, sharing, mining, processing and analysis are also imperative. ICTs have not been exploited to their full potential and will surely push forward the individualisation of medicine in all areas (research, translation, diagnosis, treatment decision making, follow-up, etc.).

\section{Adaptive business models, translational pathways and systematic early dialogue}

The current business model for pharmaceutical companies is no longer valid once we move away from the "one size fits all" drugs. Pre-competitive collaboration between companies (pharmaceutical companies and medical device manufacturers, for example), the increase in public-private partnerships and a more flexible and adaptive business model is needed for the development and translation into health care of personalised technologies. Furthermore, systematic early dialogue with regulators and patients at an early phase of development would lead to more efficient drug development and translation processes. Clinical trial designs need to change: Phase III studies with thousands of patients are not possible and adaptive designs with smaller numbers of patients are needed, like the ones already being conducted in cancer that permit the application of personalised treatment options under one protocol. New dynamic and sustainable pathways that lead to timely and effective translation of innovative technologies into health policies and health care are needed, always ensuring high quality, safe and efficient treatments entering the market.

\section{Make regulation simple, coherent and predictable}

In addition, the regulations that are in place nowadays do not consider the specificities of personalised interventions, including therapeutics. Many of the ones that affect PM are being revised, but remain far from ideal. Especially in Europe considering the inherited heterogeneity of our Member States - simplified, harmonised, coherent (across directives and regulations) and predictable regulatory procedures are welcomed. Some positive steps forward are the new medical devices directive regulating (for the first time in Europe) in-vitro and companion diagnostics, and the proposed adaptive licensing model from EMA. In 
order to expand its leadership role, it is PerMed's view that Europe could engage in international efforts to harmonise regulatory aspects.

\section{Driving health care systems towards preventive care}

When it comes to health care systems, a general change in mind-set in health care delivery and provision is needed. From a coordinated reimbursement process for drugs and diagnostics, new financing strategies, new structures and models at the provider level, updated health care professional training and a change in attitudes, a shift towards preventive care, towards new cost assumption models, changes in patient behaviour and an increased interest and literacy from citizens in general are needed. The social consequences of the implementation of PM have not been fully studied, and there are many ethical challenges that lay ahead, which is why the principles of "Ethical, Legal and Social Implications" (ELSI) are essential and need to be further explored by research and applied by all stakeholders.

\section{Conclusion}

Even though PM may be one of the most innovative areas in the future of health research, the full potential for patients, citizens and the economy in Europe currently cannot be realised due to the inherited fragmentation between European Member States, inadequate communication and lack of common vision on the solutions that are needed. Appropriate governance levels are required to solve these challenges.

PerMed aims to provide concrete recommendations and to take a big step forward towards PM for all, without forgetting that the ultimate goal is to bring the right health intervention to the right patient at the right time, to avoid as many adverse reactions as possible during treatment, to make it affordable for health care systems and to ensure equality in access to personalised innovations. As long as the interests of citizens drive work towards this common mission, Europe can become a leader in PM, with the potential to also create business and economic growth and, most importantly, give patients access to safe, highly efficient and targeted treatments in a timely and cost-efficient manner. 


\section{References}

1. European Commission. Use of '-omics' technologies in the development of personalised medicine. Brussels: European Commission, 2013.

2. Technology Strategy Board. Stratified Medicines in the UK, Vission and Roadmap. Swindon: Technology Strategy Board, October 2011.

3. BMBF. Personalised Medicine - Action Plan, a new approach in research and health care. Germany: Federal Ministry of Education and Research (BMBF), February 2013.

4. Claeys A, Vialatte J-S. Advances in genetics: towards a Precision Medicine? Technological, social and ethical scientific issues of personalised medicine. [Les progrès de la génétique : vers une médecine de précision ? Les enjeux scientifiques, technologiques, sociaux et éthiques de la médecine personnalisée], January 2014. Available at: http:// www.ladocumentationfrancaise.fr/ rapports-publics/144000117/index.shtml

5. Testori Coggi P. A European view on the future of personalised medicine in the EU. Eur J Public Health 2011;21(1):6 - 7.doi: 10.1093/eurpub/ckq202

6. European Commission. Europe 2020, the Digital Agenda, the Innovation Union and Horizon 2020. Available at: http://ec.europa.eu

7. European Science Foundation. Forward Looks. Personalized Medicine for the European citizen. Towards more precise medicine for the diagnosis, treatment and prevention of disease (iPM), 2012.

8. EAPM. Innovation and Patient Access to Personalized Medicine, 2013. Available at: http:// euapm.eu/wp-content/uploads/2012/07/ EAPM-REPORT-on-Innovation-and-Patient-Access-toPersonalised-Medicine.pdf

9. Brand A, Lal JA. European Best Practice Guidelines for Quality Assurance, Provision and use of Genome-based Information Technologies: the 2012 Decalaration of Rome. Drug Metabolism and Drug Interaction Studies 2012; $27(3): 177$ - 82.

10. European Medicines Agency. Pilot project on Adaptive Licensing. Available at: http://www. ema.europa.eu/docs/en_GB/document_library/ Other/2014/03/WC500163409.pdf

11. iNNOVAHEALTH. Building on Open Innovation ecosystem in Europe for Healthcare, October 2012. Available at: http://www.enterpriseisrael.org.il/ wp-content/uploads/2013/04/InnovahealthReport.pdf

12. HOPE and PwC. Personalized Medicine in European Hospitals, 2011. Available at: http:// www.hope.be/05eventsandpublications/ docpublications/88_personalised_medicine/88_ HOPEPWC_Publication-Personalised-Medicine_February_2012.pdf 


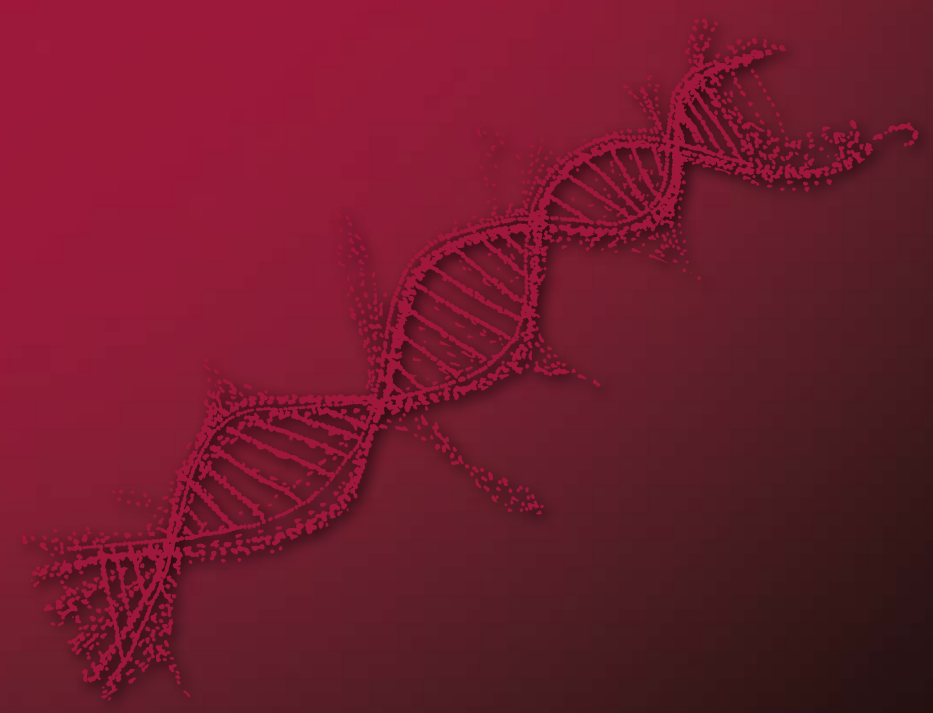

